ESAIM: M2AN 49 (2015) 1085-1126

DOI: $10.1051 / \mathrm{m} 2 \mathrm{an} / 2015001$
ESAIM: Mathematical Modelling and Numerical Analysis

www.esaim-m2an.org

\title{
ANALYSIS OF A HIGH-ORDER SPACE AND TIME DISCONTINUOUS GALERKIN METHOD FOR ELASTODYNAMIC EQUATIONS. APPLICATION TO 3D WAVE PROPAGATION
}

\author{
Sarah Delcourte ${ }^{1,2}$ And Nathalie Glinsky ${ }^{3,4}$
}

\begin{abstract}
In this paper, we introduce a high-order discontinuous Galerkin method, based on centered fluxes and a family of high-order leap-frog time schemes, for the solution of the 3D elastodynamic equations written in velocity-stress formulation. We prove that this explicit scheme is stable under a CFL type condition obtained from a discrete energy which is preserved in domains with free surface or decreasing in domains with absorbing boundary conditions. Moreover, we study the convergence of the method for both the semi-discrete and the fully discrete schemes, and we illustrate the convergence results by the propagation of an eigenmode. We also propose a series of absorbing conditions which allow improving the convergence of the global scheme. Finally, several numerical applications of wave propagation, using a $3 \mathrm{D}$ solver, help illustrating the various properties of the method.
\end{abstract}

Mathematics Subject Classification. 35L50, 35F10, 35F15, 35L05, 35Q99.

Received July 11, 2012. Revised October 17, 2014.

Published online June 30, 2015.

\section{INTRODUCTION}

In the last few decades, the computational seismology has become an essential tool to simulate realistic wavefields of local, regional or even global wave propagation problems. The physics governing these phenomena is now well-understood and many different accurate numerical methods have been developed and can deal with three-dimensional realistic applications thanks to a continuous increase of the computational resources and the use of parallel computational facilities.

Among all the numerical methods proposed for simulations in time domain, the most popular is undoubtedly the finite difference (FD) method and its many improvements from the initial FD schemes proposed by Alterman and Karal [7] or Kelly et al. [41] such as, for instance, the introduction of the velocity-stress system and the staggered-grids $[47,61]$, fourth-order schemes in space $[9,45]$ and rotated staggered-grids [57] allowing strong fluctuations of the elastic parameters. If these techniques lead to simple and cheap algorithms, their

Keywords and phrases. Discontinuous Galerkin method, centered flux, leap-frog scheme, elastodynamic equation.

1 Université de Lyon, CNRS UMR5208, Université Lyon 1, Institut Camille Jordan, 43 blvd du 11 novembre 1918,69622

Villeurbanne cedex, France. delcourte@math.univ-lyon1.fr

2 UCBL/INRIA Grenoble Rhône-Alpes/INSMI - KALIFFE, France.

3 IFSTTAR/CEREMA, DTer Méd., 56 boulevard Stalingrad, 06359 Nice cedex 4, France.

4 INRIA Sophia Antipolis Méditerranée, team Nachos, 2004 route des Lucioles, 06902 Sophia Antipolis cedex, France. 
major drawback is the restriction to cartesian grids not suited for geometrical internal or surfacic nonlinearities (topography) but, the use of non uniform grids [54] or even discontinuous grids [6] enabled improving the accuracy at the free surface and, more recently, an hybrid method coupling a finite-difference technique for the most part of the domain and a finite-element method in subdomains containing the nonlinearities (topography, faults) has been proposed [33,51]. Some other methods have been further developed such as finite element (FE) methods which allow meshes adapted to complex geometries $[8,46,48]$. However, they are often very costly because one needs to invert a global mass matrix at each time step which is not well adapted to the use of high-order space approximations. This difficulty was overcome by the use of Gauss-Lobato Legendre quadrature formulae at the root of the spectral element methods (SEM), usually applied to second-order system in displacement (see, for instance $[14,42]$ amongst many contributions). However, except some applications to triangular meshes $[49,50]$ and resulting in non-diagonal mass matrices, these methods are mostly developped for quadrangular or hexahedral discretizations which may complicate the construction of meshes adapted to heterogeneous media. As an attractive alternative for a better representation of complex geometries, non conforming discontinuous Galerkin spectral element methods have been recently developped and analysed for the wave propagation in 2D quadrangular meshes [5] or 3D affine hexahedral meshes using a velocity-strain formulation $[13,63]$ for coupled elastic/acoustic media. When methods based on conforming meshes are used, simplicial meshes permits a better approximation of complex geometries (topography, faults). For this reason, we study a high-order Discontinuous Galerkin (DG) method applied to tetrahedral meshes.

The Discontinuous Galerkin (DG) method has been initially introduced by Reed and Hill [55] for the solution of neutron transport problems. A first analysis of the method for hyperbolic equations has been presented by Lesaint and Raviart [44]. Their result has been improved and broadened by Johnson et al. [36,37]. The method is now very popular for solving many problems of physics governed by hyperbolic partial differential equations. Detailed reviews of methods and applications can be found in Cockburn et al. [22] or Hesthaven and Warburton [31].

This method can be seen as a discontinuous finite element method with a great flexibility in the choice of the local degree $p$ of the polynomial interpolation while being adapted to most meshes such as unstructured or non-conforming discretizations. Both aspects can be combined to build high-order hp-adaptive finite element methods [24,59]. The major advantage of this method is an easy extension to high-order in space realized by local basis functions defined in each element. The type of basis functions is not fixed and many families are available with their own advantages/drawbacks that can impact the accuracy [23]. The inversion of a global mass matrix is avoided when an explicit scheme in time is used since all calculations are done at the local level producing block diagonal matrices. On the contrary, fluxes between neighboring elements must be accounted for and many strategies have been studied in the literature (for instance, see [31]). More, it has a simple computational pattern with elements which makes the method naturally suited for parallelization.

Among the pionering methods, Cockburn and Shu [18] introduced a class of high-order discontinuous Galerkin finite element methods, based on upwind fluxes and a Runge-Kutta time scheme, for 1D scalar conservation laws. The method was then extended to systems and multidimensional cases in [19-21] and the analysis proved the good convergence properties of the method. Other properties are also important to define an accurate scheme. The study of the dispersive and dissipative properties of a method provides information on its ability to accurately propagate a wave, especially high frequency, along many wavelengths (as it is the case for simulation of realistic problems). Hu et al. [32] studied dispersive and dissipative properties of a DG method for both 1D and 2D equations. They concluded that they depend on the type of fluxes: the error being dominated by dissipation for upwind fluxes whereas the dissipation rate is zero for centered fluxes. Sherwin [58] compared the dispersive properties of both continuous and discontinuous formulations. Ainsworth [3] and Ainsworth et al. [4] analyzed these properties for high-order DG finite element methods, considering linear advection and secondorder wave equations respectively. One of the results of these studies is that it may be advantageous to increase the order of the method instead of refining the mesh. An important issue is then to study how to choose the order of polynomial basis function for each element in the computational mesh to obtain a predetermined error. Lähivaara and Huttunen [43] studied such a criterion, connecting the polynomial order $p$ to the mesh parameter 
$\mathrm{h}$, for acoustic and elastic wave equation. Chevaugeon et al. [16] proposed a number of rules to choose an optimal order for the spatial approximation and an optimal time-stepping scheme in order to obtain accurate solutions of the linearized Euler equations in complex geometries and then reduce the size of the problem and the CPU time.

An analysis of the spatial discretization error of a DG method associated with the solutions of $1 \mathrm{D}$ hyperbolic conservation laws has been proposed by Adjerid et al. [1]. In particular, superconvergence results of the DG method at Radau points is obtained. These results are completed by Cheng and Shu [15] who studied the evolution of the space error with time and Zhong and Shu [64] who considered the fully discretized approximation.

In spite of its success in many domains of applications, the DG method has been applied relatively late to seismic wave propagation problems in comparison to other domains of application. If we restrict ourselves to methods designed for the velocity-stress first-order formulation, Käeser and Dumbser [38] presented the ADERDG method for 2D applications that has been widely developped and extended to 3D configurations [26], viscoelastic media [39], applied to a quantitative accuracy analysis [40] or dynamic rupture simulations [52], to mention only a few of the many developments related to this method. The ADER-DG method is based on upwind fluxes and the Cauchy-Kowalewski procedure that automatically provides schemes with the same space and time level accuracy which allow achieving very high levels of convergence. Other references are related to a non-diffusive DG scheme based on centered fluxes and a leap-frog time scheme: Delcourte et al. [25] for the introduction and some properties of the method, Etienne et al. [28] for 3D simulations in a realistic basin, Tago et al. [60] for 3D rupture simulations or Peyrusse et al. [53] for 2D viscoelastic media.

In this paper, we study the P-SV seismic wave propagation considering an isotropic, linearly elastic medium by solving the velocity-stress formulation of the elastodynamic equations. For the discretization of this system, we apply the method described in Delcourte et al. [25], based on centered fluxes and a leap-frog time-discretization. The extension to high-order in space is realized by Lagrange polynomial functions (of degree 0 to 4). Starting with the conclusions of the previous paper [25], we wish to improve some particular points. A numerical convergence study proved that the accuracy of the time scheme is crucial when global high-order accuracy is sought. Indeed, unlike the ADER method that has the same level of accuracy in space and time, the standard leap-frog time scheme, second-order accurate, reduces the global convergence order limiting the interest for high-order space discretizations. Then, we propose an extension of the leap-frog scheme to higher (even) orders of accuracy, following a method proposed for the Maxwell equations by Young [62] and applied in the DG context by Fahs [29]. This method allows us to achieve temporal accuracy to any even order desired, when free surface conditions are applied, by introducing an iterative procedure. Another technique, based on the modified equation approach, has been applied by Agut et al. [2] to construct high-order time schemes for the second-order wave equation. If our analysis is done for a general class of leap-frog schemes, we restrict the numerical application to standard and fourth-order cases, since fourth degree polynomial functions have been considered at the most for spatial discretization. This complete a first introduction of the method, in a two-dimensional context, done by Glinsky et al. [35], especially by adding an analysis of the scheme.

A second limitation of the method found in [25] concerns the low-order absorbing boundary conditions. Indeed, when considering the error evolution during the propagation of a wave in an artificially bounded domain, we can notice a reduction in the convergence due to the absorbing boundary condition. The convergence studies are generally done for problems involving free surface conditions or periodicity conditions, as it is the case for the numerous convergence studies using the ADER method. Our objective here is not to study this point in detail; as mentionned in the review done by Givoli [34], this complex topic is a study in itself. But some simple improvements can be obtained by the evaluation of the absorbing boundary terms at some particular time levels and we wish to study this particular point.

This article is organized as follows. In Section 2, we state the velocity-stress formulation in a symmetric pseudo-conservative form. Then, in Section 3, we detail the discretization of the equations system by the Discontinuous Galerkin method. The high-order leap-frog time scheme as well as the approximation of the boundary conditions are also presented. After, we study, in Section 4, the preservation of a discrete elastodynamic energy and the stability of the scheme, taking into account the free surface or the absorbing boundary conditions. 
Section 5 is devoted to the convergence analysis of the semi-discrete and fully discretized schemes. Finally, we illustrate this study in Section 6 by some basic numerical applications in three dimensions of space, with the aim of validating the 3D solver and studying, numerically, particular points of the method: the propagation of an eigenmode for a convergence study and a comparison between standard and fourth-order leap-frog timeschemes, the propagation of a pulse for a comparison of two different absorbing boundary conditions and the propagation of an explosive source in a half-space. The main objective of this paper being the analysis of the method, applications to 3D more complex configurations, especially in heterogeneous media requiring adapted unstructured meshes, will be the object of a further publication.

\section{Velocity-STRESS FORMUlation In PSEUdo-CONSERVATIVE FORM}

The P-SV wave propagation in an isotropic, linearly elastic medium is modelised by the elastodynamic equations, which initially write in displacement-stress formulation; let be $\vec{U}=\left(U_{\alpha}\right)_{\alpha \in\{x, y, z\}}$ the displacement vector and $\overline{\bar{\sigma}}=\left(\sigma_{\alpha, \beta}\right)_{\alpha, \beta \in\{x, y, z\}}$, the stress tensor, then the system reads

$$
\left\{\begin{aligned}
\rho \partial_{t}^{2} \vec{U} & =\nabla \cdot \overline{\bar{\sigma}} \\
\overline{\bar{\sigma}} & =\lambda(\nabla \cdot \vec{U}) \overline{\bar{I}}+\mu\left(\nabla \vec{U}+(\nabla \vec{U})^{t}\right),
\end{aligned}\right.
$$

where $\overline{\bar{I}}$ is the identity matrix, $\rho$ is the density of the medium and $\lambda$ and $\mu$ are the Lamé parameters related to shear and compressional velocities $\left(v_{s}\right.$ and $\left.v_{p}\right)$ in the medium by $v_{s}=\sqrt{\frac{\mu}{\rho}}$ and $v_{p}=\sqrt{\frac{\lambda+2 \mu}{\rho}}$.

We introduce the velocity vector $\vec{V}=\partial_{t} \vec{U}$ in the equation (2.1) and obtain the first-order velocity-stress formulation [61]:

$$
\left\{\begin{aligned}
\rho \partial_{t} \vec{V} & =\nabla \cdot \overline{\bar{\sigma}} \\
\partial_{t} \overline{\bar{\sigma}} & =\lambda(\nabla \cdot \vec{V}) \overline{\bar{I}}+\mu\left(\nabla \vec{V}+(\nabla \vec{V})^{t}\right) .
\end{aligned}\right.
$$

Let $\vec{W}=(\vec{V}, \vec{\sigma})^{t}$ be the vector composed of the velocity components $\vec{V}=\left(V_{x}, V_{y}, V_{z}\right)^{t}$ and the stress components $\vec{\sigma}=\left(\sigma_{x x}, \sigma_{y y}, \sigma_{z z}, \sigma_{x y}, \sigma_{x z}, \sigma_{y z}\right)^{t}$, since the stress tensor is symmetric, then, the system (2.2) can be rewritten as

$$
\partial_{t} \vec{W}+\sum_{\alpha \in\{x, y, z\}} A_{\alpha}(\rho, \lambda, \mu) \partial_{\alpha} \vec{W}=0 .
$$

We choose here not to detail the matrices $A_{\alpha}(\rho, \lambda, \mu)$.

In order to express the system (2.3) in a pseudo conservative form, we introduce the following change of variables on the stress components,

$$
\overrightarrow{\tilde{\sigma}}=\mathbb{T} \vec{\sigma}=\left(\frac{\sigma_{x x}+\sigma_{y y}+\sigma_{z z}}{3}, \frac{2 \sigma_{x x}-\sigma_{y y}-\sigma_{z z}}{3}, \frac{-\sigma_{x x}+2 \sigma_{y y}-\sigma_{z z}}{3}, \sigma_{x y}, \sigma_{x z}, \sigma_{y z}\right) .
$$

So, the system reads

$$
\left\{\begin{aligned}
\rho \partial_{t} \vec{V} & =\sum_{\alpha \in\{x, y, z\}} \mathbb{M}_{\alpha} \partial_{\alpha} \overrightarrow{\tilde{\sigma}} \\
\Lambda_{0}(\lambda, \mu) \partial_{t} \overrightarrow{\tilde{\sigma}} & =\sum_{\alpha \in\{x, y, z\}} \mathbb{N}_{\alpha} \partial_{\alpha} \vec{V},
\end{aligned}\right.
$$

where $\Lambda_{0}(\lambda, \mu)=\operatorname{diag}\left(\frac{3}{3 \lambda+2 \mu}, \frac{3}{2 \mu}, \frac{3}{2 \mu}, \frac{1}{\mu}, \frac{1}{\mu}, \frac{1}{\mu}\right)$ is a diagonal matrix containing the characteristics of the medium and

$$
\mathbb{M}_{x}=\left(\begin{array}{llllll}
1 & 1 & 0 & 0 & 0 & 0 \\
0 & 0 & 0 & 1 & 0 & 0 \\
0 & 0 & 0 & 0 & 1 & 0
\end{array}\right), \mathbb{M}_{y}=\left(\begin{array}{llllll}
0 & 0 & 0 & 1 & 0 & 0 \\
1 & 0 & 1 & 0 & 0 & 0 \\
0 & 0 & 0 & 0 & 0 & 1
\end{array}\right), \mathbb{M}_{z}=\left(\begin{array}{cccccc}
0 & 0 & 0 & 0 & 1 & 0 \\
0 & 0 & 0 & 0 & 0 & 1 \\
1 & -1 & -1 & 0 & 0 & 0
\end{array}\right) \text {, }
$$




$$
\mathbb{N}_{x}=\left(\begin{array}{ccc}
1 & 0 & 0 \\
2 & 0 & 0 \\
-1 & 0 & 0 \\
0 & 1 & 0 \\
0 & 0 & 1 \\
0 & 0 & 0
\end{array}\right), \mathbb{N}_{y}=\left(\begin{array}{ccc}
0 & 1 & 0 \\
0 & -1 & 0 \\
0 & 2 & 0 \\
1 & 0 & 0 \\
0 & 0 & 0 \\
0 & 0 & 1
\end{array}\right), \mathbb{N}_{z}=\left(\begin{array}{ccc}
0 & 0 & 1 \\
0 & 0 & -1 \\
0 & 0 & -1 \\
0 & 0 & 0 \\
1 & 0 & 0 \\
0 & 1 & 0
\end{array}\right)
$$

We can notice that now the matrices $\mathbb{M}_{\alpha}$ and $\mathbb{N}_{\alpha}(\alpha=x, y, z)$ are constant and do not depend anymore on the material properties. So, the system (2.5) is a pseudo-conservative formulation of (2.3). At last, we multiply the second equation of (2.5) by the following positive definite symmetric (PDS) matrix

$$
\mathbb{S}=\left(\begin{array}{llllll}
1 & 0 & 0 & 0 & 0 & 0 \\
0 & \frac{2}{3} & \frac{1}{3} & 0 & 0 & 0 \\
0 & \frac{1}{3} & \frac{2}{3} & 0 & 0 & 0 \\
0 & 0 & 0 & 1 & 0 & 0 \\
0 & 0 & 0 & 0 & 1 & 0 \\
0 & 0 & 0 & 0 & 0 & 1
\end{array}\right)
$$

in order to obtain a symmetric system. Therefore, we finally get the symmetric pseudo-conservative formulation:

$$
\left\{\begin{aligned}
\rho \partial_{t} \vec{V} & =\sum_{\alpha \in\{x, y, z\}} \mathbb{M}_{\alpha} \partial_{\alpha} \overrightarrow{\tilde{\sigma}} \\
\Lambda(\lambda, \mu) \partial_{t} \overrightarrow{\tilde{\sigma}} & =\sum_{\alpha \in\{x, y, z\}} \mathbb{M}_{\alpha}^{t} \partial_{\alpha} \vec{V}
\end{aligned}\right.
$$

where $\Lambda(\lambda, \mu)=\mathbb{S} \Lambda_{0}(\lambda, \mu)$ is a positive definite symmetric matrix whose spectrum is

$$
S p\{\Lambda\}=\left\{\frac{1}{2 \mu}, \frac{3}{2 \mu}, \frac{1}{\mu}, \frac{1}{\mu}, \frac{1}{\mu}, \frac{3}{3 \lambda+2 \mu}\right\} \subset \mathbb{R}_{+}^{*}
$$

and we can notice that $\mathbb{S N}_{\alpha}=\mathbb{M}_{\alpha}^{t}(\alpha=x, y, z)$. This formulation will be very useful to establish the energy preservation. Then, we add:

(i) a physical boundary condition on the free surface

$$
\overline{\bar{\sigma}} \overrightarrow{\mathbf{n}}=\overrightarrow{0},
$$

which will be rewritten by respect to $\overrightarrow{\tilde{\sigma}}$ in Section 3.3 .

(ii) absorbing boundary conditions

$$
\mathbb{P} \overrightarrow{\tilde{\sigma}}=-\mathbb{A} \vec{V} \text { and } \mathbb{P}^{t} \vec{V}=-\mathbb{B} \overrightarrow{\tilde{\sigma}},
$$

(where the matrices $\mathbb{P}, \mathbb{A}$ and $\mathbb{B}$ will be specified in Sect. 3.3) to approximate an infinite domain.

At last, we need to impose initial data

$$
\vec{V}(0)=\vec{V}_{0} \text { and } \overrightarrow{\tilde{\sigma}}(0)=\overrightarrow{\tilde{\sigma}}_{0},
$$

to ensure the existence and the uniqueness of the solution $(\vec{V}, \overrightarrow{\tilde{\sigma}})$ of the system $(2.6)-(2.9)$.

\section{A discontinuous Galerkin method Combined With a Leap-Frog Scheme}

\subsection{Integration on a simplex}

We consider a bounded polyhedral domain $\Omega$ of $\mathbb{R}^{3}$, discretized in $N_{T}$ tetrahedra $\mathcal{T}_{i}$, which form a partition of the domain. We assume that the characteristics of the medium are constant over each element $\mathcal{T}_{i}$ and denoted by 
$\left(\rho_{i}, \lambda_{i}, \mu_{i}\right)$. We multiply, in the sense of the scalar product, the first (resp. the second) equation of the problem (2.6) by a vector field $\vec{\varphi}_{i} \in \mathbb{R}^{3}$ (resp. $\vec{\psi}_{i} \in \mathbb{R}^{6}$ ) and we integrate them on each element $\mathcal{T}_{i}$. Then, we apply the Green formula to the right-hand sides:

$$
\begin{aligned}
& \rho_{i} \int_{\mathcal{T}_{i}} \vec{\varphi}_{i}^{t} \partial_{t} \vec{V} \mathrm{~d} v=\sum_{\alpha \in\{x, y, z\}}\left[-\int_{\mathcal{T}_{i}}\left(\partial_{\alpha} \vec{\varphi}_{i}\right)^{t} \mathbb{M}_{\alpha} \overrightarrow{\tilde{\sigma}} \mathrm{d} v+\int_{\partial \mathcal{T}_{i}} \vec{\varphi}_{i}^{t} \mathbb{M}_{\alpha} \overrightarrow{\tilde{\sigma}} n_{\alpha_{i}} \mathrm{~d} s\right], \\
& \int_{\mathcal{T}_{i}} \vec{\psi}_{i}^{t} \Lambda_{i} \partial_{t} \overrightarrow{\tilde{\sigma}} \mathrm{d} v=\sum_{\alpha \in\{x, y, z\}}\left[-\int_{\mathcal{T}_{i}}\left(\partial_{\alpha} \vec{\psi}_{i}\right)^{t} \mathbb{M}_{\alpha}^{t} \vec{V} \mathrm{~d} v+\int_{\partial \mathcal{T}_{i}} \vec{\psi}_{i}^{t} \mathbb{M}_{\alpha}^{t} \vec{V} n_{\alpha_{i}} \mathrm{~d} s\right],
\end{aligned}
$$

where we set $\Lambda_{i}=\Lambda\left(\lambda_{i}, \mu_{i}\right)$ and $\overrightarrow{\mathbf{n}}_{i}=\left(n_{x_{i}}, n_{y_{i}}, n_{z_{i}}\right)^{t}$ represents the outwards unit normal vector to $\mathcal{T}_{i}$.

\subsection{Evaluation of volume integrals}

The approximations of $\vec{V}$ and $\overrightarrow{\tilde{\sigma}}$ are denoted by the fields $\vec{V}_{h}$ and $\overrightarrow{\tilde{\sigma}}_{h}$ which are defined locally on each element $\mathcal{T}_{i}$ and may be discontinuous through the interfaces, so that we set

$$
\forall i, \vec{V}_{h_{\mathcal{T}_{i}}}=\vec{V}_{i} \text { and } \overrightarrow{\tilde{\sigma}}_{h_{\mid \mathcal{T}_{i}}}=\overrightarrow{\tilde{\sigma}}_{i}
$$

Let us denote $\mathcal{P}_{m}\left(\mathcal{T}_{i}\right)$ the set of polynomials over $\mathcal{T}_{i}$ with a degree $m$ and consider the vector fields $\vec{\varphi}_{i j} \in \mathcal{P}_{m}^{3}\left(\mathcal{T}_{i}\right)$ and $\vec{\psi}_{i j} \in \mathcal{P}_{m}^{6}\left(\mathcal{I}_{i}\right)$ whose each component is a Lagrange nodal interpolant, then we can write $\vec{V}_{i}$ and $\overrightarrow{\tilde{\sigma}}_{i}$ as linear combinations of time-dependent fields:

$$
\vec{V}_{i}(x, y, z, t)=\sum_{j=1}^{\text {dof }} \widehat{\mathbb{V}}_{i j}(t) \vec{\varphi}_{i j}(x, y, z) \text { and } \overrightarrow{\tilde{\sigma}}_{i}(x, y, z, t)=\sum_{j=1}^{\text {dof }} \widehat{\sigma}_{i j}(t) \vec{\psi}_{i j}(x, y, z),
$$

where dof denotes the number of degrees of freedom on the element $\mathcal{T}_{i}$. To approximate the volume integrals on $\mathcal{T}_{i}$, we just have to replace the fields $\vec{V}$ and $\overrightarrow{\tilde{\sigma}}$ in $(3.1)$ by $\vec{V}_{h}$ and $\overrightarrow{\tilde{\sigma}}_{h}$.

\subsection{Approximation on faces}

To calculate the integrals on $\partial \mathcal{T}_{i}$ of (3.1), we split this boundary in internal and boundary faces. We denote by $\mathcal{V}(i)$ the set of indices of the neighboring elements of $\mathcal{T}_{i}$ and we note by $S_{i k}$ each internal face common to both elements $\mathcal{T}_{i}$ and $\mathcal{T}_{k}$, i.e. $S_{i k}=\mathcal{T}_{i} \cap \mathcal{T}_{k}$. Moreover, some elements $\mathcal{T}_{i}$ have one or more faces common to the boundary of the domain. The set of the indices $k$ of such faces $S_{i k}=\mathcal{T}_{i} \cap \partial \Omega$ is denoted by $\mathcal{E}(i)$ for absorbing boundaries and $\mathcal{K}(i)$ for free surface boundaries. Remark that, for most elements, $\mathcal{E}(i)$ and $\mathcal{K}(i)$ are empty sets. Therefore, introducing $\mathbb{P}_{i k}=\sum_{\alpha \in\{x, y, z\}} \mathbb{M}_{\alpha} n_{\alpha_{i k}}$, we have:

$$
\begin{aligned}
\sum_{\alpha \in\{x, y, z\}} \int_{\partial \mathcal{T}_{i}} \vec{\varphi}_{i}^{t} \mathbb{M}_{\alpha} \overrightarrow{\tilde{\sigma}} n_{\alpha_{i}} \mathrm{~d} s & =\sum_{k \in \mathcal{V}(i)} \int_{S_{i k}} \vec{\varphi}_{i}^{t} \mathbb{P}_{i k} \overrightarrow{\tilde{\sigma}} \mathrm{d} s+\sum_{k \in \mathcal{E}(i)} \int_{S_{i k}} \vec{\varphi}_{i}^{t} \mathbb{P}_{i k} \overrightarrow{\tilde{\sigma}} \mathrm{d} s+\sum_{k \in \mathcal{K}(i)} \int_{S_{i k}} \vec{\varphi}_{i}^{t} \mathbb{P}_{i k} \overrightarrow{\tilde{\sigma}} \mathrm{d} s \\
\sum_{\alpha \in\{x, y, z\}} \int_{\partial \mathcal{T}_{i}} \vec{\psi}_{i}^{t} \mathbb{M}_{\alpha}^{t} \vec{V} n_{\alpha_{i}} \mathrm{~d} s & =\sum_{k \in \mathcal{V}(i)} \int_{S_{i k}} \vec{\psi}_{i}^{t} \mathbb{P}_{i k}^{t} \vec{V} \mathrm{~d} s+\sum_{k \in \mathcal{E}(i)} \int_{S_{i k}} \vec{\psi}_{i}^{t} \mathbb{P}_{i k}^{t} \vec{V} \mathrm{~d} s+\sum_{k \in \mathcal{K}(i)} \int_{S_{i k}} \vec{\psi}_{i}^{t} \mathbb{P}_{i k}^{t} \vec{V} \mathrm{~d} s
\end{aligned}
$$

where $\overrightarrow{\mathbf{n}}_{i k}=\left(n_{x_{i k}}, n_{y_{i k}}, n_{z_{i k}}\right)^{t}$ represents the unit normal vector of $S_{i k}$, oriented from $\mathcal{T}_{i}$ towards $\mathcal{T}_{k}$.

For the interior faces $S_{i k}$ of $\mathcal{T}_{i}(k \in \mathcal{V}(i))$, we choose to apply centered fluxes by introducing the mean-value on this face:

$$
\vec{V}_{S_{i k}} \simeq \frac{1}{2}\left(\vec{V}_{i}+\vec{V}_{k}\right) \quad \text { and } \quad \overrightarrow{\tilde{\sigma}}_{S_{i k}} \simeq \frac{1}{2}\left(\overrightarrow{\tilde{\sigma}}_{i}+\overrightarrow{\tilde{\sigma}}_{k}\right)
$$


For the boundary integrals, two types of boundary conditions have been considered: a free surface condition at the physical interface between air and the medium, and an absorbing condition on the artificial boundaries of an infinite domain.

Free surfaces: On these faces, we compute the fluxes by introducing weakly the physical condition (2.7) in the third term of (3.4a) and the physical condition is rewritten in the new variable $\overrightarrow{\tilde{\sigma}}$ via the change of variables (2.4). No physical condition is applied to the velocity in the third term of (3.4b). So, for a boundary face $S_{i k}$ of $\mathcal{T}_{i}$, with $k \in \mathcal{K}(i)$, this condition reduces to:

$$
\vec{V}_{S_{i k}} \simeq \vec{V}_{i} \text { and } \quad \mathbb{P}_{i k} \overrightarrow{\tilde{\sigma}}_{\left.\right|_{S_{i k}}}=\overrightarrow{0} .
$$

Absorbing surfaces: To simulate infinite domains, we introduce artificial boundaries, on which we impose absorbing conditions. Therefore, for any $k \in \mathcal{E}(i)$ and any real unit vector $\overrightarrow{\mathbf{n}}_{i k}$, we define the matrix

$$
\mathcal{A}_{n_{i k}}\left(\rho_{i}, \lambda_{i}, \mu_{i}\right)=\sum_{\alpha \in\{x, y, z\}} A_{\alpha}\left(\rho_{i}, \lambda_{i}, \mu_{i}\right) n_{\alpha_{i k}}=-\left(\begin{array}{cc}
0_{\mathbb{R}^{3 \times 3}} & \frac{1}{\rho_{i}} \mathbb{P}_{i k} \\
\Lambda_{i}^{-1} \mathbb{P}_{i k}^{t} & 0_{\mathbb{R}^{6 \times 6}}
\end{array}\right),
$$

which is diagonalizable in $\mathbb{R}$, i.e. all its eigenvalues $e_{k}(k=1, \ldots, 9)$ are real:

$$
\begin{gathered}
e_{1}=-v_{p_{i}}, \quad e_{2}=e_{3}=-v_{s_{i}}, \quad e_{4}=e_{5}=e_{6}=0, \quad e_{7}=e_{8}=v_{s_{i}}, \quad e_{9}=v_{p_{i}}, \\
\text { with } v_{p_{i}}=v_{p}\left(\rho_{i}, \lambda_{i}, \mu_{i}\right)=\sqrt{\frac{\lambda_{i}+2 \mu_{i}}{\rho_{i}}} \text { and } v_{s_{i}}=v_{s}\left(\rho_{i}, \lambda_{i}, \mu_{i}\right)=\sqrt{\frac{\mu_{i}}{\rho_{i}}}
\end{gathered}
$$

and we note by $\mathcal{P}_{n}(\rho, \lambda, \mu)$ the matrix whose column $k$ is the right eigenvector associated to the eigenvalue $e_{k}$ :

$$
\left(\begin{array}{ccccccccc}
3 v_{p} n_{x} & -v_{s} n_{x} n_{y} & v_{s} n_{0,1,-1} & 0 & 0 & 0 & -v_{s} n_{0,1,-1} & v_{s} n_{x} n_{y} & -3 v_{p} n_{x} \\
3 v_{p} n_{y} & v_{s} n_{1,0,-1} & -v_{s} n_{x} n_{y} & 0 & 0 & 0 & v_{s} n_{x} n_{y} & -v_{s} n_{1,0,-1} & -3 v_{p} n_{y} \\
3 v_{p} n_{z} & v_{s} n_{y} n_{z} & v_{s} n_{x} n_{z} & 0 & 0 & 0 & -v_{s} n_{x} n_{z} & -v_{s} n_{y} n_{z} & -3 v_{p} n_{z} \\
3 \lambda+2 \mu & 0 & 0 & -n_{x} n_{0,1,1} & -n_{y} n_{1,0,1} & -n_{z} n_{1,1,0} & 0 & 0 & 3 \lambda+2 \mu \\
2 \mu n_{2,-1,-1} & -2 \mu n_{x}^{2} n_{y} & 2 \mu n_{x} n_{0,1,-1} & n_{x} n_{0,1,1} & n_{y} n_{1,0,-2} & n_{z} n_{1,-2,0} & 2 \mu n_{x} n_{0,1,-1} & -2 \mu n_{x}^{2} n_{y} & 2 \mu n_{2,-1,-1} \\
-2 \mu n_{1,-2,1} & 2 \mu n_{y} n_{1,0,-1} & -2 \mu n_{x} n_{y}^{2} & n_{x} n_{0,1,-2} & n_{y} n_{1,0,1} & n_{z} n_{-2,1,0} & -2 \mu n_{x} n_{y}^{2} & 2 \mu n_{y} n_{1,0,-1} & -2 \mu n_{1,-2,1} \\
6 \mu n_{x} n_{y} & \mu n_{x} n_{1,-1,-1} & \mu n_{y} n_{-1,1,-1} & 0 & 0 & 3 n_{x} n_{y} n_{z} & \mu n_{y} n_{-1,1,-1} \mu n_{x} n_{1,-1,-1} & 6 \mu n_{x} n_{y} \\
6 \mu n_{x} n_{z} & 0 & \mu n_{z} n_{1,1,-1} & 0 & 3 n_{x} n_{y} n_{z} & 0 & \mu n_{z} n_{1,1,-1} & 0 & 6 \mu n_{x} n_{z} \\
6 \mu n_{y} n_{z} & \mu n_{z} n_{1,1,-1} & 0 & 3 n_{x} n_{y} n_{z} & 0 & 0 & 0 & \mu n_{z} n_{1,1,-1} & 6 \mu n_{y} n_{z}
\end{array}\right)
$$

where, to simplify, we set $n_{a, b, c}=a n_{x}^{2}+b n_{y}^{2}+c n_{z}^{2}$. Then, the boundary absorbing conditions consist in an upwind technique where we only take into account the outgoing waves neglecting the ingoing waves part. For that, we approximate $\mathcal{A}_{n_{i k}}$ by the matrix $\mathcal{A}_{n_{i k}}^{+}$where we set $\mathcal{A}_{n_{i k}}^{+}=\left(\mathcal{P}_{n_{i k}} \mathcal{D}^{+} \mathcal{P}_{n_{i k}}^{-1}\right)\left(\rho_{i}, \lambda_{i}, \mu_{i}\right)$ with $\mathcal{D}^{+}\left(\rho_{i}, \lambda_{i}, \mu_{i}\right)$ the diagonal matrix composed by the positive eigenvalues of $\mathcal{A}_{n_{i k}}$. Therefore, according to (3.7), we have to approximate

$$
\left(\begin{array}{c}
\mathbb{P}_{i k} \overrightarrow{\tilde{\sigma}} \\
\mathbb{P}_{i k}^{t} \vec{V}
\end{array}\right)=-\left(\begin{array}{cc}
\rho_{i} \overline{\bar{I}}_{3} & 0_{\mathbb{R}^{3 \times 6}} \\
0_{\mathbb{R}^{6 \times 3}} & \Lambda_{i}
\end{array}\right) \mathcal{A}_{n_{i k}}\left(\rho_{i}, \lambda_{i}, \mu_{i}\right)\left(\begin{array}{l}
\vec{V} \\
\overrightarrow{\tilde{\sigma}}
\end{array}\right)
$$

where $\overline{\bar{I}}_{3}$ denotes the identity matrix, by

$$
\left(\begin{array}{c}
\mathbb{P}_{i k} \overrightarrow{\tilde{\sigma}} \\
\mathbb{P}_{i k}^{t} \vec{V}
\end{array}\right) \simeq-\left(\begin{array}{cc}
\rho_{i} \overline{\bar{I}}_{3} & 0_{\mathbb{R}^{3 \times 6}} \\
0_{\mathbb{R}^{6 \times 3}} & \Lambda_{i}
\end{array}\right) \mathcal{A}_{n_{i k}}^{+}\left(\rho_{i}, \lambda_{i}, \mu_{i}\right)\left(\begin{array}{c}
\vec{V}_{i} \\
\overrightarrow{\tilde{\sigma}}_{i}
\end{array}\right) .
$$


After calculus, we obtain:

$$
\left(\begin{array}{cc}
\rho_{i} \overline{\bar{I}}_{3} & 0_{\mathbb{R}^{3 \times 6}} \\
0_{\mathbb{R}^{6 \times 3}} & \Lambda_{i}
\end{array}\right) \mathcal{A}_{n_{i k}}^{+}\left(\rho_{i}, \lambda_{i}, \mu_{i}\right)=\frac{1}{2}\left(\begin{array}{cc}
\mathbb{A}_{i k} & -\mathbb{P}_{i k} \\
-\mathbb{P}_{i k}^{t} & \mathbb{B}_{i k}
\end{array}\right)
$$

where

$$
\mathbb{A}_{i k}=\rho_{i}\left(\begin{array}{ccc}
\left(v_{p_{i}}-v_{s_{i}}\right) n_{x_{i k}}^{2}+v_{s_{i}} & \left(v_{p_{i}}-v_{s_{i}}\right) n_{x_{i k}} n_{y_{i k}} & \left(v_{p_{i}}-v_{s_{i}}\right) n_{x_{i k}} n_{z_{i k}} \\
\left(v_{p_{i}}-v_{s_{i}}\right) n_{x_{i k}} n_{y_{i k}} & \left(v_{p_{i}}-v_{s_{i}}\right) n_{y_{i k}}^{2}+v_{s_{i}} & \left(v_{p_{i}}-v_{s_{i}}\right) n_{y_{i k}} n_{z_{i k}} \\
\left(v_{p_{i}}-v_{s_{i}}\right) n_{x_{i k}} n_{z_{i k}} & \left(v_{p_{i}}-v_{s_{i}}\right) n_{y_{i k}} n_{z_{i k}} & \left(v_{p_{i}}-v_{s_{i}}\right) n_{z_{i k}}^{2}+v_{s_{i}}
\end{array}\right)
$$

and

$$
\begin{aligned}
& \mathbb{B}_{i k}= \frac{1}{\rho_{i} v_{s_{i}}}\left(\begin{array}{cccccc}
1 & n_{x_{i k}}^{2}-n_{z_{i k}}^{2} & n_{y_{i k}}^{2}-n_{z_{i k}}^{2} & 2 n_{x_{i k}} n_{y_{i k}} & 2 n_{x_{i k}} n_{z_{i k}} & 2 n_{y_{i k}} n_{z_{i k}} \\
n_{x_{i k}}^{2}-n_{z_{i k}}^{2} & n_{x_{i k}}^{2}+n_{z_{i k}}^{2} & n_{z_{i k}}^{2} & n_{x_{i k}} n_{y_{i k}} & 0 & -n_{y_{i k}} n_{z_{i k}} \\
n_{y_{i k}}^{2}-n_{z_{i k}}^{2} & n_{z_{i k}}^{2} & n_{y_{i k}}^{2}+n_{z_{i k}}^{2} & n_{x_{i k}} n_{y_{i k}} & -n_{x_{i k}} n_{z_{i k}} & 0 \\
2 n_{x_{i k}} n_{y_{i k}} & n_{x_{i k}} n_{y_{i k}} & n_{x_{i k}} n_{y_{i k}} & n_{x_{i k}}^{2}+n_{y_{i k}}^{2} & n_{y_{i k}} n_{z_{i k}} & n_{x_{i k}} n_{z_{i k}} \\
2 n_{x_{i k}} n_{z_{i k}} & 0 & -n_{x_{i k}} n_{z_{i k}} & n_{y_{i k}} n_{z_{i k}} & n_{x_{i k}}^{2}+n_{z_{i k}}^{2} & n_{x_{i k}} n_{y_{i k}} \\
2 n_{y_{i k}} n_{z_{i k}} & -n_{y_{i k}} n_{z_{i k}} & 0 & n_{x_{i k}} n_{z_{i k}} & n_{x_{i k}} n_{y_{i k}} & n_{y_{i k}}^{2}+n_{z_{i k}}^{2}
\end{array}\right) \\
&+\frac{v_{s_{i}}-v_{p_{i}}}{\rho_{i} v_{p_{i}} v_{s_{i}}}\left(\begin{array}{c}
n_{x_{i k}}^{2}-n_{z_{i k}}^{2} \\
n_{y_{i k}}^{2}-n_{z_{i k}}^{2} \\
2 n_{x_{i k}} n_{y_{i k}}^{2} \\
2 n_{x_{i k}} n_{z_{i k}} \\
2 n_{y_{i k}} n_{z_{i k}}
\end{array}\right) \otimes\left(\begin{array}{c}
1 \\
n_{x_{i k}}^{2}-n_{z_{i k}}^{2} \\
n_{y_{i k}}^{2}-n_{z_{i k}}^{2} \\
2 n_{x_{i k}} n_{y_{i k}} \\
2 n_{x_{i k}} n_{z_{i k}} \\
2 n_{y_{i k}} n_{z_{i k}}
\end{array}\right) .
\end{aligned}
$$

Finally, for a boundary face $S_{i k}$ of $\mathcal{T}_{i}$ with $k \in \mathcal{E}(i)$, the fluxes are approximated in (3.4) by:

$$
\begin{gathered}
\int_{S_{i k}} \vec{\varphi}_{i}^{t} \mathbb{P}_{i k} \overrightarrow{\tilde{\sigma}} \mathrm{d} s \simeq \int_{S_{i k}} \frac{\vec{\varphi}_{i}^{t}}{2}\left[-\mathbb{A}_{i k} \vec{V}_{i}+\mathbb{P}_{i k} \overrightarrow{\tilde{\sigma}}_{i}\right] \mathrm{d} s \\
\int_{S_{i k}} \vec{\psi}_{i}^{t} \mathbb{P}_{i k}^{t} \vec{V} \mathrm{~d} s \simeq \int_{S_{i k}} \frac{\vec{\psi}_{i}^{t}}{2}\left[\mathbb{P}_{i k}^{t} \vec{V}_{i}-\mathbb{B}_{i k} \overrightarrow{\tilde{\sigma}}_{i}\right] \mathrm{d} s .
\end{gathered}
$$

Remark 3.1. The absorbing boundary conditions (2.8) follow from (3.10)-(3.11). It is a first-order approximation, efficient for waves with a normal incidence to the artificial boundaries but whose accuracy could be improved especially to limit reflections in presence of grazing waves.

Remark 3.2. $\mathbb{A}_{i k}$ is a symmetric positive definite matrix whose spectrum is

$$
S p\left(\mathbb{A}_{i k}\right)=\left\{\rho_{i} v_{p_{i}}, \rho_{i} v_{s_{i}}, \rho_{i} v_{s_{i}}\right\},
$$

whereas $\mathbb{B}_{i k}$ is a symmetric semi-definite positive matrix. Indeed, we have

$$
\operatorname{det}\left(\mathbb{B}_{i k}-x \overline{\bar{I}}_{6}\right)=x^{3}\left(x^{3}+a x^{2}+b x+c\right)
$$

where

$$
\begin{aligned}
a= & -\frac{1}{\rho_{i} v_{p_{i}} v_{s_{i}}}\left[v_{p_{i}}\left(2-2 n_{y_{i k}}^{2}+n_{z_{i k}}^{2}-n_{z_{i k}}^{4}+2 n_{y_{i k}}^{4}+2 n_{y_{i k}}^{2} n_{z_{i k}}^{2}\right)\right. \\
& \left.+v_{s_{i}}\left(2+n_{z_{i k}}^{4}-2 n_{y_{i k}}^{4}+2 n_{y_{i k}}^{2}-2 n_{y_{i k}}^{2} n_{z_{i k}}^{2}\right)\right]
\end{aligned}
$$




$$
\begin{aligned}
b= & \frac{1}{\rho_{i}^{2} v_{p_{i}} v_{s_{i}}^{2}}\left[v_{p_{i}}\left(1+2 n_{y_{i k}}^{4}-2 n_{y_{i k}}^{2}-n_{z_{i k}}^{4}+n_{z_{i k}}^{2}-n_{y_{i k}}^{2} n_{z_{i k}}^{2}+3 n_{y_{i k}}^{2} n_{z_{i k}}^{4}+3 n_{y_{i k}}^{4} n_{z_{i k}}^{2}\right)\right. \\
& \left.+v_{s_{i}}\left(4-n_{y_{i k}}^{2}+n_{y_{i k}}^{4}+2 n_{y_{i k}}^{2}+4 n_{y_{i k}}^{2} n_{z_{i k}}^{2}-3 n_{y_{i k}}^{2} n_{z_{i k}}^{4}-3 n_{y_{i k}}^{4} n_{z_{i k}}^{2}+3 n_{y_{i k}}^{4} n_{z_{i k}}^{2}\right)\right] \\
c= & -\frac{1}{\rho_{i}^{3} v_{p_{i}} v_{s_{i}}^{2}}\left(2+2 n_{z_{i k}}^{2}-3 n_{y_{i k}}^{2}+3 n_{y_{i k}}^{4}-n_{z_{i k}}^{4}-n_{y_{i k}}^{2} n_{z_{i k}}^{2}+4 n_{y_{i k}}^{4} n_{z_{i k}}^{2}+4 n_{y_{i k}}^{2} n_{z_{i k}}^{4}\right) .
\end{aligned}
$$

We can deduce that 0 is a third order eigenvalue of $\mathbb{B}_{i k}$, but the three other eigenvalues are not easily specified. Then, we shall use the Sylvester's criteria. For that, we denote by $\mathbb{B}_{i k}=\left(b_{j l}\right)_{1 \leq j, l \leq 6}$ the coefficients of the matrix $\mathbb{B}_{i k}$ and $\mathbb{B}_{q}=\left(b_{j l}\right)_{1 \leq j, l \leq q}$ the submatrices of $\mathbb{B}_{i k}$, and we observe that

$$
\begin{aligned}
& \operatorname{det}\left(\mathbb{B}_{1}\right)=\frac{1}{\rho_{i} v_{p_{i}}}>0, \quad \operatorname{det}\left(\mathbb{B}_{2}\right)=\frac{1}{\rho_{i}^{2} v_{p_{i}} v_{s_{i}}}\left[4 n_{x_{i k}}^{2} n_{z_{i k}}^{2}+n_{y_{i k}}^{2}\left(n_{x_{i k}}^{2}+n_{z_{i k}}^{2}\right)\right] \geq 0, \\
& \operatorname{det}\left(\mathbb{B}_{3}\right)=\frac{9}{\rho_{i}^{3} v_{p_{i}} v_{s_{i}}^{2}} n_{x_{i k}}^{2} n_{y_{i k}}^{2} n_{z_{i k}}^{2} \geq 0, \quad \operatorname{det}\left(\mathbb{B}_{4}\right)=\operatorname{det}\left(\mathbb{B}_{5}\right)=\operatorname{det}\left(\mathbb{B}_{6}\right)=0,
\end{aligned}
$$

so that the three other eigenvalues of $\mathbb{B}_{i k}$ are necessarily positive.

\subsection{Time-discretization}

This subsection is devoted to the high-order leap-frog time-integration of the scheme in the same spirit as [29] for the Maxwell equations.

\subsubsection{The semi-discretized scheme}

Taking into account of (3.2), (3.5), (3.6), (3.14) and (3.15) in the equations (3.1a) and (3.1b), we obtain the following semi-discretized scheme for all $\mathcal{T}_{i}$, with $i=1, \ldots, N_{T}$ :

$$
\begin{aligned}
\rho_{i} \int_{\mathcal{T}_{i}} \vec{\varphi}_{i}^{t} \partial_{t} \vec{V}_{i} \mathrm{~d} v= & -\sum_{\alpha \in\{x, y, z\}} \int_{\mathcal{T}_{i}}\left(\partial_{\alpha} \vec{\varphi}_{i}\right)^{t} \mathbb{M}_{\alpha} \overrightarrow{\tilde{\sigma}}_{i} \mathrm{~d} v+\sum_{k \in \mathcal{V}(i)} \int_{S_{i k}} \vec{\varphi}_{i}^{t} \mathbb{P}_{i k} \frac{\overrightarrow{\tilde{\sigma}}_{i}+\overrightarrow{\tilde{\sigma}}_{k}}{2} \mathrm{~d} s \\
& +\sum_{k \in \mathcal{E}(i)} \int_{S_{i k}} \vec{\varphi}_{i}^{t}\left[\frac{1}{2} \mathbb{P}_{i k} \overrightarrow{\tilde{\sigma}}_{i}-\frac{1}{2} \mathbb{A}_{i k} \vec{V}_{i}\right] \mathrm{d} s \\
\int_{\mathcal{T}_{i}} \vec{\psi}_{i}^{t} \Lambda_{i} \partial_{t} \overrightarrow{\tilde{\sigma}}_{i} \mathrm{~d} v= & -\sum_{\alpha \in\{x, y, z\}} \int_{\mathcal{T}_{i}}\left(\partial_{\alpha} \vec{\psi}_{i}\right)^{t} \mathbb{M}_{\alpha}^{t} \vec{V}_{i} \mathrm{~d} v+\sum_{k \in \mathcal{V}(i)} \int_{S_{i k}} \vec{\psi}_{i}^{t} \mathbb{P}_{i k}^{t} \frac{\vec{V}_{i}+\vec{V}_{k}}{2} \mathrm{~d} s \\
& +\sum_{k \in \mathcal{E}(i)} \int_{S_{i k}} \vec{\psi}_{i}^{t}\left[\frac{1}{2} \mathbb{P}_{i k}^{t} \vec{V}_{i}-\frac{1}{2} \mathbb{B}_{i k} \overrightarrow{\tilde{\sigma}}_{i}\right] \mathrm{d} s+\sum_{k \in \mathcal{K}(i)} \int_{S_{i k}} \vec{\psi}_{i}^{t} \mathbb{P}_{i k}^{t} \vec{V}_{i} \mathrm{~d} s
\end{aligned}
$$

According to (3.3), for each tetrahedron $\mathcal{T}_{i}$, we denote by $\widehat{\mathbb{V}}_{i}$ and $\widehat{\sigma}_{i}$ the column vectors $\left(\widehat{\mathbb{V}}_{i j}\right)_{1 \leq j \leq \text { dof }}$ and $\left(\widehat{\sigma}_{i j}\right)_{1 \leq j \leq \text { dof }}$ respectively. Replacing $\vec{\varphi}_{i}$ by $\vec{\varphi}_{i j}$ in (3.16) and $\vec{\psi}_{i}$ by $\vec{\psi}_{i j}$ in (3.17) for $1 \leq j \leq$ dof, we obtain the following matricial form:

$$
\begin{aligned}
& M_{\rho_{i}} \partial_{t} \widehat{\mathbb{V}}_{i}=-\sum_{\alpha \in\{x, y, z\}} J_{i}^{\alpha} \widehat{\sigma}_{i}+\sum_{k \in \mathcal{V}(i)}\left(K_{i k} \widehat{\sigma}_{i}+G_{i k} \widehat{\sigma}_{k}\right)+\sum_{k \in \mathcal{E}(i)}\left(K_{i k} \widehat{\sigma}_{i}-\mathscr{A}_{i k} \widehat{\mathbb{V}}_{i}\right), \\
& M_{\Lambda_{i}} \partial_{t} \widehat{\sigma}_{i}=-\sum_{\alpha \in\{x, y, z\}} L_{i}^{\alpha} \widehat{\mathbb{V}}_{i}+\sum_{k \in \mathcal{V}(i)}\left(K_{i k}^{t} \widehat{\mathbb{V}}_{i}-G_{k i}^{t} \widehat{\mathbb{V}}_{k}\right)+\sum_{k \in \mathcal{E}(i)}\left(K_{i k}^{t} \widehat{\mathbb{V}}_{i}-\mathscr{B}_{i k} \widehat{\sigma}_{i}\right)+2 \sum_{k \in \mathcal{K}(i)} K_{i k}^{t} \widehat{\mathbb{V}}_{i},
\end{aligned}
$$


where for all $1 \leq j, l \leq$ dof, we have set

$$
\begin{array}{ll}
\left(M_{\rho_{i}}\right)_{j l}=\rho_{i} \int_{\mathcal{T}_{i}} \vec{\varphi}_{i j}^{t} \vec{\varphi}_{i l} \mathrm{~d} s, & \left(M_{\Lambda i}\right)_{j l}=\int_{\mathcal{T}_{i}} \vec{\psi}_{i j}^{t} \Lambda_{i} \vec{\psi}_{i l} \mathrm{~d} s \\
\left(J_{i}^{\alpha}\right)_{j l}=\int_{\mathcal{T}_{i}}\left(\partial_{\alpha} \vec{\varphi}_{i j}\right)^{t} \mathbb{M}_{\alpha} \vec{\psi}_{i l} \mathrm{~d} s, & \left(L_{i}^{\alpha}\right)_{j l}=\int_{\mathcal{T}_{i}}\left(\partial_{\alpha} \vec{\psi}_{i j}\right)^{t} \mathbb{M}_{\alpha}^{t} \vec{\varphi}_{i l} \mathrm{~d} s \\
\left(K_{i k}\right)_{j l}=\frac{1}{2} \int_{S_{i k}} \vec{\varphi}_{i j}^{t} \mathbb{P}_{i k} \vec{\psi}_{i l} \mathrm{~d} s, & \left(G_{i k}\right)_{j l}=\frac{1}{2} \int_{S_{i k}} \vec{\varphi}_{i j}^{t} \mathbb{P}_{i k} \vec{\psi}_{k l} \mathrm{~d} s \\
\left(\mathscr{A}_{i k}\right)_{j l}=\frac{1}{2} \int_{S_{i k}}^{\vec{\varphi}_{i j}^{t}} \mathbb{A}_{i k} \vec{\varphi}_{i l} \mathrm{~d} s, & \left(\mathscr{B}_{i k}\right)_{j l}=\frac{1}{2} \int_{S_{i k}}^{\vec{\psi}_{i j}^{t}} \mathbb{B}_{i k} \vec{\psi}_{i l} \mathrm{~d} s
\end{array}
$$

and used the equalities $\vec{\psi}_{j l}^{t} \mathbb{P}_{i k}^{t} \vec{\varphi}_{i k}=\vec{\varphi}_{i k}^{t} \mathbb{P}_{i k} \vec{\psi}_{j l}=-\vec{\varphi}_{i k}^{t} \mathbb{P}_{k i} \vec{\psi}_{j l}$, thanks to the orientation from $\mathcal{T}_{i}$ to $\mathcal{T}_{k}$ of $n_{\alpha i k}, \alpha \in\{x, y, z\}$, contained in $\mathbb{P}_{i k}$. On the other hand, thanks to the Green formula, we have the following matricial relation for all $i \in\left[1, N_{T}\right]$ :

$$
-\sum_{\alpha \in\{x, y, z\}}\left(J_{i}^{\alpha}+\left(L_{i}^{\alpha}\right)^{t}\right)+2 \sum_{k \in \mathcal{V}(i)} K_{i k}+2 \sum_{k \in \mathcal{E}(i)} K_{i k}+2 \sum_{k \in \mathcal{K}(i)} K_{i k}=0 .
$$

Setting the column vectors $\widehat{\mathbb{V}}=\left(\widehat{\mathbb{V}}_{i}\right)_{1 \leq i \leq N_{T}}$ and $\widehat{\sigma}=\left(\widehat{\sigma}_{i}\right)_{1 \leq i \leq N_{T}}$ whose sizes are $N_{T} *$ dof, the system (3.18) and (3.19) can be rewritten as:

$$
\left\{\begin{array}{l}
M_{\rho} \partial_{t} \widehat{\mathbb{V}}=Q \widehat{\sigma}-R \widehat{\mathbb{V}} \\
M_{\Lambda} \partial_{t} \widehat{\sigma}=X \widehat{\mathbb{V}}-Y \widehat{\sigma}
\end{array}\right.
$$

where $M_{\rho}, M_{\Lambda}, R$ and $Y$ are $\left(N_{T} *\right.$ dof $) *\left(N_{T} *\right.$ dof $)$ block diagonal matrices such that for $1 \leq i \leq N_{T}$,

$$
\left(M_{\rho}\right)_{i i}=M_{\rho_{i}}, \quad\left(M_{\Lambda}\right)_{i i}=M_{\Lambda i}, \quad R_{i i}=\sum_{k \in \mathcal{E}(i)} \mathscr{A}_{i k}, \quad Y_{i i}=\sum_{k \in \mathcal{E}(i)} \mathscr{B}_{i k} .
$$

More precisely, $M_{\rho}, M_{\Lambda}$ and $R$ are symmetric definite positive matrices, and $Y$ is a symmetric semi-definite positive matrix. On the other hand, $Q$ and $X$ are $\left(N_{T} *\right.$ dof $) *\left(N_{T} *\right.$ dof $)$ block sparse matrices such that for $1 \leq i, j \leq N_{T}$

$$
Q_{i j}=\left\{\begin{array}{l}
-\sum_{\alpha \in\{x, y, z\}} J_{i}^{\alpha}+\sum_{k \in \mathcal{V}(i)} K_{i k}+\sum_{k \in \mathcal{E}(i)} K_{i k}, \quad \text { if } i=j, \\
G_{i j}, \quad \text { if } j \in \mathcal{V}(i) \\
0, \quad \text { elsewhere }
\end{array}\right.
$$

and

$$
X_{i j}=\left\{\begin{array}{l}
-\sum_{\alpha \in\{x, y, z\}} L_{i}^{\alpha}+\sum_{k \in \mathcal{V}(i)} K_{i k}^{t}+\sum_{k \in \mathcal{E}(i)} K_{i k}^{t}+2 \sum_{k \in \mathcal{K}(i)} K_{i k}^{t}, \quad \text { if } i=j, \\
-G_{j i}^{t}, \quad \text { if } j \in \mathcal{V}(i), \\
0, \quad \text { elsewhere. }
\end{array}\right.
$$

Applying (3.21), the following essential property holds:

$$
Q+X^{t}=0
$$

\subsubsection{High-order leap-frog scheme}

Then, we apply a high-order leap-frog time-integration scheme. It is an explicit scheme which results, when combined to the centered fluxes defined at (3.5), in a non-diffusive scheme (see Sect. 4.2). We note by $\Delta t$ the time-step and the superscripts refer to time stations. Thus, $\widehat{\mathbb{V}}^{n}$ represents the velocity field at $t_{n}=n \Delta t$ and 
$\widehat{\sigma}^{n+\frac{1}{2}}$ the stress tensor components at $t_{n+\frac{1}{2}}=\left(n+\frac{1}{2}\right) \Delta t$. Note that linear combinations (3.3) are still valid with superscripts. According to Young [62], the leap-frog integration scheme with an order $N$ (even) is written:

$$
\left(\begin{array}{c}
\widehat{\mathbb{V}}\left(t_{n+1}\right) \\
\widehat{\sigma}\left(t_{n+\frac{3}{2}}\right)
\end{array}\right)=\left(\begin{array}{c}
\widehat{\mathbb{V}}\left(t_{n}\right) \\
\widehat{\sigma}\left(t_{n+\frac{1}{2}}\right)
\end{array}\right)+2 \sum_{m=1 \text { (odd) }}^{N-1} \frac{1}{m !}\left(\frac{\Delta t}{2}\right)^{m} \partial_{t}^{m}\left(\begin{array}{c}
\widehat{\mathbb{V}}\left(t_{n+\frac{1}{2}}\right) \\
\widehat{\sigma}\left(t_{n+1}\right)
\end{array}\right) .
$$

As $\widehat{\mathbb{V}}$ and $\widehat{\sigma}$ are not defined at $t_{n+\frac{1}{2}}$ and $t_{n+1}$ respectively, we introduce the linear combinations $\widehat{\mathbb{V}}^{\left[n+\frac{1}{2}\right]}$ and $\widehat{\sigma}^{[n+1]}$ : consider $\alpha \in \mathbb{N}^{\star}$, then we denote by

$$
\widehat{\mathbb{V}}^{\left[n+\frac{1}{2}\right]}=\sum_{i=1}^{\alpha} b_{i} \widehat{\mathbb{V}}^{n+1-i} \quad \text { and } \quad \widehat{\sigma}^{[n+1]}=\sum_{i=1}^{\alpha} b_{i} \widehat{\sigma}^{n+\frac{3}{2}-i},
$$

where the coefficients $b_{i}$ satisfy the following linear Vandermonde system whose solution is unique:

$$
\sum_{i=1}^{\alpha} b_{i}=1 \quad \text { and } \quad \sum_{i=1}^{\alpha} b_{i}\left(\frac{1}{2}-i\right)^{k}=0, \quad \forall k \in\{1, \ldots, \alpha-1\} \text { when } \alpha \geq 2 .
$$

For example, we obtain for $\alpha \in\{1,2,3,4\}$ :

$$
\begin{aligned}
& \widehat{\mathbb{V}}\left[n+\frac{1}{2}\right]=\widehat{\mathbb{V}}^{n}, \quad \widehat{\mathbb{V}}^{\left[n+\frac{1}{2}\right]}=\frac{3 \widehat{\mathbb{V}}^{n}-\widehat{\mathbb{V}}^{n-1}}{2 \widehat{\mathbb{V}}^{n}, \quad \widehat{\mathbb{V}}^{\left.n+\frac{1}{2}\right]}=\frac{15 \widehat{\mathbb{V}}^{n}-10 \widehat{\mathbb{V}}^{n-1}+3 \widehat{\mathbb{V}}^{n-2}}{8},} \\
& \widehat{\mathbb{V}}^{\left[n+\frac{1}{2}\right]}=\frac{35 \widehat{\mathbb{V}}^{n}-35 \widehat{\mathbb{V}}^{n-1}+21 \widehat{\mathbb{V}}^{n-3}}{16} .
\end{aligned}
$$

The interest of such a choice is that (see (5.54) and (5.55) for more details):

$$
\sum_{i=1}^{\alpha} b_{i} \widehat{\mathbb{V}}\left(t_{n+1-i}\right)=\widehat{\mathbb{V}}\left(t_{n+\frac{1}{2}}\right)+\mathcal{O}\left(\Delta t^{\alpha}\right) \quad \text { and } \quad \sum_{i=1}^{\alpha} b_{i} \widehat{\sigma}\left(t_{n+\frac{3}{2}-i}\right)=\widehat{\sigma}\left(t_{n+1}\right)+\mathcal{O}\left(\Delta t^{\alpha}\right)
$$

On the other hand, using (3.22), for all $m$ (odd), we denote by $\alpha_{m}, \beta_{m}, \gamma_{m}$ and $\delta_{m}$ the matrices computed in the following way:

$$
\partial_{t}^{m}\left(\begin{array}{c}
\widehat{\mathbb{V}} \\
\widehat{\sigma}
\end{array}\right)=\left(\begin{array}{cc}
-M_{\rho}^{-1} R & M_{\rho}^{-1} Q \\
M_{\Lambda}^{-1} X & -M_{\Lambda}^{-1} Y
\end{array}\right)^{m}\left(\begin{array}{c}
\widehat{\mathbb{V}} \\
\widehat{\sigma}
\end{array}\right)=\left(\begin{array}{cc}
\alpha_{m} & \beta_{m} \\
\gamma_{m} & \delta_{m}
\end{array}\right)\left(\begin{array}{c}
\widehat{\mathbb{V}} \\
\widehat{\sigma}
\end{array}\right)
$$

Starting from initial values $\vec{V}_{i}^{0}$ at $t=0$ and $\overrightarrow{\tilde{\sigma}}_{i}^{\frac{1}{2}}$ at $t=\frac{\Delta t}{2}$, the final scheme is explicit and expresses:

$$
\left\{\begin{array}{c}
M_{\rho} \frac{\widehat{\mathbb{V}}^{n+1}-\widehat{\mathbb{V}}^{n}}{\Delta t}=Q_{N} \widehat{\sigma}^{n+\frac{1}{2}}-R_{N} \widehat{\mathbb{V}}^{\left[n+\frac{1}{2}\right]}, \\
M_{\Lambda} \frac{\widehat{\sigma}^{n+\frac{3}{2}}-\widehat{\sigma}^{n+\frac{1}{2}}}{\Delta t}=X_{N} \widehat{\mathbb{V}}^{n+1}-Y_{N} \widehat{\sigma}^{[n+1]},
\end{array}\right.
$$

with the matrices (for $N \in \mathbb{N}^{\star}$ even):

$$
\begin{aligned}
& R_{N}=-\frac{2 M_{\rho}}{\Delta t} \sum_{m=1 \text { (odd) }}^{N-1} \frac{1}{m !}\left(\frac{\Delta t}{2}\right)^{m} \alpha_{m}, \quad Q_{N}=\frac{2 M_{\rho}}{\Delta t} \sum_{m=1 \text { (odd) }}^{N-1} \frac{1}{m !}\left(\frac{\Delta t}{2}\right)^{m} \beta_{m}, \\
& X_{N}=\frac{2 M_{\Lambda}}{\Delta t} \sum_{m=1 \text { (odd) }}^{N-1} \frac{1}{m !}\left(\frac{\Delta t}{2}\right)^{m} \gamma_{m}, \quad Y_{N}=-\frac{2 M_{\Lambda}}{\Delta t} \sum_{m=1 \text { (odd) }}^{N-1} \frac{1}{m !}\left(\frac{\Delta t}{2}\right)^{m} \delta_{m} .
\end{aligned}
$$




\subsubsection{Properties}

Lemma 3.3. For all $N \in \mathbb{N}^{\star}$ (even), the matrices $Q_{N}, X_{N}, R_{N}$ and $Y_{N}$ satisfy the following properties:

$$
Q_{N}+X_{N}^{t}=0, \quad R_{N}=R_{N}^{t}, \quad Y_{N}=Y_{N}^{t} .
$$

Proof. We recursively prove that for all $m \in \mathbb{N}^{\star}$ :

$$
\alpha_{m}^{t} M_{\rho}=M_{\rho} \alpha_{m}, \quad \delta_{m}^{t} M_{\Lambda}=M_{\Lambda} \delta_{m}, \quad M_{\rho} \beta_{m}+\gamma_{m}^{t} M_{\Lambda}=0 .
$$

For $m=1$, as $M_{\rho}, M_{\Lambda}, R, Y$ are symmetric, then (3.32) and (3.26) give:

$$
\alpha_{1}^{t} M_{\rho}=-R=M_{\rho} \alpha_{1}, \quad \delta_{1}^{t} M_{\Lambda}=-Y=M_{\Lambda} \delta_{1}, \quad M_{\rho} \beta_{1}+\gamma_{1}^{t} M_{\Lambda}=Q+X^{t}=0 .
$$

After, we remark that

$$
\left(\begin{array}{ll}
\alpha_{m+1} & \beta_{m+1} \\
\gamma_{m+1} & \delta_{m+1}
\end{array}\right)=\left(\begin{array}{ll}
\alpha_{1} & \beta_{1} \\
\gamma_{1} & \delta_{1}
\end{array}\right)\left(\begin{array}{ll}
\alpha_{m} & \beta_{m} \\
\gamma_{m} & \delta_{m}
\end{array}\right)=\left(\begin{array}{ll}
\alpha_{m} & \beta_{m} \\
\gamma_{m} & \delta_{m}
\end{array}\right)\left(\begin{array}{ll}
\alpha_{1} & \beta_{1} \\
\gamma_{1} & \delta_{1}
\end{array}\right)
$$

Therefore, according to (3.37)-(3.39), we obtain

$$
\begin{aligned}
& \alpha_{m+1}^{t} M_{\rho}=\left(\begin{array}{ll}
\alpha_{1} & \alpha_{m}+\beta_{1} \gamma_{m}
\end{array}\right)^{t} M_{\rho}=\alpha_{m}^{t} \alpha_{1}^{t} M_{\rho}+\gamma_{m}^{t} \beta_{1}^{t} M_{\rho} \\
& =\alpha_{m}^{t} M_{\rho} \alpha_{1}-\gamma_{m}^{t} M_{\Lambda} \gamma_{1}=M_{\rho}\left(\alpha_{m} \alpha_{1}+\beta_{m} \gamma_{1}\right) \\
& =M_{\rho}\left(\alpha_{1} \alpha_{m}+\beta_{1} \gamma_{m}\right)=M_{\rho} \alpha_{m+1}, \\
& \delta_{m+1}^{t} M_{\Lambda}=\left(\gamma_{1} \beta_{m}+\delta_{1} \delta_{m}\right)^{t} M_{\Lambda}=\beta_{m}^{t} \gamma_{1}^{t} M_{\Lambda}+\delta_{m}^{t} \delta_{1}^{t} M_{\Lambda} \\
& =-\beta_{m}^{t} M_{\rho} \beta_{1}+\delta_{m}^{t} M_{\Lambda} \delta_{1}=M_{\Lambda}\left(\gamma_{m} \beta_{1}+\delta_{m} \delta_{1}\right) \\
& =M_{\Lambda}\left(\gamma_{1} \beta_{m}+\delta_{1} \delta_{m}\right) \quad=M_{\Lambda} \delta_{m+1} \text {, } \\
& M_{\rho} \beta_{m+1}+\gamma_{m+1}^{t} M_{\Lambda}=M_{\rho}\left(\alpha_{m} \beta_{1}+\beta_{m} \delta_{1}\right)+\left(\gamma_{1} \alpha_{m}+\delta_{1} \gamma_{m}\right)^{t} M_{\Lambda} \\
& =\left(M_{\rho} \alpha_{m} \beta_{1}+\alpha_{m}^{t} \gamma_{1}^{t} M_{\Lambda}\right)+\left(M_{\rho} \beta_{m} \delta_{1}+\gamma_{m}^{t} \delta_{1}^{t} M_{\Lambda}\right) \\
& =\alpha_{m}^{t}\left(M_{\rho} \beta_{1}+\gamma_{1}^{t} M_{\Lambda}\right)+\left(M_{\rho} \beta_{m}+\gamma_{m}^{t} M_{\Lambda}\right) \delta_{1} \\
& =0 \text {. }
\end{aligned}
$$

Remark 3.4. In the particular case of free surface boundary conditions only (i.e. when $R=0$ and $Y=0$ ), for $m$ odd, we recursively show that:

$$
\alpha_{m}=0, \quad \beta_{m}=M_{\rho}^{-1} Q\left(M_{\Lambda}^{-1} X M_{\rho}^{-1} Q\right)^{\frac{m-1}{2}}, \quad \gamma_{m}=M_{\Lambda}^{-1} X\left(M_{\rho}^{-1} Q M_{\Lambda}^{-1} X\right)^{\frac{m-1}{2}} \text { and } \quad \delta_{m}=0 .
$$

Thus, with a change of variable, we find that $R_{N}=0, Y_{N}=0$ and

$$
\begin{aligned}
Q_{N} & =\sum_{k=0}^{\frac{N}{2}-1} \frac{1}{(2 k+1) !}\left(\frac{\Delta t}{2}\right)^{2 k} Q\left(M_{\Lambda}^{-1} X M_{\rho}^{-1} Q\right)^{k}, \\
X_{N} & =\sum_{k=0}^{\frac{N}{2}-1} \frac{1}{(2 k+1) !}\left(\frac{\Delta t}{2}\right)^{2 k} X\left(M_{\rho}^{-1} Q M_{\Lambda}^{-1} X\right)^{k} .
\end{aligned}
$$

Remark 3.5. As the matrix $R_{2}=R$ is symmetric positive definite and $M_{\rho} \alpha_{3}$ is symmetric, then we always can find a condition under the time step $\Delta t$ such that

$$
R_{4}=R-\frac{\Delta t^{2}}{24} M_{\rho} \alpha_{3}
$$

is a symmetric positive definite matrix. 
Remark 3.6. As the matrix $Y_{2}=Y$ is symmetric positive semi-definite, $M_{\Lambda} \delta_{3}$ is symmetric, and

$$
Y_{4}=Y+\frac{\Delta t^{2}}{24}\left[X M_{\rho}^{-1} R M_{\rho}^{-1} Q+Y M_{\Lambda}^{-1} X M_{\rho}^{-1} Q+X M_{\rho}^{-1} Q M_{\Lambda}^{-1} Y+Y M_{\Lambda}^{-1} Y M_{\Lambda}^{-1} Y\right]
$$

then by considering the eigenvector $v \neq 0$ of $Y$ such that $Y v=0$, we find $v^{t} Y_{4} v<0$. Consequently, $Y_{4}$ is only symmetric (but not positive semi-definite).

\section{ENERGY PRESERVATION AND STABILITy OF THE SCHEME}

The discrete scheme (3.33) being explicit, it is conditionally stable. The aim of this section is to establish a sufficient condition on the time step $\Delta t$ for the $\mathrm{L}^{2}$-stability of the Discontinuous Galerkin scheme, taking into account both free surface (3.6) and absorbing boundary conditions (3.14) and (3.15). Following [10,11,30], we shall define a discrete energy and prove that it is a quadratic positive definite form playing the role of a Lyapunov function of numerical unknowns.

\subsection{A discrete elastodynamic energy}

In the continuum, the total energy of the system is given by:

$$
E(t)=\underbrace{\frac{1}{2} \int_{\Omega} \rho\|\vec{V}(t)\|^{2} \mathrm{~d} v}_{E_{k}, \text { kinetic energy }}+\underbrace{\frac{1}{2} \int_{\Omega} \overline{\bar{\sigma}}(t): \overline{\bar{\epsilon}}(t) \mathrm{d} v}_{E_{m}, \text { mechanical energy }},
$$

where $\overline{\bar{\epsilon}}$ is the symmetric deformation tensor whose components are $\epsilon_{i, j}=\frac{1}{2}\left(\frac{\partial U_{i}}{\partial x_{j}}+\frac{\partial U_{j}}{\partial x_{i}}\right)$. As for the stress, we introduce the deformation vector $\vec{\epsilon}=\left(\epsilon_{x x}, \epsilon_{y y}, \epsilon_{z z}, 2 \epsilon_{x y}, 2 \epsilon_{x z}, 2 \epsilon_{y z}\right)^{t}$. For an elastic medium, the generalized Hooke's law links deformations and stresses through the linear relationship $\vec{\epsilon}=C \vec{\sigma}$, where the matrix $C$ expresses

$$
C=\frac{1}{E}\left(\begin{array}{cccccc}
1 & -\nu & -\nu & 0 & 0 & 0 \\
-\nu & 1 & -\nu & 0 & 0 & 0 \\
-\nu & -\nu & 1 & 0 & 0 & 0 \\
0 & 0 & 0 & 2(1+\nu) & 0 & 0 \\
0 & 0 & 0 & 0 & 2(1+\nu) & 0 \\
0 & 0 & 0 & 0 & 0 & 2(1+\nu)
\end{array}\right)
$$

$E$ and $\nu$ being respectively the Young's modulus and the Poisson's ratio

$$
E=\frac{\mu(3 \lambda+2 \mu)}{\lambda+\mu} \text { and } \quad \nu=\frac{\lambda}{2(\lambda+\mu)} .
$$

Thanks to this relationship, the mechanical energy becomes

$$
E_{m}=\frac{1}{2} \int_{\Omega}(\vec{\sigma})^{t} \vec{\epsilon} \mathrm{d} v=\frac{1}{2} \int_{\Omega}(\vec{\sigma})^{t} C \vec{\sigma} \mathrm{d} v
$$

and introducing the change of variables (2.4), we obtain

$$
E_{m}=\frac{1}{2} \int_{\Omega}\left(\mathbb{T}^{-1} \overrightarrow{\tilde{\sigma}}\right)^{t} C\left(\mathbb{T}^{-1} \overrightarrow{\tilde{\sigma}}\right) \mathrm{d} v=\frac{1}{2} \int_{\Omega}(\overrightarrow{\tilde{\sigma}})^{t}\left[\left(\mathbb{T}^{-1}\right)^{t} C \mathbb{T}^{-1}\right] \overrightarrow{\tilde{\sigma}} \mathrm{d} v .
$$

We can easily check that $\left(\mathbb{T}^{-1}\right)^{t} C \mathbb{T}^{-1}=\Lambda$ so that, the total energy writes

$$
E(t)=\frac{1}{2} \int_{\Omega} \rho\|\vec{V}(t)\|^{2} \mathrm{~d} v+\frac{1}{2} \int_{\Omega}(\overrightarrow{\tilde{\sigma}}(t))^{t} \Lambda(\lambda, \mu) \overrightarrow{\tilde{\sigma}}(t) \mathrm{d} v .
$$


Definition 4.1. The discrete energy $E^{n}$ in three dimensions of space at time $n \Delta t$ is defined by:

$$
E^{n}=\frac{1}{2}\left[\left(\widehat{\mathbb{V}}^{n}\right)^{t} M_{\rho} \widehat{\mathbb{V}}^{n}+\left(\widehat{\sigma}^{n+\frac{1}{2}}\right)^{t} M_{\Lambda} \widehat{\sigma}^{n-\frac{1}{2}}\right] .
$$

\subsection{Energy preservation}

Now, we study the evolution of the discrete elastodynamic energy through one time-step. More precisely, we aim to establish that the combination between the centered approximation of the fluxes and the high-order leapfrog time-integration leads to a non-dissipative scheme for unbounded domains or domains with free surfaces.

Lemma 4.2. For all $N \geq 2$ (even), the variation of the total discrete energy during one time-step $\Delta t$ is

$$
E^{n+1}-E^{n}=-\Delta t\left(\frac{\widehat{\mathbb{V}}^{n+1}+\widehat{\mathbb{V}}^{n}}{2}\right)^{t} R_{N} \widehat{\mathbb{V}}^{\left[n+\frac{1}{2}\right]}-\Delta t\left(\widehat{\sigma}^{n+\frac{1}{2}}\right)^{t} Y_{N} \frac{\widehat{\sigma}^{[n+1]}+\widehat{\sigma}^{[n]}}{2} .
$$

Proof. We calculate the variation of the discrete energy during one time-step $\Delta t$ :

$$
E^{n+1}-E^{n}=\frac{\left(\widehat{\mathbb{V}}^{n+1}+\widehat{\mathbb{V}}^{n}\right)^{t}}{2} M_{\rho}\left(\widehat{\mathbb{V}}^{n+1}-\widehat{\mathbb{V}}^{n}\right)+\frac{\left(\widehat{\sigma}^{n+\frac{1}{2}}\right)^{t}}{2} M_{\Lambda}\left(\widehat{\sigma}^{n+\frac{3}{2}}-\widehat{\sigma}^{n-\frac{1}{2}}\right) .
$$

Using (3.33), we find

$$
E^{n+1}-E^{n}=\Delta t\left(\frac{\widehat{\mathbb{V}}^{n+1}+\widehat{\mathbb{V}}^{n}}{2}\right)^{t}\left[\left(Q_{N}+X_{N}^{t}\right) \widehat{\sigma}^{n+\frac{1}{2}}-R_{N} \widehat{\mathbb{V}}^{\left[n+\frac{1}{2}\right]}\right]-\Delta t\left(\widehat{\sigma}^{n+\frac{1}{2}}\right)^{t} Y_{N} \frac{\widehat{\sigma}^{[n+1]}+\widehat{\sigma}^{[n]}}{2}
$$

We conclude with (3.36).

Corollary 4.3. Using the scheme (3.33), for an infinite domain or a domain including free surface boundaries (3.6) only, the discrete elastodynamic energy is preserved through one time-step:

$$
\forall n \in \mathbb{N}, \quad E^{n+1}=E^{n} .
$$

Proof. In an infinite domain or a domain with free surface boundary conditions, $\mathcal{E}(i)$ is an empty set, so that the matrices $R=0$ and $Y=0$. Therefore, following Remark 3.4 and (4.2), the discrete elastodynamic energy is preserved.

\subsection{A corrected discrete elastodynamic energy for absorbing boundaries}

Let be $\alpha \in \mathbb{N}^{\star}$ and $b_{i}$ as in (3.28), $\forall i \in\{1, \ldots, \alpha\}$. In what follows, we define the signs of $b_{i}$ and $b_{i}+b_{i+1}$ respectively by:

$$
\begin{aligned}
\forall i \in\{1, \ldots, \alpha\}, \quad \gamma_{i} & =\frac{b_{i}}{\left|b_{i}\right|}, \\
\forall i \in\{1, \ldots, \alpha-1\}, \quad s_{i} & =\frac{b_{i}+b_{i+1}}{\left|b_{i}+b_{i+1}\right|} .
\end{aligned}
$$

When absorbing boundary conditions are applied at some faces of the domain (i.e. when $\mathcal{E}(i)$ is not empty), the discrete variation of the energy through one time-step $\Delta t$ is not necessarily negative according to Lemma 4.2 , because of the time-asymmetry on both $\widehat{\mathbb{V}}$ and $\widehat{\sigma}$ in (4.2). In order to overcome this difficulty, we introduce correction terms in the definition of the discrete energy (4.1) and prove that this corrected discrete elastodynamic energy is not increased through each time step. 
Definition 4.4. For $\alpha=1$, we introduce a corrected discrete elastodynamic energy $\mathcal{E}^{n}$ at time $n \Delta t$ by:

$$
\mathcal{E}^{n}=E^{n}-\frac{\Delta t}{4}\left[\left(\widehat{\mathbb{V}}^{n}\right)^{t} R_{N} \widehat{\mathbb{V}}^{n}-\left(\widehat{\sigma}^{n-\frac{1}{2}}\right)^{t} Y_{N} \widehat{\sigma}^{n-\frac{1}{2}}\right] .
$$

Definition 4.5. For $\alpha \in\{2,3,4\}$, we introduce a corrected discrete elastodynamic energy $\mathcal{E}^{n}$ at time $n \Delta t$ by:

$$
\begin{aligned}
\mathcal{E}^{n}= & E^{n}-\frac{\Delta t}{4} \sum_{i=1}^{\alpha}\left|b_{i}\right|\left[\left(\widehat{\mathbb{V}}^{n}\right)^{t} R_{N} \widehat{\mathbb{V}}^{n}-\left(\widehat{\sigma}^{n+\frac{1}{2}-i}\right)^{t} Y_{N} \widehat{\sigma}^{n+\frac{1}{2}-i}\right] \\
& +\frac{\Delta t}{4} \sum_{i=2}^{\alpha}\left|b_{i}\right|\left(\widehat{\mathbb{V}}^{n}-\gamma_{i} \widehat{\mathbb{V}}^{n+1-i}\right)^{t} R_{N}\left(\widehat{\mathbb{V}}^{n}-\gamma_{i} \widehat{\mathbb{V}}^{n+1-i}\right) .
\end{aligned}
$$

These correction terms only concern the absorbing faces and have no particular physical meaning. However, they match the loss of energy (which appears as a decreasing discrete energy) through these faces since we only consider outgoing waves. Using (4.2), we easily prove the following lemma when $\alpha=1$.

Lemma 4.6. For $\alpha=1$, we have for all $n \in \mathbb{N}$ :

$$
\mathcal{E}^{n+1}-\mathcal{E}^{n}=-\frac{\Delta t}{4}\left[\left(\widehat{\mathbb{V}}^{n+1}+\widehat{\mathbb{V}}^{n}\right)^{t} R_{N}\left(\widehat{\mathbb{V}}^{n+1}+\widehat{\mathbb{V}}^{n}\right)+\left(\widehat{\sigma}^{n+\frac{1}{2}}+\widehat{\sigma}^{n-\frac{1}{2}}\right)^{t} Y_{N}\left(\widehat{\sigma}^{n+\frac{1}{2}}+\widehat{\sigma}^{n-\frac{1}{2}}\right)\right] .
$$

Then, we can establish the following result when $\alpha \in\{2,3,4\}$, but it is not valid for $\alpha \geq 5$.

Lemma 4.7. For $\alpha \in\{2,3,4\}$, we have for all $n \in \mathbb{N}$ :

$$
\begin{aligned}
\mathcal{E}^{n+1}-\mathcal{E}^{n}= & -\frac{\Delta t}{4}\left[\sum_{i=1}^{\alpha-1}\left|b_{i}+b_{i+1}\right|\left(\widehat{\mathbb{V}}^{n+1}+s_{i} \widehat{\mathbb{V}}^{n+1-i}\right)^{t} R_{N}\left(\widehat{\mathbb{V}}^{n+1}+s_{i} \widehat{\mathbb{V}}^{n+1-i}\right)\right. \\
& +\left|b_{\alpha}\right|\left(\widehat{\mathbb{V}}^{n+1}+\gamma_{\alpha} \widehat{\mathbb{V}}^{n+1-\alpha}\right)^{t} R_{N}\left(\widehat{\mathbb{V}}^{n+1}+\gamma_{\alpha} \widehat{\mathbb{V}}^{n+1-\alpha}\right) \\
& +\sum_{i=1}^{\alpha-1}\left|b_{i}+b_{i+1}\right|\left(\widehat{\sigma}^{n+\frac{1}{2}}+s_{i} \widehat{\sigma}^{n+\frac{1}{2}-i}\right)^{t} Y_{N}\left(\widehat{\sigma}^{n+\frac{1}{2}}+s_{i} \widehat{\sigma}^{n+\frac{1}{2}-i}\right) \\
& \left.+\left|b_{\alpha}\right|\left(\widehat{\sigma}^{n+\frac{1}{2}}+\gamma_{\alpha} \widehat{\sigma}^{n+\frac{1}{2}-\alpha}\right)^{t} Y_{N}\left(\widehat{\sigma}^{n+\frac{1}{2}}+\gamma_{\alpha} \widehat{\sigma}^{n+\frac{1}{2}-\alpha}\right)\right] .
\end{aligned}
$$

Proof. We denote by $E_{V}^{n}=\frac{1}{2}\left(\widehat{\mathbb{V}}^{n}\right)^{t} M_{\rho} \widehat{\mathbb{V}}^{n}$ and $E_{\sigma}^{n}=\frac{1}{2}\left(\widehat{\sigma}^{n+\frac{1}{2}}\right)^{t} M_{\Lambda} \widehat{\sigma}^{n-\frac{1}{2}}$. Thus, according to (4.1)-(4.2) and the definitions $(3.28)$ of $\widehat{\mathbb{V}}^{\left[n+\frac{1}{2}\right]}$ and $\widehat{\sigma}^{[n+1]}$, we obtain

$$
\begin{aligned}
& E_{V}^{n+1}-E_{V}^{n}=-\frac{\Delta t}{2}\left[\sum_{i=1}^{\alpha} b_{i}\left(\widehat{\mathbb{V}}^{n+1}\right)^{t} R_{N} \widehat{\mathbb{V}}^{n+1-i}+b_{1}\left(\widehat{\mathbb{V}}^{n}\right)^{t} R_{N} \widehat{\mathbb{V}}^{n}+\sum_{i=2}^{\alpha} b_{i}\left(\widehat{\mathbb{V}}^{n}\right)^{t} R_{N} \widehat{\mathbb{V}}^{n+1-i}\right] \\
& E_{\sigma}^{n+1}-E_{\sigma}^{n}=-\frac{\Delta t}{2}\left(\hat{\sigma}^{n+\frac{1}{2}}\right)^{t} Y_{N}\left(b_{1} \widehat{\sigma}^{n+\frac{1}{2}}+\sum_{i=1}^{\alpha-1}\left(b_{i}+b_{i+1}\right) \widehat{\sigma}^{n+\frac{1}{2}-i}+b_{\alpha} \widehat{\sigma}^{n+\frac{1}{2}-\alpha}\right) .
\end{aligned}
$$

Then, we set $\mathcal{E}=\mathcal{E}_{V}+\mathcal{E}_{\sigma}$, with

$$
\begin{aligned}
& \mathcal{E}_{V}^{n}=E_{V}^{n}-\frac{\Delta t}{4}\left[\left|b_{1}\right|\left(\widehat{\mathbb{V}}^{n}\right)^{t} R_{N} \widehat{\mathbb{V}}^{n}-\sum_{i=2}^{\alpha}\left|b_{i}\right|\left(\widehat{\mathbb{V}}^{n+1-i}\right)^{t} R_{N} \widehat{\mathbb{V}}^{n+1-i}+2 \sum_{i=2}^{\alpha} b_{i}\left(\widehat{\mathbb{V}}^{n}\right)^{t} R_{N} \widehat{\mathbb{V}}^{n+1-i}\right], \\
& \mathcal{E}_{\sigma}^{n}=E_{\sigma}^{n}+\frac{\Delta t}{4} \sum_{i=1}^{\alpha}\left|b_{i}\right|\left(\widehat{\sigma}^{n+\frac{1}{2}-i}\right)^{t} Y_{N} \widehat{\sigma}^{n+\frac{1}{2}-i},
\end{aligned}
$$


so that, according to (4.11)-(4.13) and (4.12)-(4.14), we have

$$
\begin{aligned}
\mathcal{E}_{V}^{n+1}-\mathcal{E}_{V}^{n}= & -\frac{\Delta t}{4}\left[\left|b_{1}\right|\left(\widehat{\mathbb{V}}^{n+1}\right)^{t} R_{N} \widehat{\mathbb{V}}^{n+1}+\left(2 b_{1}-\left|b_{1}\right|-\left|b_{2}\right|\right)\left(\widehat{\mathbb{V}}^{n}\right)^{t} R_{N} \widehat{\mathbb{V}}^{n}\right. \\
& +\sum_{i=2}^{\alpha-1}\left(\left|b_{i}\right|-\left|b_{i+1}\right|\right)\left(\widehat{\mathbb{V}}^{n+1-i}\right)^{t} R_{N} \widehat{\mathbb{V}}^{n+1-i}+\left|b_{\alpha}\right|\left(\widehat{\mathbb{V}}^{n+1-\alpha}\right)^{t} R_{N} \widehat{\mathbb{V}}^{n+1-\alpha} \\
& \left.+2 \sum_{i=1}^{\alpha-1}\left(b_{i}+b_{i+1}\right)\left(\widehat{\mathbb{V}}^{n+1}\right)^{t} R_{N} \widehat{\mathbb{V}}^{n+1-i}+2 b_{\alpha}\left(\widehat{\mathbb{V}}^{n+1}\right)^{t} R_{N} \widehat{\mathbb{V}}^{n+1-\alpha}\right], \\
\mathcal{E}_{\sigma}^{n+1}-\mathcal{E}_{\sigma}^{n}= & -\frac{\Delta t}{4}\left[-\left|b_{1}\right|\left(\widehat{\sigma}^{n+\frac{1}{2}}\right)^{t} Y_{N} \widehat{\sigma}^{n+\frac{1}{2}}+\sum_{i=1}^{\alpha-1}\left(\left|b_{i}\right|-\left|b_{i+1}\right|\right)\left(\widehat{\sigma}^{n+\frac{1}{2}-i}\right)^{t} Y_{N} \widehat{\sigma}^{n+\frac{1}{2}-i}\right. \\
& \left.+\left|b_{\alpha}\right|\left(\widehat{\sigma}^{n+\frac{1}{2}-\alpha}\right)^{t} Y_{N} \widehat{\sigma}^{n+\frac{1}{2}-\alpha}\right]+E_{\sigma}^{n+1}-E_{\sigma}^{n} .
\end{aligned}
$$

At last, we use the following identities in (4.12): for $i \in\{1, \ldots, \alpha-1\}$,

$$
\begin{aligned}
2\left(b_{i}+b_{i+1}\right)\left(\widehat{\sigma}^{n+\frac{1}{2}}\right)^{t} Y_{N} \widehat{\sigma}^{n+\frac{1}{2}-i}= & \left|b_{i}+b_{i+1}\right|\left(\widehat{\sigma}^{n+\frac{1}{2}}+s_{i} \widehat{\sigma}^{n+\frac{1}{2}-i}\right)^{t} Y_{N}\left(\widehat{\sigma}^{n+\frac{1}{2}}+s_{i} \widehat{\sigma}^{n+\frac{1}{2}-i}\right) \\
& -\left|b_{i}+b_{i+1}\right|\left[\left(\widehat{\sigma}^{n+\frac{1}{2}}\right)^{t} Y_{N} \widehat{\sigma}^{n+\frac{1}{2}}+\left(\widehat{\sigma}^{n+\frac{1}{2}-i}\right)^{t} Y_{N} \widehat{\sigma}^{n+\frac{1}{2}-i}\right]
\end{aligned}
$$

and similar identities for the two last terms of (4.15). We finally obtain the variation of the total corrected discrete elastodynamic energy:

$$
\begin{aligned}
\mathcal{E}^{n+1}-\mathcal{E}^{n}= & -\frac{\Delta t}{4}\left[2\left(b_{1}-\left|b_{1}\right|\right)\left(\widehat{\mathbb{V}}^{n}\right)^{t} R_{N} \widehat{\mathbb{V}}^{n}+\left(\left|b_{1}\right|-\sum_{i=1}^{\alpha-1}\left|b_{i}+b_{i+1}\right|-\left|b_{\alpha}\right|\right)\left(\widehat{\mathbb{V}}^{n+1}\right)^{t} R_{N} \widehat{\mathbb{V}}^{n+1}\right. \\
& +\left(2 b_{1}-\left|b_{1}\right|-\sum_{i=1}^{\alpha-1}\left|b_{i}+b_{i+1}\right|-\left|b_{\alpha}\right|\right)\left(\widehat{\sigma}^{n+\frac{1}{2}}\right)^{t} Y_{N} \widehat{\sigma}^{n+\frac{1}{2}} \\
& +\sum_{i=1}^{\alpha-1}\left(\left|b_{i}\right|-\left|b_{i}+b_{i+1}\right|-\left|b_{i+1}\right|\right)\left(\left(\widehat{\mathbb{V}}^{n+1-i}\right)^{t} R_{N} \widehat{\mathbb{V}}^{n+1-i}+\left(\widehat{\sigma}^{n+\frac{1}{2}-i}\right)^{t} Y_{N} \widehat{\sigma}^{n+\frac{1}{2}-i}\right) \\
& +\sum_{i=1}^{\alpha-1}\left|b_{i}+b_{i+1}\right|\left(\widehat{\mathbb{V}}^{n+1}+s_{i} \widehat{\mathbb{V}}^{n+1-i}\right)^{t} R_{N}\left(\widehat{\mathbb{V}}^{n+1}+s_{i} \widehat{\mathbb{V}}^{n+1-i}\right) \\
& +\left|b_{\alpha}\right|\left(\widehat{\mathbb{V}}^{n+1}+\gamma_{\alpha} \widehat{\mathbb{V}}^{n+1-\alpha}\right)^{t} R_{N}\left(\widehat{\mathbb{V}}^{n+1}+\gamma_{\alpha} \widehat{\mathbb{V}}^{n+1-\alpha}\right) \\
& +\sum_{i=1}^{\alpha-1}\left|b_{i}+b_{i+1}\right|\left(\widehat{\sigma}^{n+\frac{1}{2}}+s_{i} \widehat{\sigma}^{n+\frac{1}{2}-i}\right)^{t} Y_{N}\left(\widehat{\sigma}^{n+\frac{1}{2}}+s_{i} \widehat{\sigma}^{n+\frac{1}{2}-i}\right) \\
& \left.+\left|b_{\alpha}\right|\left(\widehat{\sigma}^{n+\frac{1}{2}}+\gamma_{\alpha} \widehat{\sigma}^{n+\frac{1}{2}-\alpha}\right)^{t} Y_{N}\left(\widehat{\sigma}^{n+\frac{1}{2}}+\gamma_{\alpha} \widehat{\sigma}^{n+\frac{1}{2}-\alpha}\right)\right] .
\end{aligned}
$$

When $\alpha \in\{2,3,4\}$, we can verify according to (3.30) that $b_{1} \geq 0$, then $b_{1}-\sum_{i=1}^{\alpha-1}\left|b_{i}+b_{i+1}\right|-\left|b_{\alpha}\right|=0$ and $\forall i \in\{1, \ldots, \alpha-1\},\left|b_{i}\right|-\left|b_{i}+b_{i+1}\right|-\left|b_{i+1}\right|=0$. On the contrary, these two last equalities are not satisfied for $\alpha \in\{5,6\}$. 
Corollary 4.8. For $\alpha \in\{1,2,3,4\}$ and $N=2$, the sequel $\left(\mathcal{E}^{n}\right)_{n \in \mathbb{N}}$ is not increasing.

Proof. We use Lemmae 4.6 and 4.7. If $N=2$, then $\mathcal{E}^{n+1}-\mathcal{E}^{n}$ is a negative quantity, because the matrices $R$ and $Y$ are positive definite symmetric and semi-positive definite symmetric respectively. On the contrary, if $N \geq 4$, we are unable to conclude according to Remarks 3.5 and 3.6.

\subsection{Stability of the scheme}

We aim at proving that the discrete corrected elastodynamic energy $\mathcal{E}^{n}$ is a positive definite quadratic form of the unknowns $\widehat{\mathbb{V}}^{n}$ and $\widehat{\sigma}^{n-1 / 2}$ under some stability condition on the time step $\Delta t$. For that, we need to introduce another formulation of $\mathcal{E}^{n}$, independently of the unknowns $\widehat{\sigma}^{n+1 / 2}$.

Theorem 4.9. Considering the scheme (3.33), the corrected total discrete elastodynamic energy $\mathcal{E}^{n}$ is nonincreasing through iterations and is a positive definite quadratic form of numerical unknowns under the condition:

(1) for domains with free surface boundary conditions only, the discrete scheme (3.33) is $\mathrm{L}^{2}$-stable for all $N$ (even) if

$$
\Delta t \leq \frac{2}{\left\|M_{\Lambda}^{-1 / 2} X_{N} M_{\rho}^{-1 / 2}\right\|},
$$

(2) elsewhere, the discrete scheme (3.33) is $\mathrm{L}^{2}$-stable for $N=2$ and $\alpha \in\{1,2,3,4\}$ if

$$
\Delta t \leq \frac{2}{\left\|M_{\Lambda}^{-1 / 2} X M_{\rho}^{-1 / 2}\right\|+\left(\sum_{i=1}^{\alpha}\left|b_{i}\right|\right) \max \left(\left\|M_{\rho}^{-1 / 2} R M_{\rho}^{-1 / 2}\right\|,\left\|M_{\Lambda}^{-1 / 2} Y M_{\Lambda}^{-1 / 2}\right\|\right)},
$$

where $\|$.$\| denotes a matricial norm.$

Proof. With (3.33) in (4.1), it holds

$$
2 E_{n}=\left(\widehat{\mathbb{V}}^{n}\right)^{t} M_{\rho} \widehat{\mathbb{V}}^{n}+\left(\widehat{\sigma}^{n-\frac{1}{2}}\right)^{t}\left[M_{\Lambda} \widehat{\sigma}^{n-\frac{1}{2}}+\Delta t X_{N} \widehat{\mathbb{V}}^{n}-\Delta t Y_{N} \widehat{\sigma}^{[n]}\right]
$$

As $M_{\rho}$ and $M_{\Lambda}$ are symmetric definite positive matrices, we can bound down the discrete energy in the following way by using (3.28):

$$
\begin{aligned}
2 E_{n} \geq & \left\|M_{\rho}^{1 / 2} \widehat{\mathbb{V}}^{n}\right\|^{2}+\left\|M_{\Lambda}^{1 / 2} \widehat{\sigma}^{n-\frac{1}{2}}\right\|^{2}-\Delta t\left\|M_{\Lambda}^{1 / 2} \widehat{\sigma}^{n-\frac{1}{2}}\right\|\left\|M_{\Lambda}^{-1 / 2} X_{N} M_{\rho}^{-1 / 2}\right\|\left\|M_{\rho}^{1 / 2} \widehat{\mathbb{V}}^{n}\right\| \\
& -\Delta t \sum_{i=1}^{\alpha} b_{i}\left(\widehat{\sigma}^{n-\frac{1}{2}}\right)^{t} Y_{N} \widehat{\sigma}^{n+\frac{1}{2}-i},
\end{aligned}
$$

which gives the following estimate

$$
\begin{aligned}
2 E_{n} \geq & \left\|M_{\rho}^{1 / 2} \widehat{\mathbb{V}}^{n}\right\|^{2}\left(1-\frac{\Delta t}{2}\left\|M_{\Lambda}^{-1 / 2} X_{N} M_{\rho}^{-1 / 2}\right\|\right)+\left\|M_{\Lambda}^{1 / 2} \widehat{\sigma}^{n-\frac{1}{2}}\right\|^{2}\left(1-\frac{\Delta t}{2}\left\|M_{\Lambda}^{-1 / 2} X_{N} M_{\rho}^{-1 / 2}\right\|\right) \\
& +\frac{\Delta t}{2} \sum_{i=1}^{\alpha}\left|b_{i}\right|\left(\widehat{\sigma}^{n-\frac{1}{2}}-\gamma_{i} \widehat{\sigma}^{n+\frac{1}{2}-i}\right)^{t} Y_{N}\left(\widehat{\sigma}^{n-\frac{1}{2}}-\gamma_{i} \widehat{\sigma}^{n+\frac{1}{2}-i}\right) \\
& -\frac{\Delta t}{2} \sum_{i=1}^{\alpha}\left|b_{i}\right|\left(\widehat{\sigma}^{n-\frac{1}{2}}\right)^{t} Y_{N} \widehat{\sigma}^{n-\frac{1}{2}}-\frac{\Delta t}{2} \sum_{i=1}^{\alpha}\left|b_{i}\right|\left(\widehat{\sigma}^{n+\frac{1}{2}-i}\right)^{t} Y_{N} \widehat{\sigma}^{n+\frac{1}{2}-i} .
\end{aligned}
$$


Then, we can bound down the discrete corrected energy from (4.8)

$$
\begin{aligned}
\mathcal{E}_{n} \geq & \frac{1}{2}\left\|M_{\rho}^{1 / 2} \widehat{\mathbb{V}}^{n}\right\|^{2}\left(1-\frac{\Delta t}{2}\left\|M_{\Lambda}^{-1 / 2} X_{N} M_{\rho}^{-1 / 2}\right\|-\frac{\Delta t}{2}\left(\sum_{i=1}^{\alpha}\left|b_{i}\right|\right)\left\|M_{\rho}^{-1 / 2} R_{N} M_{\rho}^{-1 / 2}\right\|\right) \\
& +\frac{1}{2}\left\|M_{\Lambda}^{1 / 2} \widehat{\sigma}^{n-\frac{1}{2}}\right\|^{2}\left(1-\frac{\Delta t}{2}\left\|M_{\Lambda}^{-1 / 2} X_{N} M_{\rho}^{-1 / 2}\right\|-\frac{\Delta t}{2}\left(\sum_{i=1}^{\alpha}\left|b_{i}\right|\right)\left\|M_{\Lambda}^{-1 / 2} Y_{N} M_{\Lambda}^{-1 / 2}\right\|\right) \\
& +\frac{\Delta t}{4} \sum_{i=1}^{\alpha}\left|b_{i}\right|\left(\widehat{\mathbb{V}}^{n}-\gamma_{i} \widehat{\mathbb{V}}^{n+1-i}\right)^{t} R_{N}\left(\widehat{\mathbb{V}}^{n}-\gamma_{i} \widehat{\mathbb{V}}^{n+1-i}\right) \\
& +\frac{\Delta t}{4} \sum_{i=1}^{\alpha}\left|b_{i}\right|\left(\widehat{\sigma}^{n-\frac{1}{2}}-\gamma_{i} \widehat{\sigma}^{n+\frac{1}{2}-i}\right)^{t} Y_{N}\left(\widehat{\sigma}^{n-\frac{1}{2}}-\gamma_{i} \widehat{\sigma}^{n+\frac{1}{2}-i}\right) .
\end{aligned}
$$

Finally, we conclude with Remarks 3.4-3.6 and Corollaries 4.3 and 4.8 .

\section{Convergence analysis}

The objective of this section is to prove the convergence of the totally discretized scheme (3.33) following the method in $[30,56]$. We consider a family of unstructured tetrahedral meshes $\mathcal{T}_{h}$, where $h=\max _{i \in\left[1, N_{T}\right]} h_{i}$, which forms a partition of the domain $\Omega$, i.e. $\Omega=\cup_{\mathcal{T}_{i} \in \mathcal{T}_{h}} \mathcal{T}_{i}$. We assume that the unstructured meshes $\mathcal{T}_{h}$ are uniformly shape regular in the sense that there exists a constant $\xi>0$ such that

$$
\forall h>0, \quad \forall \mathcal{T}_{i} \in \mathcal{T}_{h}: \quad \frac{h_{i}}{d_{i}} \leq \xi,
$$

where $d_{i}$ is the diameter of the biggest ball included in $\mathcal{T}_{i}$. Moreover, we assume that there exists $\eta>0$ (independent of $h$ ) such that

$$
\forall h>0, \quad \forall \mathcal{T}_{i} \in \mathcal{T}_{h}, \quad \forall k \in \mathcal{V}(i): \quad \frac{h_{k}}{h_{i}} \leq \eta
$$

In what follows, we denote by

$$
\begin{aligned}
& X_{h}^{1}=\left\{\vec{\varphi}_{h} \in \mathrm{L}^{2}(\Omega)^{3}: \quad \forall i, \vec{\varphi}_{h_{\mid}} \in P_{k}\left(\mathcal{T}_{i}\right)^{3}\right\}, \\
& X_{h}^{2}=\left\{\vec{\psi}_{h} \in \mathrm{L}^{2}(\Omega)^{6}: \quad \forall i, \vec{\psi}_{h_{\left.\right|_{\mathcal{T}_{i}}}} \in P_{k}\left(\mathcal{T}_{i}\right)^{6}\right\},
\end{aligned}
$$

the sets of discontinuous piecewise polynomial fields of degree at most $k$ on each $\mathcal{T}_{i}$. We next introduce the broken Sobolev spaces

$$
P \mathrm{H}^{s}(\Omega)=\left\{v: \quad \forall i, v_{\left.\right|_{\mathcal{T}_{i}}} \in \mathrm{H}^{s}\left(\mathcal{T}_{i}\right)\right\}
$$

equipped with the norm $\|v\|_{P \mathrm{H}^{s}(\Omega)}=\left(\sum_{i=1}^{N_{T}}\left\|v_{\mid \mathcal{T}_{i}}\right\|_{\mathrm{H}^{s}\left(\mathcal{T}_{i}\right)}^{2}\right)^{1 / 2}$, where $\|\cdot\|_{\mathrm{H}^{s}\left(\mathcal{T}_{i}\right)}$ denotes the standard $\mathrm{H}^{s}$-norm on $\mathcal{T}_{i}$.

\subsection{Definition and properties of the semi-discretized scheme}

First, we are interested in the study of consistency and stability of the spatially semi-discretized problem below. In what follows, we set $\vec{\varphi}_{i}$ (resp. $\vec{\psi}_{i}$ ) the restriction of $\vec{\varphi} \in \mathrm{H}^{s}\left(\mathcal{T}_{h}\right)^{3}$ (resp. $\vec{\psi} \in \mathrm{H}^{s}\left(\mathcal{T}_{h}\right)^{6}$ ) to the element $\mathcal{T}_{i}$ with $s>\frac{1}{2}$. The semi-discrete solution $\left(\vec{V}_{h}, \overrightarrow{\tilde{\sigma}}_{h}\right)$ defined in $\mathrm{C}^{1}\left([0, T], X_{h}^{1} \times X_{h}^{2}\right)$ is the solution of the weak 
formulation (3.16) and (3.17), for any test field $\left(\vec{\varphi}_{h}, \vec{\psi}_{h}\right) \in X_{h}^{1} \times X_{h}^{2}$, for $0 \leq t \leq T$ and all $i$, with the initial values

$$
\vec{V}_{h}(0)=P_{h}^{1}\left(\vec{V}_{0}\right) \text { and } \quad \overrightarrow{\tilde{\sigma}}_{h}(0)=P_{h}^{2}\left(\overrightarrow{\tilde{\sigma}}_{0}\right),
$$

where $P_{h}^{1}: \mathrm{L}^{2}(\Omega)^{3} \rightarrow X_{h}^{1}$ and $P_{h}^{2}: \mathrm{L}^{2}(\Omega)^{6} \rightarrow X_{h}^{2}$ denote respectively the orthogonal projection onto $X_{h}^{1}$ and $X_{h}^{2}$.

Definition 5.1. For given vector fields $\vec{W}=(\vec{V}, \overrightarrow{\tilde{\sigma}})$ and $\vec{W}^{\prime}=\left(\vec{V}^{\prime}, \overrightarrow{\tilde{\sigma}}^{\prime}\right)$, we introduce the following bilinear forms which are well defined on $X_{h}^{1} \times X_{h}^{2}$ :

$$
\begin{aligned}
m\left(\vec{W}, \vec{W}^{\prime}\right)= & \sum_{i=1}^{N_{T}} \int_{\mathcal{T}_{i}}\left[\rho_{i}\left(\vec{V}_{i}^{\prime}\right)^{t} \vec{V}_{i}+\left(\overrightarrow{\tilde{\sigma}}_{i}^{\prime}\right)^{t} \Lambda_{i} \overrightarrow{\tilde{\sigma}}_{i}\right] \mathrm{d} v \\
a\left(\vec{W}, \vec{W}^{\prime}\right)= & \sum_{i=1}^{N_{T}} \int_{\mathcal{T}_{i}} \sum_{\alpha \in\{x, y, z\}}\left[\left(\partial_{\alpha} \vec{V}_{i}^{\prime}\right)^{t} \mathbb{M}_{\alpha} \overrightarrow{\tilde{\sigma}}_{i}+\left(\partial_{\alpha} \overrightarrow{\tilde{\sigma}}_{i}^{\prime}\right)^{t} \mathbb{M}_{\alpha}^{t} \vec{V}_{i}\right] \mathrm{d} v \\
b\left(\vec{W}, \vec{W}^{\prime}\right)= & -\sum_{i=1}^{N_{T}} \sum_{k \in \mathcal{V}(i)} \int_{S_{i k}}\left[\left(\vec{V}_{i}^{\prime}\right)^{t} \mathbb{P}_{i k} \frac{\overrightarrow{\tilde{\sigma}}_{i}+\overrightarrow{\tilde{\sigma}}_{k}}{2}+\left(\overrightarrow{\tilde{\sigma}}_{i}^{\prime}\right)^{t} \mathbb{P}_{i k}^{t} \frac{\vec{V}_{i}+\vec{V}_{k}}{2}\right] \mathrm{d} s \\
& -\frac{1}{2} \sum_{i=1}^{N_{T}} \sum_{k \in \mathcal{E}(i)} \int_{S_{i k}}\left[\left(\vec{V}_{i}^{\prime}\right)^{t}\left(\mathbb{P}_{i k} \overrightarrow{\tilde{\sigma}}_{i}-\mathbb{A}_{i k} \vec{V}_{i}\right)+\left(\overrightarrow{\tilde{\sigma}}_{i}^{\prime}\right)^{t}\left(\mathbb{P}_{i k}^{t} \vec{V}_{i}-\mathbb{B}_{i k} \overrightarrow{\tilde{\sigma}}_{i}\right)\right] \mathrm{d} s \\
& -\sum_{i=1}^{N_{T}} \sum_{k \in \mathcal{K}(i)} \int_{S_{i k}}\left(\overrightarrow{\tilde{\sigma}}_{i}^{\prime}\right)^{t} \mathbb{P}_{i k}^{t} \vec{V}_{i} \mathrm{~d} s .
\end{aligned}
$$

Thanks to (3.16) and (3.17), we remark the following result:

Proposition 5.2. The semi-discrete solution $\vec{W}_{h}=\left(\vec{V}_{h}, \overrightarrow{\tilde{\sigma}}_{h}\right)$ of (3.16), (3.17) and (5.6) satisfies

$$
m\left(\partial_{t} \vec{W}_{h}, \vec{W}_{h}^{\prime}\right)+a\left(\vec{W}_{h}, \vec{W}_{h}^{\prime}\right)+b\left(\vec{W}_{h}, \vec{W}_{h}^{\prime}\right)=0, \forall \vec{W}_{h}^{\prime} \in X_{h}^{1} \times X_{h}^{2} .
$$

Proposition 5.3. The exact solution $\vec{W}=(\vec{V}, \overrightarrow{\tilde{\sigma}})$ of (2.6)-(2.9) satisfies the following property

$$
m\left(\partial_{t} \vec{W}, \vec{W}_{h}^{\prime}\right)+a\left(\vec{W}, \vec{W}_{h}^{\prime}\right)+b\left(\vec{W}, \vec{W}_{h}^{\prime}\right)=0, \forall \vec{W}_{h}^{\prime} \in X_{h}^{1} \times X_{h}^{2} .
$$

Proof. According to $(3.1 \mathrm{a})-(3.1 \mathrm{~b})$, we have to verify that the quantity $b\left(\vec{W}, \vec{W}_{h}^{\prime}\right)$ is equal to

$$
-\sum_{i=1}^{N_{T}} \sum_{\alpha \in\{x, y, z\}} \int_{\partial \mathcal{T}_{i}}\left[\left(\vec{V}_{i}^{\prime}\right)^{t} \mathbb{M}_{\alpha} \overrightarrow{\tilde{\sigma}} n_{\alpha_{i}}+\left(\overrightarrow{\tilde{\sigma}}_{i}^{\prime}\right)^{t} \mathbb{M}_{\alpha}^{t} \vec{V} n_{\alpha_{i}}\right] \mathrm{d} s
$$

The free surface boundary condition (2.7) results in $\sum_{i=1}^{N_{T}} \sum_{k \in \mathcal{K}(i)} \int_{S_{i k}}\left(\vec{V}_{i}^{\prime}\right)^{t} \mathbb{P}_{i k} \overrightarrow{\tilde{\sigma}} \mathrm{d} s=0$. On the other hand, thanks to the absorbing boundary conditions (2.8), we also have:

$$
\frac{1}{2} \sum_{i=1}^{N_{T}} \sum_{k \in \mathcal{E}(i)} \int_{S_{i k}}\left[\left(\vec{V}_{i}^{\prime}\right)^{t}\left(\mathbb{P}_{i k} \overrightarrow{\tilde{\sigma}}-\mathbb{A}_{i k} \vec{V}\right)+\left(\overrightarrow{\tilde{\sigma}}_{i}^{\prime}\right)^{t}\left(\mathbb{P}_{i k}^{t} \vec{V}-\mathbb{B}_{i k} \overrightarrow{\tilde{\sigma}}\right)\right] \mathrm{d} s=\sum_{i=1}^{N_{T}} \sum_{k \in \mathcal{E}(i)} \int_{S_{i k}}\left[\left(\vec{V}_{i}^{\prime}\right)^{t} \mathbb{P}_{i k} \overrightarrow{\tilde{\sigma}}+\left(\overrightarrow{\tilde{\sigma}}_{i}^{\prime}\right)^{t} \mathbb{P}_{i k}^{t} \vec{V}\right] \mathrm{d} s
$$

which closes the proof. 
Lemma 5.4. For any $\vec{W}_{h}=\left(\vec{\varphi}_{h}, \vec{\psi}_{h}\right) \in X_{h}^{1} \times X_{h}^{2}$, the following quantity is positive:

$$
a\left(\vec{W}_{h}, \vec{W}_{h}\right)+b\left(\vec{W}_{h}, \vec{W}_{h}\right)=\frac{1}{2} \sum_{i=1}^{N_{T}} \sum_{k \in \mathcal{E}(i)} \int_{S_{i k}}\left[\vec{\varphi}_{i}^{t} \mathbb{A}_{i k} \vec{\varphi}_{i}+\vec{\psi}_{i}^{t} \mathbb{B}_{i k} \vec{\psi}_{i}\right] \mathrm{d} s \geq 0 .
$$

Proof. Let be $\vec{W}_{h}=\left(\vec{\varphi}_{h}, \vec{\psi}_{h}\right) \in X_{h}^{1} \times X_{h}^{2}$ a given vector field. Then, we get

$$
\begin{aligned}
a\left(\vec{W}_{h}, \vec{W}_{h}\right)+b\left(\vec{W}_{h}, \vec{W}_{h}\right)= & {\left[\sum_{i=1}^{N_{T}} \sum_{\alpha \in\{x, y, z\}} \int_{\mathcal{T}_{i}}\left[\left(\partial_{\alpha} \vec{\varphi}_{i}\right)^{t} \mathbb{M}_{\alpha} \vec{\psi}_{i}+\left(\partial_{\alpha} \vec{\psi}_{i}\right)^{t} \mathbb{M}_{\alpha}^{t} \vec{\varphi}_{i}\right] \mathrm{d} v\right.} \\
& -\frac{1}{2} \sum_{i=1}^{N_{T}} \sum_{k \in \mathcal{V}(i)} \int_{S_{i k}}\left[\vec{\varphi}_{i}^{t} \mathbb{P}_{i k} \vec{\psi}_{i}+\vec{\psi}_{i}^{t} \mathbb{P}_{i k}^{t} \vec{\varphi}_{i}\right] \mathrm{d} s-\sum_{i=1}^{N_{T}} \sum_{k \in \mathcal{K}(i)} \int_{S_{i k}} \vec{\psi}_{i}^{t} \mathbb{P}_{i k}^{t} \vec{\varphi}_{i} \mathrm{~d} s \\
& \left.-\frac{1}{2} \sum_{i=1}^{N_{T}} \sum_{k \in \mathcal{E}(i)} \int_{S_{i k}}\left[\vec{\varphi}_{i}^{t} \mathbb{P}_{i k} \vec{\psi}_{i}+\vec{\psi}_{i}^{t} \mathbb{P}_{i k}^{t} \vec{\varphi}_{i}\right] \mathrm{d} s\right] \\
& -\frac{1}{2} \sum_{i=1}^{N_{T}} \sum_{k \in \mathcal{V}(i)} \int_{S_{i k}}\left[\vec{\varphi}_{i}^{t} \mathbb{P}_{i k} \vec{\psi}_{k}+\vec{\psi}_{i}^{t} \mathbb{P}_{i k}^{t} \vec{\varphi}_{k}\right] \mathrm{d} s \\
& +\frac{1}{2} \sum_{i=1}^{N_{T}} \sum_{k \in \mathcal{E}(i)} \int_{S_{i k}}\left[\vec{\varphi}_{i}^{t} \mathbb{A}_{i k} \vec{\varphi}_{i}+\vec{\psi}_{i}^{t} \mathbb{B}_{i k} \vec{\psi}_{i}\right] \mathrm{d} s .
\end{aligned}
$$

The first term (between brackets) vanishes by the Green Formula:

$$
\int_{\mathcal{T}_{i}}\left[\left(\partial_{\alpha} \vec{\varphi}_{i}\right)^{t} \mathbb{M}_{\alpha} \vec{\psi}_{i}+\left(\partial_{\alpha} \vec{\psi}_{i}\right)^{t} \mathbb{M}_{\alpha}^{t} \vec{\varphi}_{i}\right] \mathrm{d} v=\int_{\partial \mathcal{T}_{i}} \vec{\varphi}_{i}^{t} \mathbb{M}_{\alpha} n_{\alpha_{i}} \vec{\psi}_{i} \mathrm{~d} s
$$

combined to the equality $\vec{\psi}_{i}^{t} \mathbb{P}_{i k}^{t} \vec{\varphi}_{i}=\vec{\varphi}_{i}^{t} \mathbb{P}_{i k} \vec{\psi}_{i}$. Moreover, since $\mathbb{P}_{i k}=\sum_{\alpha \in\{x, y, z\}} \mathbb{M}_{\alpha} n_{\alpha_{i k}}$ and thanks to the orientation of $\overrightarrow{\mathbf{n}}_{i k}$ from $\mathcal{T}_{i}$ to $\mathcal{T}_{k}$, we remark that:

$$
\sum_{i=1}^{N_{T}} \sum_{k \in \mathcal{V}(i)} \int_{S_{i k}} \vec{\psi}_{i}^{t} \mathbb{P}_{i k}^{t} \vec{\varphi}_{k} \mathrm{~d} s=-\sum_{i=1}^{N_{T}} \sum_{k \in \mathcal{V}(i)} \int_{S_{i k}} \vec{\varphi}_{i}^{t} \mathbb{P}_{i k} \vec{\psi}_{k} \mathrm{~d} s,
$$

which implies that the second term is also equal to zero. Therefore, it remains only the third term depending on $\mathbb{A}_{i k}$ and $\mathbb{B}_{i k}$, which are respectively a real symmetric definite positive matrix and a real symmetric semi-definite positive matrix.

Consequently, if we define the semi-discrete energy by

$$
\mathcal{E}_{h}(t)=\frac{1}{2} m\left(\vec{W}_{h}(t), \vec{W}_{h}(t)\right),
$$

where $\vec{W}_{h}(t)=\left(\vec{V}_{h}(t), \overrightarrow{\tilde{\sigma}}_{h}(t)\right)$ is the semi-discrete solution of (3.16), (3.17) and (5.6), we deduce from Proposition 5.2 and Lemma 5.4 that

$$
\partial_{t} \mathcal{E}_{h}(t) \leq 0, \quad \forall t \in[0, T],
$$

which implies that $\mathcal{E}_{h}(t)$ is decreasing on $[0, T]$ and we have

$$
\mathcal{E}_{h}(t) \leq \mathcal{E}_{h}(0) \leq E(0), \quad \forall t \in[0, T]
$$




\subsection{Convergence of the semi-discretized problem}

We shall at first give a convergence result for the spatially semi-discretized scheme (3.16), (3.17) and (5.6). We recall the two following lemmae, well-known in the framework of the finite elements (see [17]):

Lemma 5.5. Let $\mathcal{T}_{i} \in \mathcal{T}_{h}$, we assume that $u$ belongs to the space $\mathrm{H}^{s+1}\left(\mathcal{T}_{i}\right)$ for $s \geq 0$. Let $\Pi$ be a linear continuous operator from $\mathrm{H}^{s+1}\left(\mathcal{T}_{i}\right)$ onto $P_{k}\left(\mathcal{T}_{i}\right)$ such that $\Pi(u)=u$ for all $u \in P_{k}\left(\mathcal{T}_{i}\right)$. Then we have

$$
\begin{aligned}
\|u-\Pi(u)\|_{\mathrm{L}^{2}\left(T_{i}\right)} & \leq C_{1} h_{i}^{\min \{s, k\}+1}\|u\|_{\mathrm{H}^{s+1}\left(\mathcal{T}_{i}\right)}, \\
\|u-\Pi(u)\|_{\mathrm{L}^{2}\left(\partial \mathcal{T}_{i}\right)} & \leq C_{1} h_{i}^{\min \{s, k\}+1 / 2}\|u\|_{\mathrm{H}^{s+1}\left(\mathcal{T}_{i}\right)},
\end{aligned}
$$

where $C_{1}$ is a positive constant only depending on $k, s$ and the regularity parameter $\xi$ of the mesh.

Lemma 5.6. For all $p \in P_{k}\left(\mathcal{T}_{i}\right)$, we have

$$
\|p\|_{\mathrm{L}^{2}\left(\partial \mathcal{T}_{i}\right)} \leq C_{2} h_{i}^{-1 / 2}\|p\|_{\mathrm{L}^{2}\left(\mathcal{T}_{i}\right)},
$$

where $C_{2}$ is a positive constant only depending on $k$ and the regularity parameter $\xi$ of the mesh.

Thus, we can state the following Lemma:

Lemma 5.7. Let be $\vec{W}=(\vec{V}, \overrightarrow{\tilde{\sigma}})$ the exact solution of (2.6)-(2.9) supposed to belong to $\mathrm{C}^{0}\left([0, T], P \mathrm{H}^{s+1}(\Omega)^{9}\right)$ and $\left(\vec{V}_{h}, \overrightarrow{\tilde{\sigma}}_{h}\right) \in \mathrm{C}^{1}\left([0, T], X_{h}^{1} \times X_{h}^{2}\right)$ be the solution of the semi-discrete problem (3.16), (3.17) and (5.6). For any $t \in[0, T]$, we have

$$
\begin{aligned}
& b\left(\vec{W}-P_{h}^{0}(\vec{W}), \vec{W}_{h}-P_{h}^{0}(\vec{W})\right)(t) \\
\leq & K h^{\min \{s, k\}}\left[\left\|\left(\vec{V}_{h}-P_{h}^{1}(\vec{V})\right)(t)\right\|_{L^{2}(\Omega)^{3}}^{2}+\left\|\left(\overrightarrow{\tilde{\sigma}}_{h}-P_{h}^{2}(\overrightarrow{\tilde{\sigma}})\right)(t)\right\|_{\mathrm{L}^{2}(\Omega)^{6}}^{2}\right]^{1 / 2}\|(\vec{V}, \overrightarrow{\tilde{\sigma}})(t)\|_{P \mathrm{H}^{s+1}(\Omega)^{9}} .
\end{aligned}
$$

Proof. The two previous Lemmae 5.5 and 5.6 imply together the following estimates:

$$
\begin{aligned}
& \int_{S_{i l}}\left(\vec{V}_{h}-P_{h}^{1}(\vec{V})\right)_{\left.\right|_{\mathcal{T}_{i}}} \mathbb{P}_{i l}\left(\overrightarrow{\tilde{\sigma}}-P_{h}^{2}(\overrightarrow{\tilde{\sigma}})\right)_{\left.\right|_{\mathcal{T}_{i}}} \mathrm{~d} s \leq C\left\|\mathbb{P}_{i l}\right\| h_{i}^{\min \{s, k\}}\left\|\vec{V}_{h}-P_{h}^{1}(\vec{V})\right\|_{\mathrm{L}^{2}\left(\mathcal{T}_{i}\right)^{3}}\|\overrightarrow{\tilde{\sigma}}\|_{\mathrm{H}^{s+1}\left(\mathcal{T}_{i}\right)^{6}} \\
& \int_{S_{i l}}\left(\overrightarrow{\tilde{\sigma}}_{h}-P_{h}^{2}(\overrightarrow{\tilde{\sigma}})\right)_{\left.\right|_{\mathcal{T}_{i}}} \mathbb{P}_{i l}^{t}\left(\vec{V}-P_{h}^{1}(\vec{V})\right)_{\mid \mathcal{T}_{i}} \mathrm{~d} s \leq C\left\|\mathbb{P}_{i l}\right\| h_{i}^{\min \{s, k\}}\left\|\overrightarrow{\tilde{\sigma}}_{h}-P_{h}^{2}(\overrightarrow{\tilde{\sigma}})\right\|_{\mathrm{L}^{2}\left(\mathcal{T}_{i}\right)^{6}}\|\vec{V}\|_{\mathrm{H}^{s+1}\left(\mathcal{T}_{i}\right)^{3}}, \\
& \int_{S_{i l}}\left(\vec{V}_{h}-P_{h}^{1}(\vec{V})\right)_{\mid \mathcal{T}_{i}} \mathbb{A}_{i l}\left(\vec{V}-P_{h}^{1}(\vec{V})\right)_{\left.\right|_{\mathcal{T}_{i}}} \mathrm{~d} s \leq C r_{+}\left(\mathbb{A}_{i l}\right) h_{i}^{\min \{s, k\}}\left\|\vec{V}_{h}-P_{h}^{1}(\vec{V})\right\|_{\mathrm{L}^{2}\left(\mathcal{T}_{i}\right)^{3}}\|\vec{V}\|_{\mathrm{H}^{s+1}\left(\mathcal{T}_{i}\right)^{3}}, \\
& \int_{S_{i l}}\left(\overrightarrow{\tilde{\sigma}}_{h}-P_{h}^{2}(\overrightarrow{\tilde{\sigma}})\right)_{\left.\right|_{\mathcal{I}_{i}}} \mathbb{B}_{i l}\left(\overrightarrow{\tilde{\sigma}}-P_{h}^{2}(\overrightarrow{\tilde{\sigma}})\right)_{\left.\right|_{\mathcal{T}_{i}}} \mathrm{~d} s \leq C r_{+}\left(\mathbb{B}_{i l}\right) h_{i}^{\min \{s, k\}}\left\|\overrightarrow{\tilde{\sigma}}_{h}-P_{h}^{2}(\overrightarrow{\tilde{\sigma}})\right\|_{\mathrm{L}^{2}\left(\mathcal{T}_{i}\right)^{6}}\|\overrightarrow{\tilde{\sigma}}\|_{\mathrm{H}^{s+1}\left(\mathcal{T}_{i}\right)^{6}},
\end{aligned}
$$

where $C=C_{1} C_{2}, l \in \mathcal{V}(i) \cup \mathcal{E}(i)$ in (5.22), $l \in \mathcal{V}(i) \cup \mathcal{K}(i) \cup \mathcal{E}(i)$ in (5.23), $l \in \mathcal{E}(i)$ in (5.24) and (5.25). In addition, applying (5.2), we have in the same way, for all $l \in \mathcal{V}(i)$ :

$$
\begin{aligned}
& \int_{S_{i l}}\left(\vec{V}_{h}-P_{h}^{1}(\vec{V})\right)_{\left.\right|_{\mathcal{T}_{i}}} \mathbb{P}_{i l}\left(\overrightarrow{\tilde{\sigma}}-P_{h}^{2}(\overrightarrow{\tilde{\sigma}})\right)_{\mid \mathcal{I}_{l}} \mathrm{~d} s \\
& \leq C\left\|\mathbb{P}_{i l}\right\| \eta^{\min \{s, k\}+1 / 2} h_{i}^{\min \{s, k\}}\left\|\vec{V}_{h}-P_{h}^{1}(\vec{V})\right\|_{\mathrm{L}^{2}\left(\mathcal{T}_{i}\right)^{3}}\|\overrightarrow{\tilde{\sigma}}\|_{\mathrm{H}^{s+1}\left(\mathcal{T}_{l}\right)^{6}}, \\
& \int_{S_{i l}}\left(\overrightarrow{\tilde{\sigma}}_{h}-P_{h}^{2}(\overrightarrow{\tilde{\sigma}})\right)_{\left.\right|_{\tau_{i}}} \mathbb{P}_{i l}^{t}\left(\vec{V}-P_{h}^{1}(\vec{V})\right)_{\left.\right|_{\tau_{l}}} \mathrm{~d} s \\
& \leq C\left\|\mathbb{P}_{i l}\right\| \eta^{\min \{s, k\}+1 / 2} h_{i}^{\min \{s, k\}}\left\|\overrightarrow{\tilde{\sigma}}_{h}-P_{h}^{2}(\overrightarrow{\tilde{\sigma}})\right\|_{\mathrm{L}^{2}\left(\mathcal{T}_{i}\right)^{6}}\|\vec{V}\|_{\mathrm{H}^{s+1}\left(\mathcal{T}_{l}\right)^{3}} .
\end{aligned}
$$


Theorem 5.8. Let $\left(\mathcal{T}_{h}\right)_{h}$ be a family of unstructured meshes satisfying (5.1) and (5.2).

Let $(\vec{V}, \overrightarrow{\tilde{\sigma}})$ be the exact solution of the symmetric pseudo-conservative problem (2.6)-(2.9) supposed to belong to $\mathrm{C}^{0}\left([0, T] ; P \mathrm{H}^{s+1}(\Omega)^{9}\right)$ for $s \geq 0$, and let $\left(\vec{V}_{h}, \overrightarrow{\tilde{\sigma}}_{h}\right) \in \mathrm{C}^{1}\left([0, T], X_{h}^{1} \times X_{h}^{2}\right)$ be the solution of the semi-discrete problem (3.16), (3.17) and (5.6). Then, there exists a constant $K>0$ independent of $h$ such that

$$
\max _{t \in[0, T]}\left[\left\|\left(\vec{V}-\vec{V}_{h}\right)(t)\right\|_{\mathrm{L}^{2}(\Omega)^{3}}^{2}+\left\|\left(\overrightarrow{\tilde{\sigma}}-\overrightarrow{\tilde{\sigma}}_{h}\right)(t)\right\|_{\mathrm{L}^{2}(\Omega)^{6}}^{2}\right]^{1 / 2} \leq K T h^{\min \{s, k\}}\|(\vec{V}, \overrightarrow{\tilde{\sigma}})\|_{\mathrm{C}^{0}\left([0, T], P \mathrm{H}^{s+1}(\Omega)^{9}\right)} .
$$

Proof. First, applying Lemma 5.4 to the vector $\vec{W}_{h}-P_{h}^{0}(\vec{W})=\left(\vec{V}_{h}-P_{h}^{1}(\vec{V}), \overrightarrow{\tilde{\sigma}}_{h}-P_{h}^{2}(\overrightarrow{\tilde{\sigma}})\right) \in X_{h}^{1} \times X_{h}^{2}$, we get

$$
a\left(\vec{W}_{h}-P_{h}^{0}(\vec{W}), \vec{W}_{h}-P_{h}^{0}(\vec{W})\right)+b\left(\vec{W}_{h}-P_{h}^{0}(\vec{W}), \vec{W}_{h}-P_{h}^{0}(\vec{W})\right) \geq 0,
$$

and, then

$$
\begin{aligned}
m\left(\partial_{t}\left(\vec{W}_{h}-P_{h}^{0}(\vec{W})\right), \vec{W}_{h}-P_{h}^{0}(\vec{W})\right) \leq & m\left(\partial_{t}\left(\vec{W}_{h}-P_{h}^{0}(\vec{W})\right), \vec{W}_{h}-P_{h}^{0}(\vec{W})\right) \\
& +a\left(\vec{W}_{h}-P_{h}^{0}(\vec{W}), \vec{W}_{h}-P_{h}^{0}(\vec{W})\right) \\
& +b\left(\vec{W}_{h}-P_{h}^{0}(\vec{W}), \vec{W}_{h}-P_{h}^{0}(\vec{W})\right) .
\end{aligned}
$$

Thus, since $\vec{W}_{h}-P_{h}^{0}(\vec{W}) \in X_{h}^{1} \times X_{h}^{2}$, the difference between (5.10) and (5.11) gives us

$$
m\left(\partial_{t}\left(\vec{W}_{h}-\vec{W}\right), \vec{W}_{h}-P_{h}^{0}(\vec{W})\right)+a\left(\vec{W}_{h}-\vec{W}, \vec{W}_{h}-P_{h}^{0}(\vec{W})\right)+b\left(\vec{W}_{h}-\vec{W}, \vec{W}_{h}-P_{h}^{0}(\vec{W})\right)=0 .
$$

After, we substract the previous equality in the right-hand side of (5.27) and we obtain

$$
\begin{aligned}
m\left(\partial_{t}\left(\vec{W}_{h}-P_{h}^{0}(\vec{W})\right), \vec{W}_{h}-P_{h}^{0}(\vec{W})\right) \leq & m\left(\partial_{t}\left(\vec{W}-P_{h}^{0}(\vec{W})\right), \vec{W}_{h}-P_{h}^{0}(\vec{W})\right) \\
& +a\left(\vec{W}-P_{h}^{0}(\vec{W}), \vec{W}_{h}-P_{h}^{0}(\vec{W})\right) \\
& +b\left(\vec{W}-P_{h}^{0}(\vec{W}), \vec{W}_{h}-P_{h}^{0}(\vec{W})\right) .
\end{aligned}
$$

As $P_{h}^{1}(\vec{V})\left(\operatorname{resp} . P_{h}^{2}(\overrightarrow{\tilde{\sigma}})\right)$ is the orthogonal projection of $\vec{V}$ (resp. $\overrightarrow{\tilde{\sigma}}$ ) onto $X_{h}^{1}$ (resp. $\left.X_{h}^{2}\right)$ and $\vec{W}_{h}-P_{h}^{0}(\vec{W}) \in$ $X_{h}^{1} \times X_{h}^{2}$, we necessarily have

$$
a\left(\vec{W}-P_{h}^{0}(\vec{W}), \vec{W}_{h}-P_{h}^{0}(\vec{W})\right)=0
$$

and

$$
m\left(\partial_{t}\left(\vec{W}-P_{h}^{0}(\vec{W})\right), \vec{W}_{h}-P_{h}^{0}(\vec{W})\right)=\frac{1}{2} \frac{\mathrm{d}}{\mathrm{d} t} m\left(\left(\vec{W}-P_{h}^{0}(\vec{W})\right), \vec{W}_{h}-P_{h}^{0}(\vec{W})\right)=0 .
$$

On the other hand, we can check that

$$
\begin{aligned}
& m\left(\partial_{t}\left(\vec{W}_{h}-P_{h}^{0}(\vec{W})\right), \vec{W}_{h}-P_{h}^{0}(\vec{W})\right) \\
= & \frac{1}{2} \sum_{i=1}^{N_{T}} \frac{\mathrm{d}}{\mathrm{d} t} \int_{T_{i}}\left[\rho_{i}\left|\vec{V}_{h}-P_{h}^{1}(\vec{V})\right|^{2}(t)+\left(\left(\overrightarrow{\tilde{\sigma}}_{h}-P_{h}^{2}(\overrightarrow{\tilde{\sigma}})\right) \Lambda_{i}\left(\overrightarrow{\tilde{\sigma}}_{h}-P_{h}^{2}(\overrightarrow{\tilde{\sigma}})\right)\right)(t)\right] \mathrm{d} v
\end{aligned}
$$

so, by taking also into account (5.29) and (5.30) in (5.28), we obtain

$$
\begin{aligned}
& \frac{\mathrm{d}}{\mathrm{d} t}\left[\left\|\left(\vec{V}_{h}-P_{h}^{1}(\vec{V})\right)(t)\right\|_{\mathrm{L}^{2}(\Omega)^{3}}^{2}+\left\|\left(\overrightarrow{\tilde{\sigma}}_{h}-P_{h}^{2}(\overrightarrow{\tilde{\sigma}})\right)(t)\right\|_{\mathrm{L}^{2}(\Omega)^{6}}^{2}\right] \mathrm{d} v \\
\leq & 2\left(\min _{1 \leq i \leq N_{T}}\left\{\rho_{i}, r_{-}\left(\Lambda_{i}\right)\right\}\right)^{-1} b\left(\vec{W}-P_{h}^{0}(\vec{W}), \vec{W}_{h}-P_{h}^{0}(\vec{W})\right)(t) .
\end{aligned}
$$

Then, we integrate (5.32) from 0 to $t \in[0, T]$ and, since (2.9) and (5.6) hold, we have

$$
\begin{aligned}
& \left\|\left(\vec{V}_{h}-P_{h}^{1}(\vec{V})\right)(t)\right\|_{\mathrm{L}^{2}(\Omega)^{3}}^{2}+\left\|\left(\overrightarrow{\tilde{\sigma}}_{h}-P_{h}^{2}(\overrightarrow{\tilde{\sigma}})\right)(t)\right\|_{\mathrm{L}^{2}(\Omega)^{6}}^{2} \\
\leq & 2\left(\min _{1 \leq i \leq N_{T}}\left\{\rho_{i}, r_{-}\left(\Lambda_{i}\right)\right\}\right)^{-1} \int_{0}^{T} b\left(\vec{W}-P_{h}^{0}(\vec{W}), \vec{W}_{h}-P_{h}^{0}(\vec{W})\right)(t) \mathrm{d} t .
\end{aligned}
$$


Therefore, the last inequality becomes

$$
\begin{aligned}
& \max _{t \in[0, T]}\left[\left\|\left(\vec{V}_{h}-P_{h}^{1}(\vec{V})\right)(t)\right\|_{L^{2}(\Omega)^{3}}^{2}+\left\|\left(\overrightarrow{\tilde{\sigma}}_{h}-P_{h}^{2}(\overrightarrow{\tilde{\sigma}})\right)(t)\right\|_{L^{2}(\Omega)^{6}}^{2}\right] \\
\leq & 2\left(\min _{1 \leq i \leq N_{T}}\left\{\rho_{i}, r_{-}\left(\Lambda_{i}\right)\right\}\right)^{-1} \int_{0}^{T} b\left(\vec{W}-P_{h}^{0}(\vec{W}), \vec{W}_{h}-P_{h}^{0}(\vec{W})\right)(t) \mathrm{d} t
\end{aligned}
$$

which implies, by Lemma 5.7, that there exists $K>0$ such that

$$
\max _{t \in[0, T]}\left[\left\|\left(\vec{V}_{h}-P_{h}^{1}(\vec{V})\right)(t)\right\|_{\mathrm{L}^{2}(\Omega)^{3}}^{2}+\left\|\left(\overrightarrow{\tilde{\sigma}}_{h}-P_{h}^{2}(\overrightarrow{\tilde{\sigma}})\right)(t)\right\|_{\mathrm{L}^{2}(\Omega)^{6}}^{2}\right]^{1 / 2} \leq K T h^{\min \{s, k\}}\|(\vec{V}, \overrightarrow{\tilde{\sigma}})\|_{\mathrm{C}^{0}\left([0, T], P \mathrm{H}^{s+1}(\Omega)^{9}\right)} .
$$

On the other hand, we also have by Lemma 5.5 the existence of $C>0$ such that: $\forall t \in[0, T]$,

$\left[\left\|\left(\vec{V}-P_{h}^{1}(\vec{V})\right)(t)\right\|_{\mathrm{L}^{2}(\Omega)^{3}}^{2}+\left\|\left(\overrightarrow{\tilde{\sigma}}-P_{h}^{2}(\overrightarrow{\tilde{\sigma}})\right)(t)\right\|_{\mathrm{L}^{2}(\Omega)^{6}}^{2}\right]^{1 / 2} \leq C h^{\min \{s, k\}+1}\left(\|\vec{V}(t)\|_{P \mathrm{H}^{s+1}(\Omega)^{3}}^{2}+\|\overrightarrow{\tilde{\sigma}}(t)\|_{P \mathrm{H}^{s+1}(\Omega)^{6}}^{2}\right)^{1 / 2}$,

which combined to (5.36) concludes the proof.

\subsection{Convergence of the totally discretized problem}

In this section, we prove the convergence of the totally discretized scheme (3.33), following the method proposed in [56] for the second order leap-frog scheme.

Theorem 5.9. Let $\left(\vec{V}_{h}^{n}, \overrightarrow{\tilde{\sigma}}_{h}^{n+1 / 2}\right)$ be the solution associated with (3.33) according to (3.2) and (3.3).

(1) Let $\left(\vec{V}_{h}, \overrightarrow{\tilde{\sigma}}_{h}\right) \in \mathrm{C}^{N+1}\left([0, T], X_{h}^{1} \times X_{h}^{2}\right)$ be the solution of the semi-discrete problem (3.16), (3.17) and (5.6), and let $(\vec{V}, \overrightarrow{\tilde{\sigma}}) \in \mathrm{C}^{N+1}\left([0, T], \mathrm{L}^{2}(\Omega)^{9}\right) \cap \mathrm{C}^{0}\left([0, T], P \mathrm{H}^{s+1}(\Omega)^{9}\right)$ be the exact solution of $(2.6)-(2.9)$. Under a condition on the time step $\Delta t$ of the same type as (4.19), there exists a constant $C>0$ such that the following error estimate holds:

$$
\begin{aligned}
& \max _{n=0, \ldots, N}\left(\left\|\vec{V}\left(t_{n}\right)-\vec{V}_{h}^{n}\right\|_{\mathrm{C}^{0}\left([0, T], \mathrm{L}^{2}(\Omega)^{3}\right)}^{2}+\left\|\overrightarrow{\tilde{\sigma}}\left(t_{n+\frac{1}{2}}\right)-\overrightarrow{\tilde{\sigma}}_{h}^{n+\frac{1}{2}}\right\|_{\mathrm{C}^{0}\left([0, T], \mathrm{L}^{2}(\Omega)^{6}\right)}^{2}\right)^{1 / 2} \\
\leq & C \Delta t^{N}\|(\vec{V}, \overrightarrow{\tilde{\sigma}})\|_{\mathrm{C}^{N+1}\left([0, T], \mathrm{L}^{2}(\Omega)^{9}\right)}+C h^{\min (s, k)}\|(\vec{V}, \overrightarrow{\tilde{\sigma}})\|_{\mathrm{C}^{0}\left([0, T], P \mathrm{H}^{s+1}(\Omega)^{9}\right) .}
\end{aligned}
$$

(2) Let $\left(\vec{V}_{h}, \overrightarrow{\tilde{\sigma}}_{h}\right) \in \mathrm{C}^{\max (\alpha, 3)}\left([0, T], X_{h}^{1} \times X_{h}^{2}\right)$ be the solution of the semi-discrete problem (3.16) and (3.17) and (5.6), where $\alpha \in\{1,2,3,4\}$ is the coefficient defined at (3.28). Let $(\vec{V}, \overrightarrow{\tilde{\sigma}}) \in \mathrm{C}^{\max (\alpha, 3)}\left([0, T], \mathrm{L}^{2}(\Omega)^{9}\right) \cap$ $\mathrm{C}^{0}\left([0, T], P \mathrm{H}^{s+1}(\Omega)^{9}\right)$ be the exact solution of $(2.6)-(2.9)$. Under a condition on the time step $\Delta t$ of the same type as (4.20), there exists a constant $C>0$ such that the following error estimate holds:

$$
\begin{aligned}
& \max _{n=0, \ldots, N}\left(\left\|\vec{V}\left(t_{n}\right)-\vec{V}_{h}^{n}\right\|_{\mathrm{C}^{0}\left([0, T], \mathrm{L}^{2}(\Omega)^{3}\right)}^{2}+\left\|\overrightarrow{\tilde{\sigma}}\left(t_{n+\frac{1}{2}}\right)-\overrightarrow{\tilde{\sigma}}_{h}^{n+\frac{1}{2}}\right\|_{\mathrm{C}^{0}\left([0, T], \mathrm{L}^{2}(\Omega)^{6}\right)}^{2}\right)^{1 / 2} \\
\leq & C \Delta t^{\min (\alpha, 2)}\|(\vec{V}, \overrightarrow{\tilde{\sigma}})\|_{\mathrm{C}^{\max (\alpha, 3)}\left([0, T], \mathrm{L}^{2}(\Omega)^{9}\right)}+C h^{\min (s, k)}\|(\vec{V}, \overrightarrow{\tilde{\sigma}})\|_{\mathrm{C}^{0}\left([0, T], P \mathrm{H}^{s+1}(\Omega)^{9}\right)} .
\end{aligned}
$$

Proof. Let be $\vec{v}_{h}^{n}$ and $\vec{s}_{h}^{n+\frac{1}{2}}$ similarly defined as in (3.2) and (3.3). Then, for each tetrahedron $\mathcal{T}_{i}$, we set $\widehat{v}_{i}^{n}=\left(\widehat{v}_{i j}^{n}\right)_{1 \leq j \leq \mathrm{dof}}$ and $\widehat{s}_{i}^{n}=\left(\widehat{s}_{i j}^{n+\frac{1}{2}}\right)_{1 \leq j \leq \mathrm{dof}}$, and we note the column vectors $\widehat{v}^{n}=\left(\widehat{v}_{i}^{n}\right)_{1 \leq i \leq N_{T}}$ and $\widehat{s}^{n}=$ $\left(\widehat{s}_{i}^{n+\frac{1}{2}}\right)_{1 \leq i \leq \text { dof }}$. We are interested in the estimation of the following local consistency error:

$$
\epsilon_{h}^{n}=\left(\left\|\vec{v}_{h}^{n}-\vec{V}_{h}\left(t_{n}\right)\right\|_{\mathrm{L}^{2}(\Omega)^{3}}^{2}+\left\|\vec{s}_{h}^{n+\frac{1}{2}}-\overrightarrow{\tilde{\sigma}}_{h}\left(t_{n+\frac{1}{2}}\right)\right\|_{\mathrm{L}^{2}(\Omega)^{6}}^{2}\right)^{1 / 2}
$$


where $\left(\vec{V}_{h}, \overrightarrow{\tilde{\sigma}}_{h}\right) \in \mathrm{C}^{N+1}\left([0, T], X_{h}^{1} \times X_{h}^{2}\right)$ is the semi-discrete solution of (3.16), (3.17) and (5.6) and $\left(\widehat{v}^{n+1}, \widehat{s}^{n+\frac{3}{2}}\right)$ has been computed as follows for all $N$ (even):

$$
\left\{\begin{aligned}
M_{\rho} \frac{\widehat{v}^{n+1}-\widehat{\mathbb{V}}\left(t_{n}\right)}{\Delta t} & =Q_{N} \widehat{\sigma}\left(t_{n+\frac{1}{2}}\right)-R_{N} \widehat{\mathbb{V}}\left(t_{n+\frac{1}{2}}\right) \\
M_{\Lambda} \frac{\widehat{s}^{n+\frac{3}{2}}-\widehat{\sigma}\left(t_{n+\frac{1}{2}}\right)}{\Delta t} & =X_{N} \widehat{\mathbb{V}}\left(t_{n+1}\right)-Y_{N} \widehat{\sigma}\left(t_{n+1}\right)
\end{aligned}\right.
$$

Using Taylor formulae, there exist $\left.c_{n+\frac{1}{2}} \in\right] t_{n+\frac{1}{2}}, t_{n+1}\left[\right.$ and $\left.d_{n+\frac{1}{2}} \in\right] t_{n}, t_{n+\frac{1}{2}}[$ such that

$$
\begin{aligned}
\widehat{\mathbb{V}}\left(t_{n+1}\right) & =\sum_{k=0}^{N} \frac{1}{k !}\left(\frac{\Delta t}{2}\right)^{k} \partial_{t}^{k} \widehat{\mathbb{V}}\left(t_{n+\frac{1}{2}}\right)+\frac{1}{(N+1) !}\left(\frac{\Delta t}{2}\right)^{N+1} \partial_{t}^{N+1} \widehat{\mathbb{V}}\left(c_{n+\frac{1}{2}}\right), \\
\widehat{\mathbb{V}}\left(t_{n}\right) & =\sum_{k=0}^{N} \frac{(-1)^{k}}{k !}\left(\frac{\Delta t}{2}\right)^{k} \partial_{t}^{k} \widehat{\mathbb{V}}\left(t_{n+\frac{1}{2}}\right)-\frac{1}{(N+1) !}\left(\frac{\Delta t}{2}\right)^{N+1} \partial_{t}^{N+1} \widehat{\mathbb{V}}\left(d_{n+\frac{1}{2}}\right) .
\end{aligned}
$$

On the other hand, applying (5.42)-(5.44) in the following equality

$$
\begin{aligned}
M_{\rho}\left(\widehat{\mathbb{V}}\left(t_{n+1}\right)-\widehat{v}^{n+1}\right)= & M_{\rho}\left(\widehat{\mathbb{V}}\left(t_{n+1}\right)-\widehat{\mathbb{V}}\left(t_{n}\right)\right)+M_{\rho}\left(\widehat{\mathbb{V}}\left(t_{n}\right)-\widehat{v}^{n+1}\right) \\
= & 2 M_{\rho} \sum_{k=1, \text { odd }}^{N-1} \frac{1}{k !}\left(\frac{\Delta t}{2}\right)^{k} \partial_{t}^{k} \widehat{\mathbb{V}}\left(t_{n+\frac{1}{2}}\right)-\Delta t Q_{N} \widehat{\sigma}\left(t_{n+\frac{1}{2}}\right)+\Delta t R_{N} \widehat{\mathbb{V}}\left(t_{n+\frac{1}{2}}\right) \\
& +\frac{1}{(N+1) !}\left(\frac{\Delta t}{2}\right)^{N+1} M_{\rho}\left(\partial_{t}^{N+1} \widehat{\mathbb{V}}\left(c_{n+\frac{1}{2}}\right)+\partial_{t}^{N+1} \widehat{\mathbb{V}}\left(d_{n+\frac{1}{2}}\right)\right)
\end{aligned}
$$

Using the identities (3.32) and (3.34), we deduce that the three first terms vanish. Then for each tetrahedron $\mathcal{T}_{i}$, it holds

$$
\vec{V}_{i}\left(t_{n+1}\right)-\widehat{v}_{i}^{n+1}=\sum_{j=1}^{\text {dof }}\left(\widehat{\mathbb{V}}_{i j}\left(t_{n+1}\right)-\widehat{v}_{i j}^{n+1}\right) \vec{\varphi}_{i j}=\frac{1}{(N+1) !}\left(\frac{\Delta t}{2}\right)^{N+1}\left(\partial_{t}^{N+1} \vec{V}_{i}\left(c_{n+\frac{1}{2}}\right)+\partial_{t}^{N+1} \vec{V}_{i}\left(d_{n+\frac{1}{2}}\right)\right) .
$$

Therefore, there exists a constant $C>0$ such that:

$$
\left\|\vec{V}_{h}\left(t_{n+1}\right)-\widehat{v_{h}^{n+1}}\right\|_{\mathrm{L}^{2}(\Omega)^{3}} \leq C \Delta t^{N+1}\left\|\vec{V}_{h}\right\|_{\mathrm{C}^{N+1}\left([0, T], \mathrm{L}^{2}(\Omega)^{3}\right)} .
$$

In the very same way, we obtain analogous estimates for $\left\|\overrightarrow{\tilde{\sigma}}_{h}\left(t_{n+\frac{3}{2}}\right)-\widehat{s_{h}^{n+\frac{3}{2}}}\right\|_{\mathrm{L}^{2}(\Omega)^{6}}$. Finally, as $\left(\vec{V}_{h}, \overrightarrow{\tilde{\sigma}}_{h}\right)$ is a discrete approximation of $(\vec{V}, \overrightarrow{\tilde{\sigma}})$ by Theorem 5.8 , the estimation of the local consistency error is bounded in the following way:

$$
\varepsilon_{h}^{n} \leq C \Delta t^{N+1}\|(\vec{V}, \overrightarrow{\tilde{\sigma}})\|_{\mathrm{C}^{N+1}\left([0, T], \mathrm{L}^{2}(\Omega)^{9}\right)} .
$$

On the other hand, according to (5.42), we also have

$$
\left\{\begin{aligned}
M_{\rho} \frac{\widehat{\mathbb{V}}\left(t_{n+1}\right)-\widehat{\mathbb{V}}\left(t_{n}\right)}{\Delta t} & =M_{\rho} \frac{\widehat{\mathbb{V}}\left(t_{n+1}\right)-\widehat{v}^{n+1}}{\Delta t}+Q_{N} \widehat{\sigma}\left(t_{n+\frac{1}{2}}\right)-R_{N} \widehat{\mathbb{V}}\left(t_{n+\frac{1}{2}}\right), \\
M_{\Lambda} \frac{\widehat{\sigma}\left(t_{n+\frac{3}{2}}\right)-\widehat{\sigma}\left(t_{n+\frac{1}{2}}\right)}{\Delta t} & =M_{\Lambda} \frac{\widehat{\sigma}\left(t_{n+\frac{3}{2}}\right)-\widehat{s}^{n+\frac{3}{2}}}{\Delta t}+X_{N} \widehat{\mathbb{V}}\left(t_{n+1}\right)-Y_{N} \widehat{\sigma}\left(t_{n+1}\right) .
\end{aligned}\right.
$$


Then, using (3.33) and (5.49), the field $\left(\widehat{\mathscr{V}}^{n}, \widehat{\mathscr{S}}^{n}+\frac{1}{2}\right)=\left(\widehat{\mathbb{V}}\left(t_{n}\right)-\widehat{\mathbb{V}}^{n}, \widehat{\sigma}\left(t_{n+\frac{1}{2}}\right)-\widehat{\sigma}^{n+\frac{1}{2}}\right)$ satisfies the following set of equations:

$$
\left\{\begin{array}{c}
M_{\rho} \frac{\widehat{\mathscr{V}}^{n+1}-\widehat{\mathscr{V}}^{n}}{\Delta t}=M_{\rho} \frac{\widehat{\mathbb{V}}\left(t_{n+1}\right)-\widehat{v}^{n+1}}{\Delta t}+Q_{N} \widehat{\mathscr{S}}^{n+\frac{1}{2}}-R_{N}\left[\widehat{\mathbb{V}}\left(t_{n+\frac{1}{2}}\right)-\widehat{\mathbb{V}}^{\left[n+\frac{1}{2}\right]}\right], \\
M_{\Lambda} \frac{\widehat{\mathscr{S}^{n}+\frac{3}{2}}-\widehat{\mathscr{S}^{n}+\frac{1}{2}}}{\Delta t}=M_{\Lambda} \frac{\widehat{\sigma}\left(t_{n+\frac{3}{2}}\right)-\widehat{s}^{n+\frac{3}{2}}}{\Delta t}+X_{N} \widehat{\mathscr{V}}^{n+1}-Y_{N}\left[\widehat{\sigma}\left(t_{n+1}\right)-\widehat{\sigma}^{[n+1]}\right] .
\end{array}\right.
$$

(1) Firstly, we consider the case where $N$ is even and the domain $\Omega$ with free surface boundary conditions only. Then, $R_{N}=0$ and $Y_{N}=0$ in (5.50) by Remark 3.4, and we introduce the error energy $\mathscr{E}^{n}$ at time $n \Delta t$ by:

$$
\mathscr{E}^{n}=\frac{1}{2}\left[\left(\widehat{\mathscr{V}}^{n}\right)^{t} M_{\rho} \widehat{\mathscr{V}}^{n}+\left(\widehat{\mathscr{S}}^{n}+\frac{1}{2}\right)^{t} M_{\Lambda} \widehat{\mathscr{S}}^{n}-\frac{1}{2}\right] .
$$

Reasoning similarly as for the discrete energy $E^{n}$ at (4.1), we can prove the following estimate under a condition of the same type as (4.19) and thanks to (5.48): there exists $C>0$ such that

$$
\left(\left\|\mathscr{V}_{h}^{n}\right\|_{\mathrm{L}^{2}(\Omega)^{3}}^{2}+\left\|\mathscr{S}_{h}^{n+\frac{1}{2}}\right\|_{\mathrm{L}^{2}(\Omega)^{6}}^{2}\right)^{1 / 2} \leq C \Delta t^{N}\|(\vec{V}, \overrightarrow{\tilde{\sigma}})\|_{\mathrm{C}^{N+1}\left([0, T], \mathrm{L}^{2}(\Omega)^{9}\right)} .
$$

Finally, we can conclude that the order of convergence of the scheme is $\mathcal{O}\left(\Delta t^{N}+h^{\min (s, k)}\right)$ by using the triangular inequality and results already established in the semi-discrete case (see Thm. 5.8).

(2) Then, for domains with absorbing boundary conditions (or mixed boundary conditions), we set $N=2$. Therefore, it remains to evaluate the following term in (5.50) with (3.28) and $\alpha \in\{1,2,3,4\}$ :

$$
\widehat{\mathbb{V}}\left(t_{n+\frac{1}{2}}\right)-\widehat{\mathbb{V}}\left[n+\frac{1}{2}\right]=\left(\widehat{\mathbb{V}}\left(t_{n+\frac{1}{2}}\right)-\sum_{i=1}^{\alpha} b_{i} \widehat{\mathbb{V}}\left(t_{n+1-i}\right)\right)+\widehat{\mathscr{V}}\left[n+\frac{1}{2}\right] .
$$

For $\left(\vec{V}_{h}, \overrightarrow{\tilde{\sigma}}_{h}\right) \in \mathrm{C}^{\max (\alpha, 3)}\left([0, T], X_{h}^{1} \times X_{h}^{2}\right)$, we know by a Taylor formula that there exists $\xi_{i, n+\frac{1}{2}} \in$ ]$t_{n+1-i}, t_{n+\frac{1}{2}}[$, with $i \in[1, \ldots, \alpha]$, such that

$$
\widehat{\mathbb{V}}\left(t_{n+1-i}\right)=\sum_{k=0}^{\alpha-1} \frac{1}{k !}\left(\frac{1}{2}-i\right)^{k} \partial_{t}^{k} \widehat{\mathbb{V}}\left(t_{n+\frac{1}{2}}\right)+\frac{1}{\alpha !}\left(\frac{1}{2}-i\right)^{\alpha} \Delta t^{\alpha} \partial_{t}^{\alpha} \widehat{\mathbb{V}}\left(\xi_{i, n+\frac{1}{2}}\right),
$$

which implies according to (3.29) that:

$$
\sum_{i=1}^{\alpha} b_{i} \widehat{\mathbb{V}}\left(t_{n+1-i}\right)=\widehat{\mathbb{V}}\left(t_{n+\frac{1}{2}}\right)+\frac{\Delta t^{\alpha}}{\alpha !}\left[\sum_{i=1}^{\alpha} b_{i}\left(\frac{1}{2}-i\right)^{\alpha} \partial_{t}^{\alpha} \widehat{\mathbb{V}}\left(\xi_{i, n+\frac{1}{2}}\right)\right] .
$$

And, finally, in a similar way for the term $\widehat{\sigma}\left(t_{n+1}\right)-\widehat{\sigma}^{[n+1]}$, the system (5.50) can be rewritten as

$$
\begin{aligned}
M_{\rho} \frac{\widehat{\mathscr{V}}^{n+1}-\widehat{\mathscr{V}}^{n}}{\Delta t}= & Q \widehat{\mathscr{S}}^{n+\frac{1}{2}}-R \widehat{\mathscr{V}}^{\left[n+\frac{1}{2}\right]}+M_{\rho} \frac{\widehat{\mathbb{V}}\left(t_{n+1}\right)-\widehat{v}^{n+1}}{\Delta t} \\
& +\frac{\Delta t^{\alpha}}{\alpha !} R\left[\sum_{i=1}^{\alpha} b_{i}\left(\frac{1}{2}-i\right)^{\alpha} \partial_{t}^{\alpha} \widehat{\mathbb{V}}\left(\xi_{i, n+\frac{1}{2}}\right)\right], \\
M_{\Lambda} \frac{\widehat{\mathscr{S}}^{n+\frac{3}{2}}-\widehat{\mathscr{S}^{n}+\frac{1}{2}}}{\Delta t}= & X \widehat{\mathscr{V}}^{n+1}-Y \widehat{\mathscr{S}}^{n+\frac{1}{2}}+M_{\Lambda} \frac{\widehat{\sigma}\left(t_{n+\frac{3}{2}}\right)-\widehat{s}^{n+\frac{3}{2}}}{\Delta t} \\
& +\frac{\Delta t^{\alpha}}{\alpha !} Y\left[\sum_{i=1}^{\alpha} b_{i}\left(\frac{1}{2}-i\right)^{\alpha} \partial_{t}^{\alpha} \widehat{\sigma}\left(\xi_{i, n+1}\right)\right] .
\end{aligned}
$$


Now, when $\alpha=1$, we introduce the error energy $\mathscr{E}^{n}$ at time $n \Delta t$ by:

$$
\mathscr{E}^{n}=\frac{1}{2}\left[\left(\widehat{\mathscr{V}}^{n}\right)^{t} M_{\rho} \widehat{\mathscr{V}}^{n}+\left(\widehat{\mathscr{S}}^{n}+\frac{1}{2}\right)^{t} M_{\Lambda} \widehat{\mathscr{S}}^{n-\frac{1}{2}}\right]-\frac{\Delta t}{4}\left[\left(\widehat{\mathscr{V}}^{n}\right)^{t} R \widehat{\mathscr{V}}^{n}-\left(\widehat{\mathscr{S}}^{n-\frac{1}{2}}\right)^{t} Y \widehat{\mathscr{S}}^{n-\frac{1}{2}}\right],
$$

elsewhere, for $\alpha \in\{2,3,4\}, \mathscr{E}^{n}$ is defined by:

$$
\begin{aligned}
\mathscr{E}^{n}= & \frac{1}{2}\left[\left(\widehat{\mathscr{V}}^{n}\right)^{t} M_{\rho} \widehat{\mathscr{V}}^{n}+\left(\widehat{\mathscr{S}}^{n}+\frac{1}{2}\right)^{t} M_{\Lambda} \widehat{\mathscr{S}}^{n-\frac{1}{2}}\right] \\
& -\frac{\Delta t}{4} \sum_{i=1}^{\alpha}\left|b_{i}\right|\left[\left(\widehat{\mathscr{V}}^{n}\right)^{t} R \widehat{\mathscr{V}}^{n}-\left(\widehat{\mathscr{S}}^{n}+\frac{1}{2}-i\right)^{t} Y \widehat{\mathscr{S}}^{n}+\frac{1}{2}-i\right] \\
& +\frac{\Delta t}{4} \sum_{i=2}^{\alpha}\left|b_{i}\right|\left(\widehat{\mathscr{V}}^{n}-\gamma_{i} \widehat{\mathscr{V}}^{n+1-i}\right)^{t} R\left(\widehat{\mathscr{V}}^{n}-\gamma_{i} \widehat{\mathscr{V}}^{n+1-i}\right) .
\end{aligned}
$$

Reasoning similarly as for the discrete corrected energy $\mathcal{E}^{n}$ at (4.7) or (4.8), we can prove the following estimate under a condition of the same type as (4.20) and thanks to (5.48): there exists $C>0$ such that

$$
\left(\left\|\mathscr{V}_{h}^{n}\right\|_{\mathrm{L}^{2}(\Omega)^{3}}^{2}+\left\|\mathscr{S}_{h}^{n+\frac{1}{2}}\right\|_{\mathrm{L}^{2}(\Omega)^{6}}^{2}\right)^{1 / 2} \leq C\left(\Delta t^{\alpha}\|(\vec{V}, \overrightarrow{\tilde{\sigma}})\|_{\mathrm{C}^{\alpha}\left([0, T], \mathrm{L}^{2}(\Omega)^{9}\right)}+\Delta t^{2}\|(\vec{V}, \overrightarrow{\tilde{\sigma}})\|_{\mathrm{C}^{3}\left([0, T], \mathrm{L}^{2}(\Omega)^{9}\right)}\right) .
$$

Finally, we can conclude that the order of convergence of the scheme is $\mathcal{O}\left(\Delta t^{\min (\alpha, 2)}+h^{\min (s, k)}\right)$ by using the triangular inequality and results already established in the semi-discrete case (see Thm. 5.8).

\section{Numerical Results}

The DG method has been applied to several numerical problems, each to illustrate a specific property. First, the propagation of an eigenmode aims to realise a convergence study of the schemes and a comparison with the theoretical analysis. Moreover, it allows validating the free surface conditions and comparing the properties of the standard and fourth-order leap-frog schemes. The second study concerns the propagation of a pulse and focuses on the absorbing boundary conditions. The last application concerns the propagation of the wave produced by an explosive source in a half space which enables, firstly, validating the source introduction and apply the method in a more realistic context.

\subsection{Convergence study. Propagation of an eigenmode in $3 \mathrm{D}$}

The first problem concerns the propagation of an eigenmode in three dimensions of space. The domain of computation is the unit cubic cavity on which we apply free surface boundary conditions. We are interested in the $(1,1,1)$ mode whose exact solution at time $t$ and in $X=(x, y, z)$ is given by:

$$
\left\{\begin{aligned}
V_{x}(t, X) & =\cos (\pi x)[\sin (\pi y)-\sin (\pi z)] \cos (\Omega t), \\
V_{y}(t, X) & =\cos (\pi y)[\sin (\pi z)-\sin (\pi x)] \cos (\Omega t), \\
V_{z}(t, X) & =\cos (\pi z)[\sin (\pi x)-\sin (\pi y)] \cos (\Omega t), \\
\sigma_{x x}(t, X) & =-A \sin (\pi x)[\sin (\pi y)-\sin (\pi z)] \sin (\Omega t), \\
\sigma_{y y}(t, X) & =-A \sin (\pi y)[\sin (\pi z)-\sin (\pi x)] \sin (\Omega t), \\
\sigma_{z z}(t, X) & =-A \sin (\pi z)[\sin (\pi x)-\sin (\pi y)] \sin (\Omega t), \\
\sigma_{x y}(t, X) & =\sigma_{x z}(t, X)=\sigma_{y z}(t, X)=0,
\end{aligned}\right.
$$

with $A=\sqrt{2 \rho \mu}$ and $\Omega=\pi \sqrt{2 \mu / \rho}$. We set dimensionless values for the medium properties, $\rho=1.0, \lambda=0.5$ and $\mu=0.25$, which implies that $v_{p}=1$. and $v_{s}=0.5$. The initialisation of the leap-frog scheme is realized 
TABLE 1. Characteristics of the uniform meshes used for the convergence study; $N_{N}$ and $N_{T}$ are respectively the total number of nodes and tetraedra of the meshes.

\begin{tabular}{cccccccc}
\hline & M1 & M2 & M3 & M4 & M5 & M6 & M7 \\
\hline$h_{\min }$ & 0.5 & 0.25 & 0.125 & $8.33 \times 10^{-2}$ & $6.25 \times 10^{-2}$ & $5.0 \times 10^{-2}$ & $3.12 \times 10^{-2}$ \\
$h=h_{\max }$ & 0.866 & 0.433 & 0.216 & 0.144 & 0.108 & $8.66 \times 10^{-2}$ & $5.41 \times 10^{-2}$ \\
$N_{N}$ & 27 & 125 & 729 & 2197 & 4913 & 9261 & 35937 \\
$N_{T}$ & 48 & 84 & 3072 & 10368 & 24576 & 48000 & 19660 \\
\hline
\end{tabular}

by deducing, from the analytical expressions (6.1), values for the velocity components at $t=0$ and for stress components at $t=\frac{\Delta t}{2}$. We introduce a $\mathrm{L}^{2}$-error, at step $n$, between the exact value and the solution of the numerical scheme in the unit cube which depends on velocity components $V_{\alpha}(\alpha=x, y, z)$ at $n \Delta t$ and stress components $\sigma_{\alpha \beta}(\alpha, \beta=x, y, z)$ at $\left(n+\frac{1}{2}\right) \Delta t$ and writes

$$
\operatorname{err}_{\mathrm{L}^{2}}^{n}=\sqrt{\sum_{i=1}^{N_{T}} \int_{\mathcal{T}_{i}}\left[\sum_{\alpha \in\{x, y, z\}}\left(V_{\alpha}\left(n \Delta t, x_{i}\right)-\left(V_{\alpha}\right)_{i}^{n}\right)^{2}+\sum_{\alpha, \beta \in\{x, y, z\}}\left(\sigma_{\alpha \beta}\left((n+1 / 2) \Delta t, x_{i}\right)-\left(\sigma_{\alpha \beta}\right)_{i}^{n+1 / 2}\right)^{2}\right] .}
$$

Series of different uniform meshes are constructed by dividing the domain in cubic cells which are split in six tetraedra. The mesh spacing $h$ is the length of the longest edge of the mesh. The characteristics of all meshes can be found in Table 1. Different methods have been used, the notation $\mathcal{P}_{p}$ refering to a discretization based on polynomials of degree $p$. Two leap-frog schemes are also compared: the standard one, second-order accurate (refered as LF2), and a fourth-order extension (named LF4 in the following). Simulations are performed until $t_{\max }=142 \mathrm{~s}$ which corresponds to 50 periods in the cube and is a sufficiently long time to distinguish the results from different time and space discretizations.

For all the applications, the time-step of the simulation is the minimum of all local time-steps calculated in each tetraedron using the following formula

$$
\Delta t_{L F 2}=\min _{\mathcal{T}_{i}} \Delta t_{i} \quad \text { with } \quad \Delta t_{i}=\frac{1}{2 p+1} \frac{\bar{h}_{i}}{\left.v_{p}\right|_{i}} \quad \text { and } \quad \Delta t_{L F 4}=2.5 \times \Delta t_{L F 2},
$$

which depends on the space approximation $p, \bar{h}$, the smallest height in the tetraedron and the local value of $v_{p}$. The ratio between $\Delta t_{L F 4}$ and $\Delta t_{L F 2}$ has been deduced experimentally in [35].

We present in Figure 1 the results of the convergence study. First, Figures 1a and 1b display the maximum value of the numerical error $\operatorname{err}_{\mathrm{L}^{2}}$, at time $t_{\max }$, and in logarithmic scale by respect to the mesh spacing $h$ for LF2 and LF4 leap-frog schemes respectively. The corresponding results of efficiency, i.e. the error as a function of the CPU time, are presented in Figures $1 \mathrm{c}$ and $1 \mathrm{~d}$. The values of the error, the corresponding convergence orders and the CPU times are specified in Table 2 and complete these figures. As can be noticed, some simulations are missing, those corresponding to the highest-degree space approximations and the finest meshes, simply for reason of CPU cost (these calculations have been done on a standard PC). In contrast, the solutions of the $\mathcal{P}_{1}$ method show significant dispersion errors for the coarsest meshes in long time (the solution can be shifted more than over a period) and have been excluded for this reason.

When examining Figures 1a and 1b, we notice that the results are in accordance with the theoretical analysis. For a method $\mathcal{P}_{p^{-}} \mathrm{LF}_{i}$, in presence of free-surface conditions only, the error is $\mathcal{O}\left(h^{p}+\Delta t^{i}\right)$. Then, for $p \leq 2$, the global convergence rate is about $p$ and dominated by the one in space, regardless of the leap-frog scheme; this is what is observed in both figures and confirmed by the values in Table 2. For the standard leap-frog scheme (LF2) and higher values of $p$, the convergence rate is still equal to 2 because dominated by the one in time. The improvement brought by the LF4 leap-frog extension is clear since, for $p \geq 3$, the convergence rate is no 
(a)

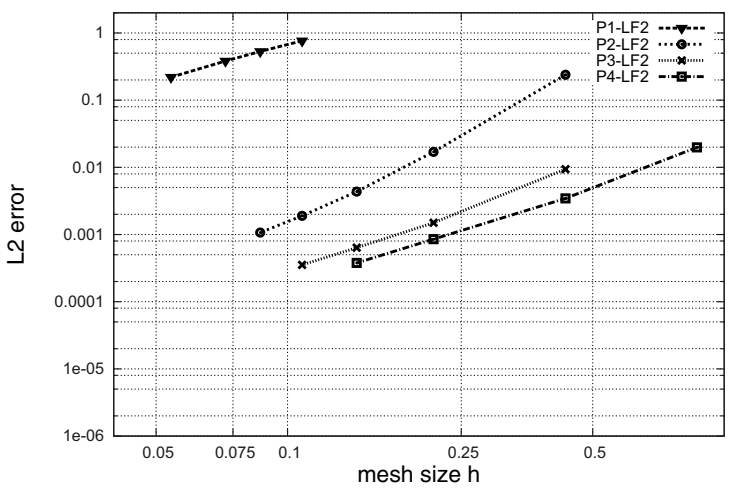

(c)

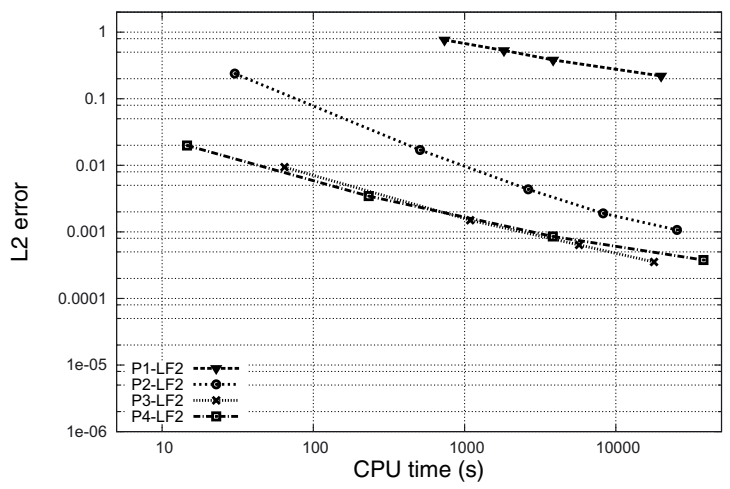

(b)

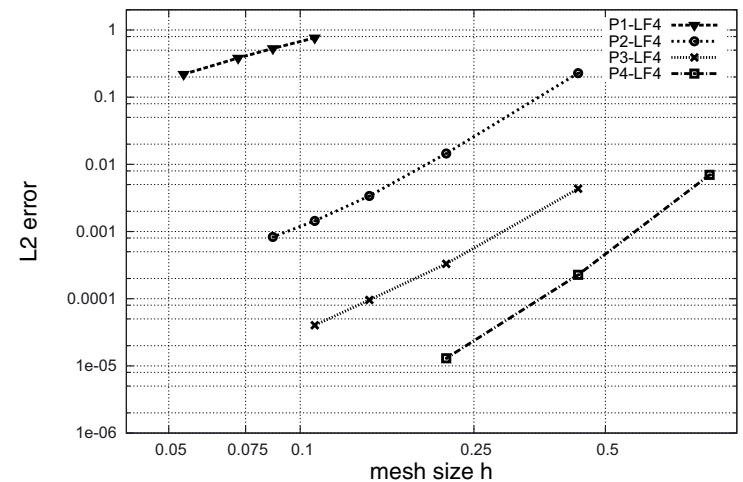

(d)

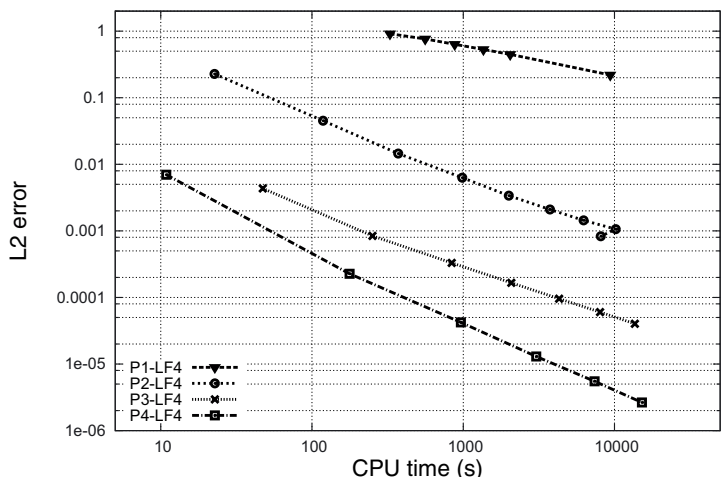

Figure 1. Convergence study for the propagation of an eigenmode. Error as a function of the mesh size $h$ ( $\mathrm{a}$ and $\mathrm{b}$ ) and CPU time (c and d) for different $\mathcal{P}_{p}-\mathrm{LF}_{i}$ methods $(p=1, \ldots, 4, i=$ $2,4)$ and leap-frog time schemes (left column for LF2 and right column for LF4, respectively). Errors at $t_{\max }=142 \mathrm{~s}$ using uniform meshes.

more constrained by the time scheme and is also equal to $p$, as noticeable in Figure $1 \mathrm{~b}$ as well as in convergence orders of Table 2. Note that this study also validates the implementation of the free surface condition.

We are now interested in the efficiency of the different methods presented in Figures 1c and 1d. We remark that, to reach a given error level, methods based on high order polynomials generally need lower CPU times, for both types of leap-frog schemes. However, when using the standard leap-frog scheme, the $\mathcal{P}_{4}$ method becomes too expensive compared to the $\mathcal{P}_{3}$ scheme, especially for fine meshes, mainly because of the limitation in convergence due to the time scheme and to a larger number of degrees of freedom. The improvement brought by the high-order leap-frog (LF4) extension is obvious and can be explained by better convergence rates for $p \geq 3$ and, also, by the use of greater time-steps. So, even if it is based on a multi-step algorithm, the additional iterations are counterbalanced by a greater time-step which makes the LF4 method more efficient. This is confirmed by the CPU times given in Table 2 proving a reduction of about $25 \%$ of the CPU time between results corresponding to LF2 and LF4 methods, even for $p=1,2$ for which error levels are comparable for LF2 and LF4 time discretizations.

In order to complete the comparison between both LF2 and LF4 leap-frog schemes, we plot in Figure 2 the error until $t_{\max }$, obtained using the mesh $\mathrm{M} 4$, for various $\mathcal{P}_{p}$ methods $(2 \leq p \leq 4)$. Figures $2 \mathrm{a}$ and $2 \mathrm{~b}$ corresponding to $p=2$ and 3 , justify the choice of a long time for simulations. For $p=2$, the difference due to the time approximation is very low and only visible after $30 \mathrm{~s}$. The difference between time methods is more 
TABLE 2. Errors in $L^{2}$-norm, CPU times and convergence orders corresponding to the convergence study, $\mathcal{P}_{p}$-LF $i$ methods $(p=1, \ldots, 4, i=2,4)$.

\begin{tabular}{|c|c|c|c|c|c|c|c|c|c|c|c|c|}
\hline & \multicolumn{3}{|c|}{$\mathcal{P}_{1-\mathrm{LF} 2}$} & \multicolumn{3}{|c|}{$\mathcal{P}_{2}$-LF2 } & \multicolumn{3}{|c|}{$\mathcal{P}_{3}-\mathrm{LF} 2$} & \multicolumn{3}{|c|}{$\mathcal{P}_{4}$-LF2 } \\
\hline & $e r r_{L^{2}}$ & $\mathcal{O}_{L^{2}}$ & $\mathrm{CPU}(\mathrm{s})$ & $e r r_{L^{2}}$ & $\mathcal{O}_{L^{2}}$ & $\mathrm{CPU}(\mathrm{s})$ & $e r r_{L^{2}}$ & $\mathcal{O}_{L^{2}}$ & $\mathrm{CPU}(\mathrm{s})$ & $e r r_{L^{2}}$ & $\mathcal{O}_{L^{2}}$ & $\mathrm{CPU}(\mathrm{s})$ \\
\hline M1 & & - & & & - & & & - & & $1.98 \times 10^{-2}$ & - & 14. \\
\hline M2 & & - & & $2.39 \times 10^{-1}$ & - & 30. & $9.40 \times 10^{-3}$ & - & 64. & $3.45 \times 10^{-3}$ & 2.52 & 232. \\
\hline M3 & & - & & $1.70 \times 10^{-2}$ & 3.80 & 507. & $1.50 \times 10^{-3}$ & 2.64 & 1090. & $8.53 \times 10^{-4}$ & 2.01 & 3829. \\
\hline M4 & & - & & $4.36 \times 10^{-3}$ & 3.35 & 2637. & $6.38 \times 10^{-4}$ & 2.10 & 5716. & $3.78 \times 10^{-4}$ & 2.00 & 38300 . \\
\hline M5 & $7.65 \times 10^{-1}$ & - & 736. & $1.90 \times 10^{-3}$ & 2.88 & 8251. & $3.54 \times 10^{-4}$ & 2.04 & 17894. & & - & \\
\hline M6 & $32 \times 10^{-1}$ & 1.62 & 1821. & $1.07 \times 10^{-3}$ & 2.57 & 25392. & & - & & & - & \\
\hline \multirow[t]{3}{*}{ M7 } & $20 \times 10^{-1}$ & 1.80 & 19923. & & - & & & - & & & - & \\
\hline & \multicolumn{3}{|c|}{$\mathcal{P}_{1}-\mathrm{LF} 4$} & \multicolumn{3}{|c|}{$\mathcal{P}_{2}$-LF4 } & \multicolumn{3}{|c|}{$\overline{\mathcal{P}_{3} \text {-LF4 }}$} & \multicolumn{3}{|c|}{$\mathcal{P}_{4}$-LF4 } \\
\hline & $e r r_{L^{2}}$ & $\overline{\mathcal{O}_{L^{2}}}$ & $\mathrm{CPU}(\mathrm{s})$ & $e r r_{L^{2}}$ & $\overline{\mathcal{O}_{L^{2}}}$ & CPU (s) & $e r r_{L^{2}}$ & $\mathcal{O}_{L^{2}}$ & $\mathrm{CPU}(\mathrm{s})$ & $e r r_{L^{2}}$ & $\mathcal{O}_{L^{2}}$ & $\mathrm{CPU}(\mathrm{s})$ \\
\hline M1 & & - & & & - & & & - & & $6.98 \times 10^{-3}$ & - & 10. \\
\hline M2 & & - & & $2.28 \times 10^{-1}$ & - & 22 . & $4.35 \times 10^{-3}$ & - & 47. & $2.27 \times 10^{-4}$ & 4.94 & 177. \\
\hline M3 & & - & & $1.45 \times 10^{-2}$ & 3.97 & 371. & $3.30 \times 10^{-4}$ & 3.72 & 838. & $1.30 \times 10^{-5}$ & 4.12 & 3038. \\
\hline M4 & & - & & $3.38 \times 10^{-3}$ & 3.59 & 2002. & $9.59 \times 10^{-5}$ & 3.04 & 4303. & $2.65 \times 10^{-6}$ & 3.92 & 15192. \\
\hline M5 & $7.64 \times 10^{-1}$ & - & 560. & $1.44 \times 10^{-3}$ & 2.96 & 6255. & $4.03 \times 10^{-5}$ & 3.01 & 13637. & & - & \\
\hline M6 & $5.32 \times 10^{-1}$ & 1.62 & 1360. & $8.29 \times 10^{-4}$ & 2.47 & 8122. & & - & & & - & \\
\hline M7 & $2.20 \times 10^{-1}$ & 1.80 & 9375. & & - & & & - & & & - & \\
\hline
\end{tabular}

visible for the $\mathcal{P}_{3}$ method, in Figure 2b; for times greater than $10 \mathrm{~s}$, the error associated to the standard leap-frog scheme increases continuously since it remains quite constant with the LF4 extension. The improvement due to the LF4 leap-frog scheme is clear in Figures 2c and 2d, corresponding to the $\mathcal{P}_{4}$ method. The high-order leap-frog extension allows a reduction of the error by a factor of about 100 and proves the interest of using high-order space approximations.

Finally, to conclude this study, we plot in Figure 3, the value of the velocity $V_{x}$ recorded inside the domain, at location $(0.25,0.5,0.25)$, over a long time, corresponding to 200 and 400 periods approximately. We compare the exact solution (series of dots) to the results obtained using the LF2 and LF4 leap-frog schemes, with the coarse mesh M2 and the $\mathcal{P}_{4}$ method. For the first interval, Figure 3a, the results are very close. We can notice a slight delay of the LF2 solution compared to the two others. For the second interval, Figure 3b, which corresponds to a very long time, the difference between the solutions is clear. The dispersion error of the LF2 time scheme results in a much larger delay. Note that the amplitude is correct for both solutions and that the solution corresponding to the LF4 method is in perfect accordance with the exact one.

\subsection{Propagation of a pulse. Comparison between two types of absorbing boundary conditions}

The second problem we have studied is the propagation of a pulse in a homogeneous medium. Although this is a one-dimensional problem, it is possible to solve it in two or three dimensions of space. This is done here, in 3D, simply to apply the solver proposed in this paper. This study follows previous results presented by Delcourte et al. [25], using a two-dimensional method, that highlighted some limitations of the standard absorbing boundary conditions and aims to focus on the improvement proposed in Section 3.4.2. More precisely, we compare two absorbing boundary conditions among those defined by equations (3.30): one corresponding to $\alpha=1$ and refered as "standard absorbing condition" and a second one $(\alpha=2)$, based on two levels in time and called "modified absorbing condition" in the following. 
(a)

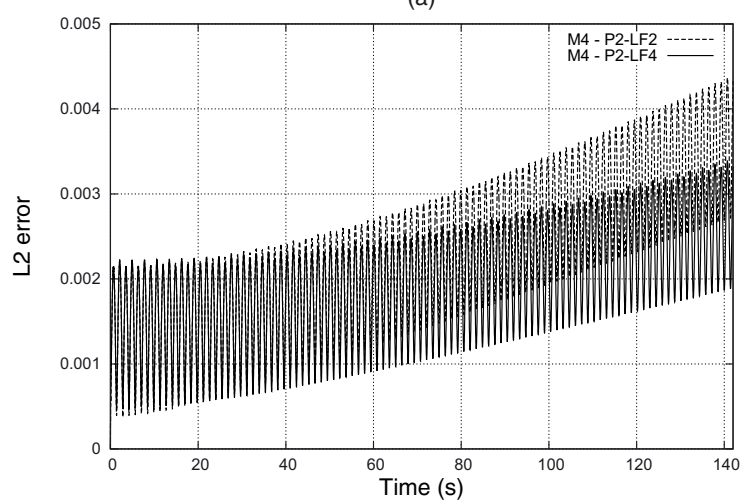

(c)

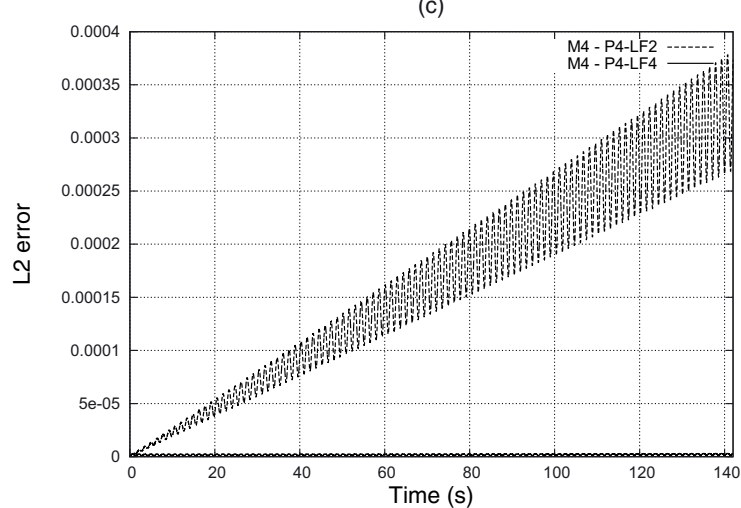

(b)

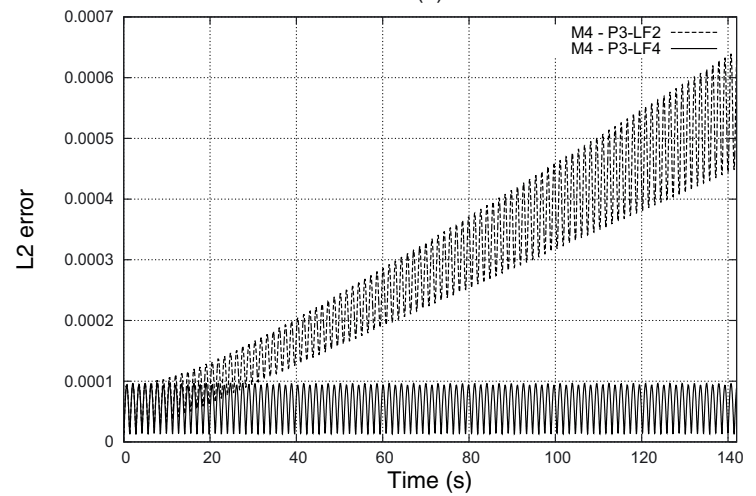

(d)

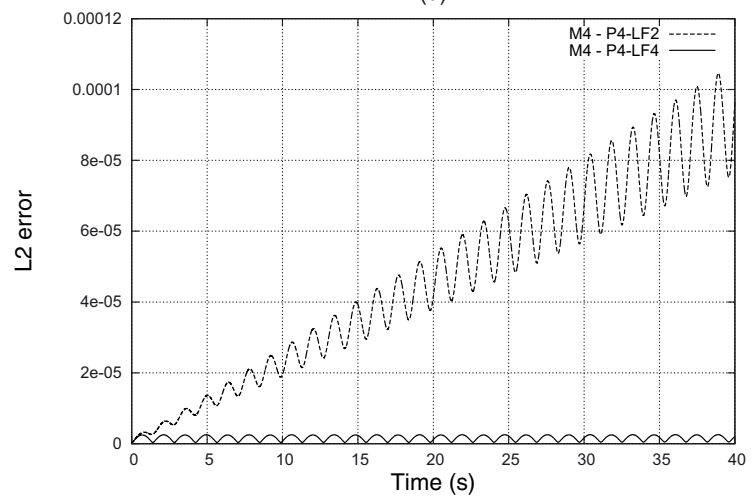

FiguRE 2. Errors in $L^{2}$-norm as a function of time for LF2 and LF4 leap-frog schemes. (a) $\mathcal{P}_{2}$ methods, (b) $\mathcal{P}_{3}$ methods, (c) $\mathcal{P}_{4}$ methods and (d) detail of (c) for $t \in[0 ; 40]$. All results correspond to mesh M4.

(a)

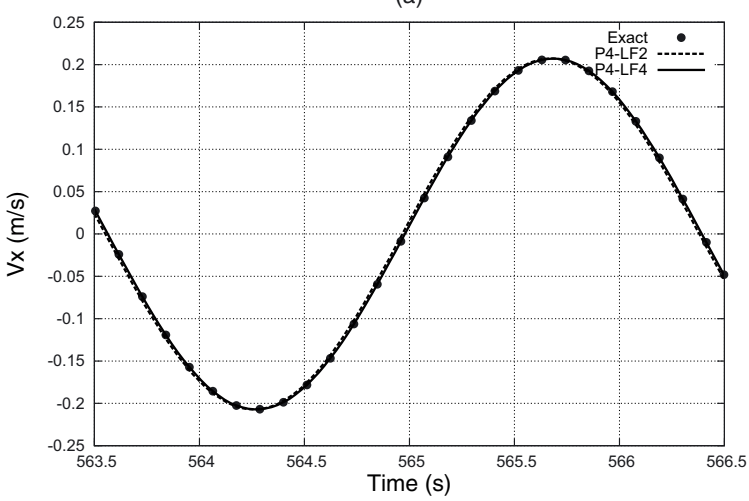

(b)

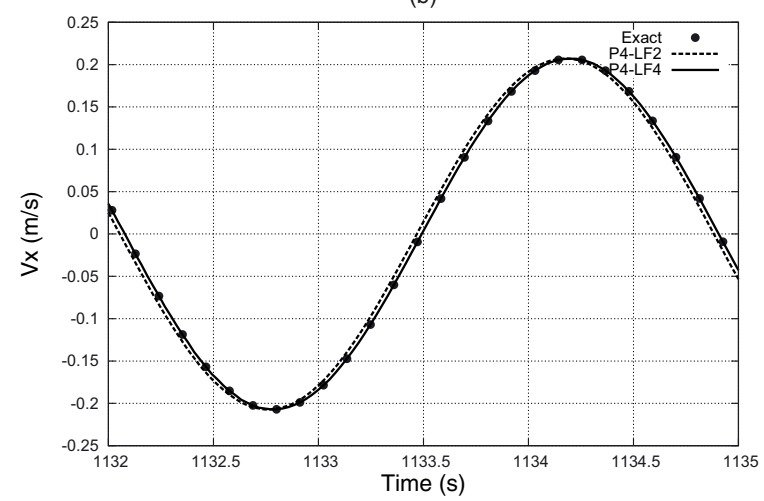

FiguRe 3. Velocity $V_{x}$ recorded at location $(0.25,0.5,0.25)$ as a function of time, for time intervals [563.5; 566.5] (a) and [1132.; 1135.] (b). $\mathcal{P}_{4}$ method, mesh M2 and comparison between LF2 (dashed lines) and LF4 (solid lines) leap-frog schemes. 


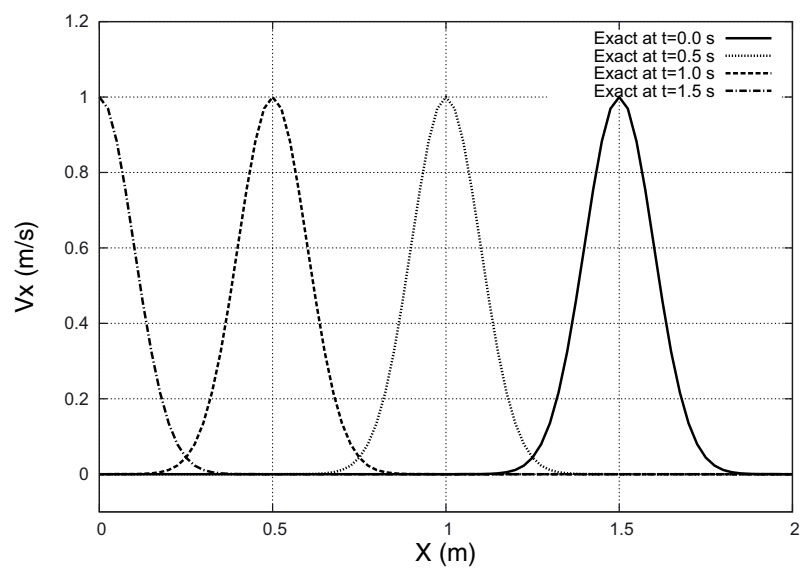

Figure 4. Exact solution for the pulse. Velocity $V_{x}$ at times $t=0.0,0.5,1.0$ and $1.5 \mathrm{~s}$.

The domain of computation is $[0 ; 2] \times[0 ; l]^{2}$; the length $l$ being variable in $y$ - and $z$-directions since uniform meshes are constructed always considering 5 nodes in these two directions, whatever the discretization chosen in the $x$-direction. As previously, dimensionless values are set for $\rho=1, \lambda=0.5$ and $\mu=0.25$ resulting in $v_{p}=1$. and $v_{s}=0.5$. The compression pulse is placed in $x_{0}=1.5$ and is defined by

$$
V_{x}\left(x, t_{0}=0\right)=\exp ^{-50\left(x-x_{0}\right)^{2}}, \quad \sigma_{x x}\left(x, t_{0}=0\right)=-\exp ^{-50\left(x-x_{0}\right)^{2}},
$$

and, for all $t$, the other components of velocity or stress are equal to 0 . The analytical solution is calculated using the characteristic method according to the initial condition at $t_{0}$ and expresses

$$
\left\{\begin{array}{l}
V_{x}(x, t)=\frac{1}{2}\left[V_{x}\left(x-v_{p} t, 0\right)-\frac{\sigma_{x x}\left(x-v_{p} t, 0\right)}{\rho v_{p}}\right]+\frac{1}{2}\left[V_{x}\left(x+v_{p} t, 0\right)+\frac{\sigma_{x x}\left(x+v_{p} t, 0\right)}{\rho v_{p}}\right], \\
\sigma_{x x}(x, t)=\frac{1}{2} \rho v_{p}\left[V_{x}\left(x+v_{p} t, 0\right)+\frac{\sigma_{x x}\left(x+v_{p} t, 0\right)}{\rho v_{p}}\right]-\frac{1}{2} \rho v_{p}\left[V_{x}\left(x-v_{p} t, 0\right)-\frac{\sigma_{x x}\left(x-v_{p} t, 0\right)}{\rho v_{p}}\right] .
\end{array}\right.
$$

The pulse propagates at $v_{p}$ and the exact solution for velocity $V_{x}$ at different times is shown in Figure 4.

We compare the solutions obtained using different $\mathcal{P}_{p}$ methods $(p=1, \ldots, 4)$ and both standard and modified absorbing boundary conditions. In this study, we use the standard leap-frog time scheme in order to focus on the absorbing boundary conditions. We present, in Figure 5, the $\mathrm{L}^{2}$-error as a function of time for the various methods. These results correspond to a uniform mesh containing 2025 nodes and 7680 tetraedra $\left(h_{\min }=\right.$ $2.5 \times 10^{-2}, h_{\max }=4.33 \times 10^{-2}$ and $\left.l=1.0 \times 10^{-1}\right)$. When examining the different figures, we first notice that all results have the same behavior that can be related to the evolution of the pulse during time, as shown in Figure 4 . The error increases rapidly and stabilizes around the value $t=1 \mathrm{~s}$ that corresponds to times for which the pulse propagates inside the computational domain. Note the vertical scale of each figure which indicates a global reduction of the error for higher values of $p$. For $t>1 \mathrm{~s}$, the error decreases as the pulse begins to go out of the domain. Until $t=1.5 \mathrm{~s}$, the error only depends on the accuracy of the method (i.e. the value of $p$ ) and not on the absorbing boundary condition. For times greater than $1.5 \mathrm{~s}$, the results corresponding to the two boundary conditions can be distinguished; the improvement brought by the modified absorbing condition is clearly visible. For $p \geq 2$, the error at $t=2 \mathrm{~s}$ is significantly lower with the modified absorbing condition which proves less spurious reflections than with the standard absorbing condition. It should also be noted that, when using the standard condition, the levels of error at $t=2 \mathrm{~s}$ corresponding to $2 \leq p \leq 4$ are very close which proves that this condition tends to reduce the convergence.

For a detailed comparison of the solutions of the two absorbing methods, we plot in Figure 6 the value of $V_{x}$ at $t=2 \mathrm{~s}$, as a function of $x$ in the middle of the domain, i.e. in $y=z=l / 2$. We compare the exact solution 
(a)

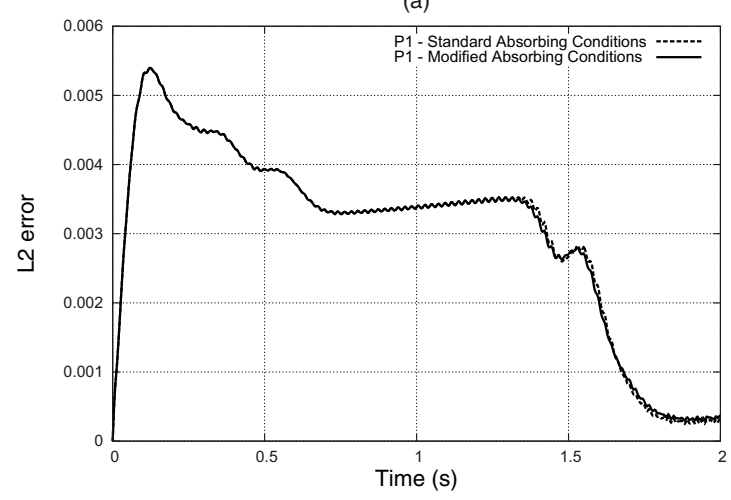

(c)

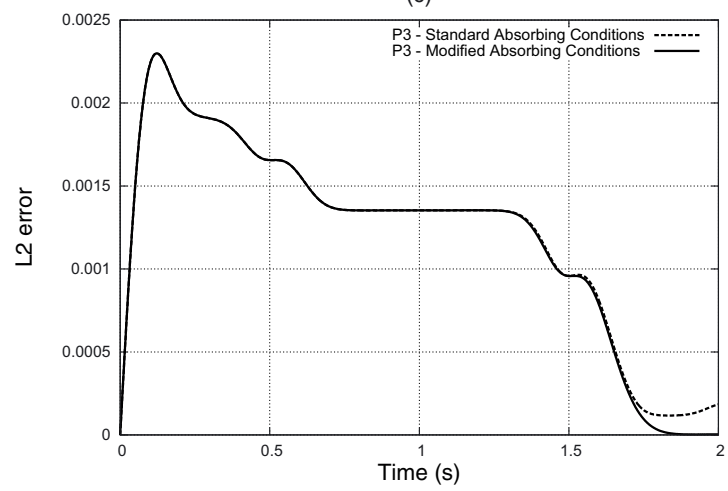

(b)

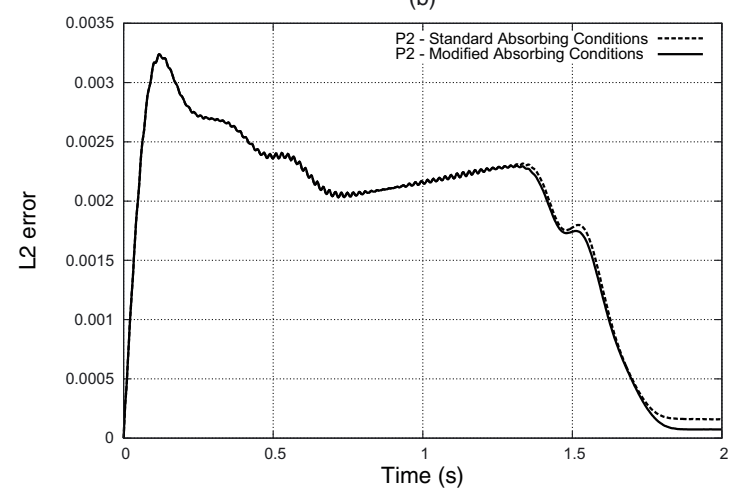

(d)

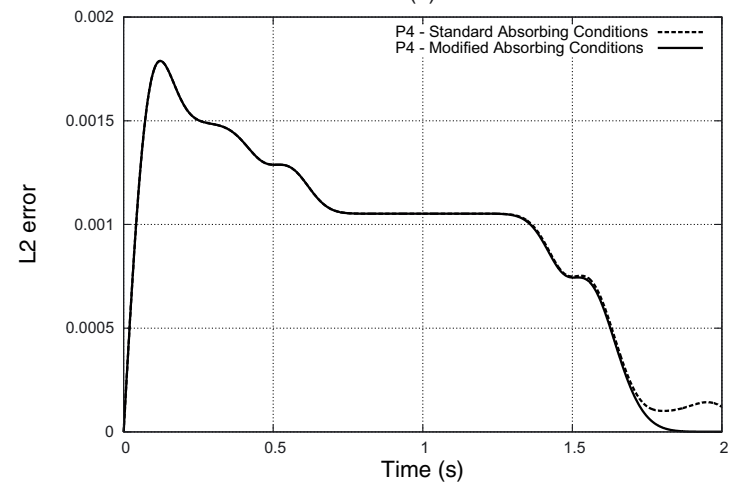

FiguRE 5. Errors in $L^{2}$-norm as a function of time for (a) $\mathcal{P}_{1}$, (b) $\mathcal{P}_{2}$, (c) $\mathcal{P}_{3}$ and (d) $\mathcal{P}_{4}$ methods. All results correspond to a uniform mesh of 2025 nodes and 7680 tetraedra. Dashed (respectively solid) lines for the standard (respectively modified) absorbing boundary condition.

(a series of dots) with the results of various $\mathcal{P}_{p}$ methods $(p=1, \ldots, 4)$ using the standard (dashed lines) and the modified (solid lines) absorbing conditions. At this time, the pulse is out of the computational domain and the various curves are a way to measure the level of accuracy of the boundary conditions. We present, in Figure 6a, the results of the $\mathcal{P}_{1}$ method: many spurious oscillations are visible and the two absorbing conditions lead to comparable error levels $\left(\max _{x}\left|V_{x}\right| \simeq 4.4 \times 10^{-4}\right)$. Better results are obtained using the $\mathcal{P}_{2}$ method, in Figure $6 \mathrm{~b}$ : the use of the modified absorbing condition reduces the amplitude of the oscillations $\left(\max _{x}\left|V_{x}\right| \simeq 2.5 \times 10^{-4}\right.$ with the standard and $\max _{x}\left|V_{x}\right| \simeq 9.1 \times 10^{-5}$ with the modified absorbing condition) but a distinct reflection is visible at about $x=0.15$ for both types of conditions. Figures $6 \mathrm{c}$ and $6 \mathrm{~d}$ compare the solutions for $\mathcal{P}_{3}$ and $\mathcal{P}_{4}$ methods. In both cases, reflections are visible with the standard absorbing condition even if the amplitude is slightly reduced for the $\mathcal{P}_{4}$ method $\left(\max _{x}\left|V_{x}\right| \simeq 2.0 \times 10^{-4}\right.$ for $\mathcal{P}_{3}$ method and $\max _{x}\left|V_{x}\right| \simeq 1.4 \times 10^{-4}$ for $\mathcal{P}_{4}$ method). The improvement brought by the modified absorbing condition is obvious and, at this scale, it is not possible to distinguish them from the exact solution. We plot in Figure 6e the results obtained using the $\mathcal{P}_{3}$ and $\mathcal{P}_{4}$ methods and the modified absorbing condition. Some spurious reflections remain but with amplitudes clearly lower $\left(\max _{x}\left|V_{x}\right| \simeq 6.8 \times 10^{-6}\right.$ for $\mathcal{P}_{3}$ method and $\max _{x}\left|V_{x}\right| \simeq 3.8 \times 10^{-6}$ for $\mathcal{P}_{4}$ method). This illustrates the improvement brought by for the absorbing boundary conditions proposed in Section 3.4.2. The comparison of the two formulations, corresponding to $\alpha=1$ and $\alpha=2$, shows a clear reduction of the spurious reflections at the boundary in a simple case where the wave propagates perpendiculary to the boundary. This condition has to be studied in a more complex configuration, in particular involving razing waves. However, note that the 
(a)

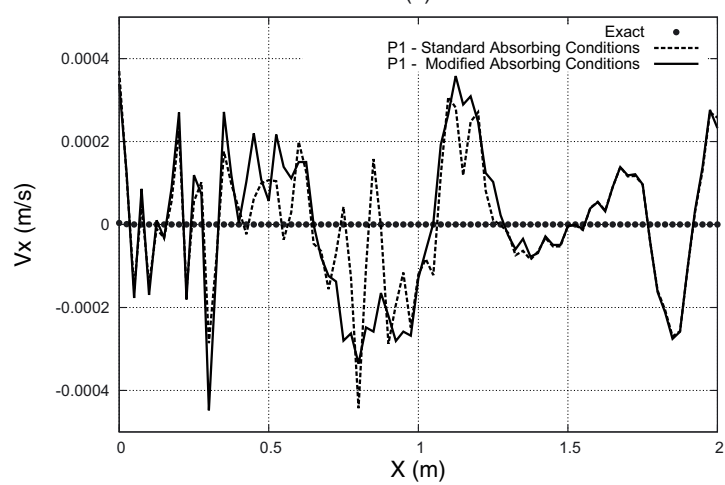

(c)
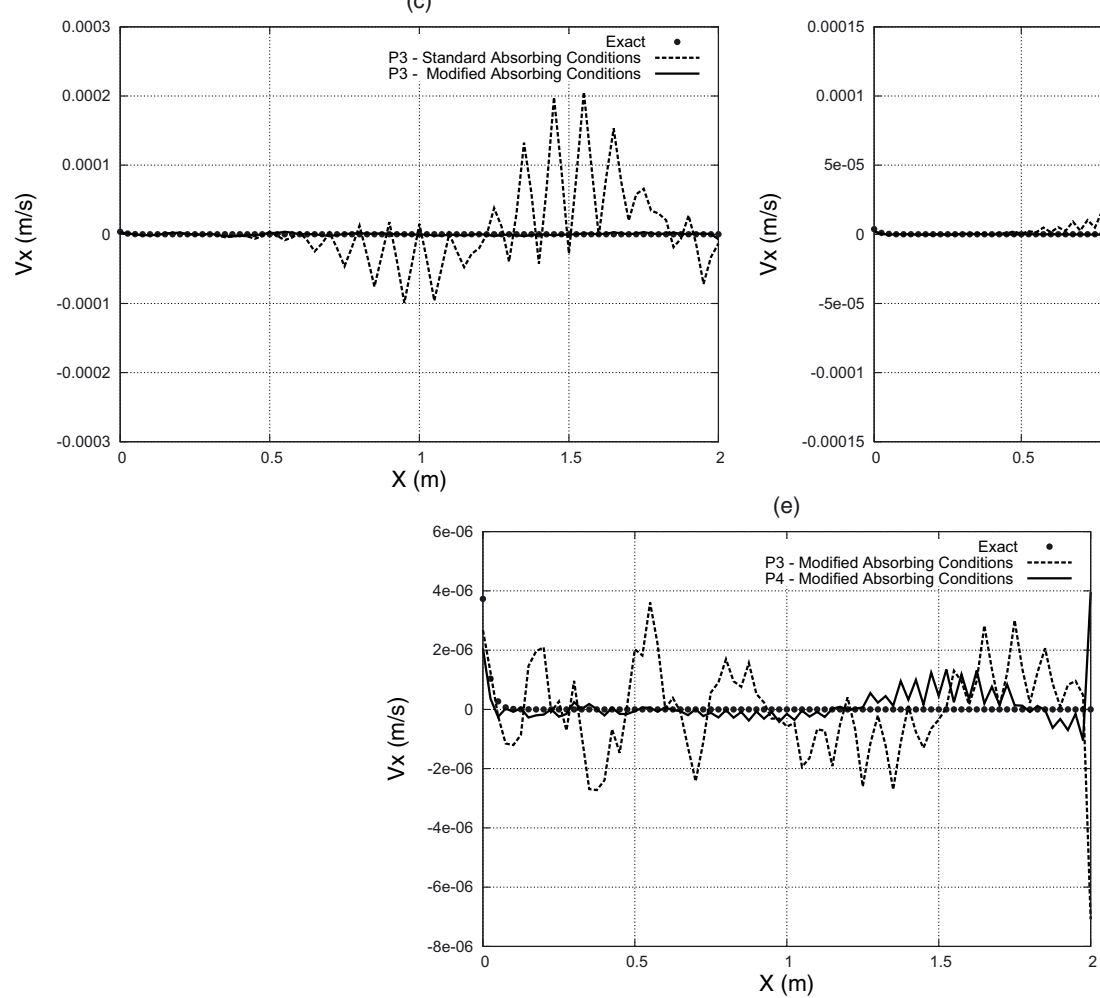

(b)

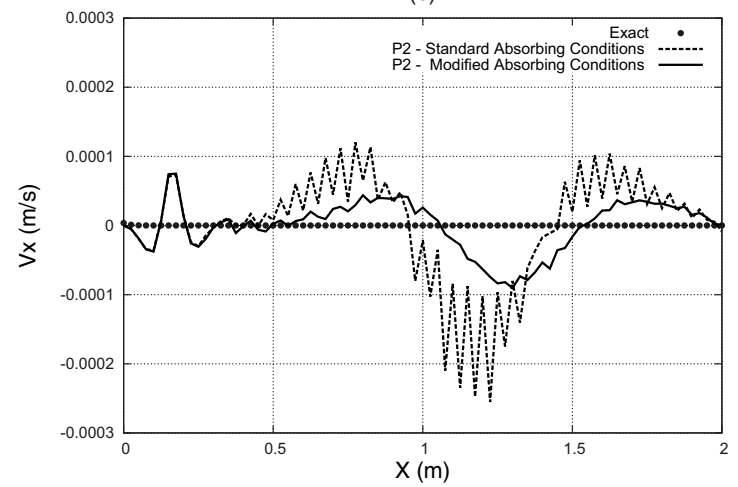

(d)

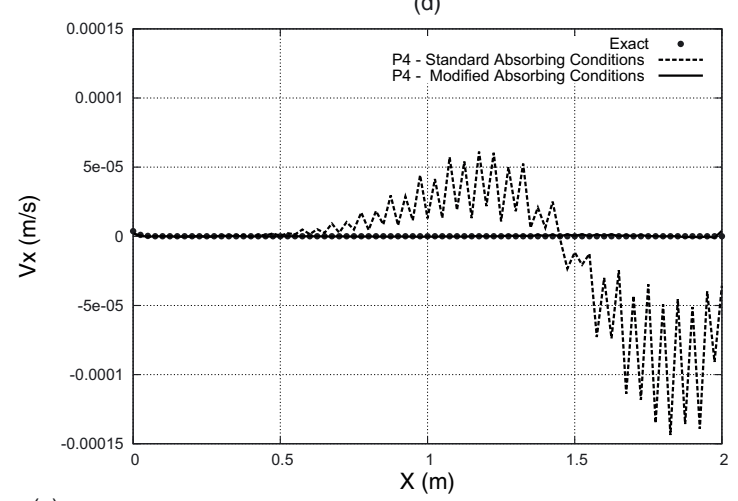

e) 


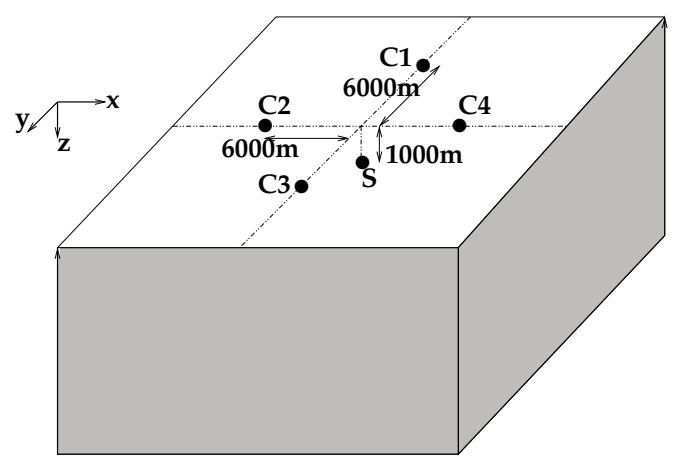

Figure 7. Explosive source in a half space. Position of the source S and the four surface stations.

of the source implementation and an additional study of the absorbing boundary conditions. The medium is homogeneous of density $\rho=2800 \mathrm{~kg} / \mathrm{m}^{3}$ and P- and S-waves velocities are respectively $v_{p}=6400 \mathrm{~m} / \mathrm{s}$ and $v_{s}=3700 \mathrm{~m} / \mathrm{s}$. We apply a free surface condition on the upper boundary of the domain and absorbing conditions on the other boundaries. The source is located at the center of the domain at $1000 \mathrm{~m}$ depth (at the coordinates $(0 ; 0 ; 1000))$ and four surface stations are placed at $6000 \mathrm{~m}$ from the epicenter, symmetrically on the $x$ - and $y$-axis as described in Figure 7. The source signal is a Ricker wavelet whose moment-rate is given by

$$
s(t)=\frac{3 \cdot 10^{16}}{\alpha \sqrt{\pi}} \exp \left[-\frac{(t-1)^{2}}{\alpha^{2}}\right]
$$

with $\alpha=0.25$; it corresponds to a central frequency equal to $f_{c}=1.0 \mathrm{~Hz}$ and a maximum frequency of $f_{\max }=3.0 \mathrm{~Hz}$. Thus, the wavelength $L=v_{s} / f_{\max }$ is approximatively equal to $1233 \mathrm{~m}$. The explosive source is introduced as a right hand side on the diagonal components of the stress tensor i.e. for $\sigma_{x x}, \sigma_{y y}$ and $\sigma_{z z}$. In order to compare the results obtained using several methods $\mathcal{P}_{p}$, especially the finite volume method $\left(\mathcal{P}_{0}\right)$, this right hand side writes $s(t) g(x, y, z)$ where $g$ is defined by

$$
g(x, y, z)=\frac{1}{M} \exp \left[-\frac{\left(x-x_{S}\right)^{2}+\left(y-y_{S}\right)^{2}+\left(z-z_{S}\right)^{2}}{h^{2}}\right] \quad \text { with } \quad M=\int_{\Omega} g(x, y, z) \mathrm{d} v,
$$

where $\left(x_{S}, y_{S}, z_{S}\right)$ is the source coordinates. Note that the support of $g$ is taken sufficiently small, compared to the element size, for an accurate approximation of a point source, a right hand side based on a Dirac function $s(t) \delta\left(x_{S}, y_{s}, z_{S}\right)$ being only accurate for high degree approximation based methods. Initial conditions for the system are $\vec{V}=\overrightarrow{0}$ and $\vec{\sigma}=\overrightarrow{0}$ and the solutions are calculated until $t=6.0 \mathrm{~s}$. We also point out that the finite-volume method presented in this study is a very standard one where the unknowns are set constant in the mesh elements and cannot be compared to high-order finite-volume methods, in particular the one presented by Dumbser et al. [27].

The calculation domain is $36 \mathrm{~km} \times 36 \mathrm{~km} \times 12 \mathrm{~km}$. Solutions have been obtained using several uniform tetraedral meshes obtained, as previously, by dividing the domain in cubic cells of edge $h$ which are split in six tetraedra. The mesh spacing $h$, i.e. here the smallest edge of the mesh, can be expressed as a function of the wavelength $L$ as summarized in Table 3 .

Calculations have been performed on 64 processors using message passing interface (MPI), whatever the mesh spacing, for a better comparison of the CPU times between the different methods. The characteristics of the different meshes are also listed in Table 3.

For this test, we have proposed some criteria in order to estimate the accuracy of the solution. Firstly, we check two particular properties, consequences of an explosive source, which are (i) $V_{x}=0$ at stations $\mathrm{C}_{1}$ and $\mathrm{C}_{3}$ (on $y$-axis) and $V_{y}=0$ at stations $\mathrm{C}_{2}$ and $\mathrm{C}_{4}$ (on $x$-axis) and (ii) the evolution at the surface of the vertical 
TABLE 3. Correspondance between the mesh spacing $h$ and the wavelength $L$ of the source function. Characteristics of the different meshes $\left(N_{T}\right.$ total number of tetraedra, $N_{T} /$ proc., number of tetraedra for each processor).

\begin{tabular}{cccccccc}
\hline$h(\mathrm{~m})$ & 500 & 400 & 300 & 200 & 150 & 100 & 75 \\
\hline$\simeq L / n$ & $L / 2.5$ & $L / 3$ & $L / 4$ & $L / 6$ & $L / 8$ & $L / 12$ & $L / 16$ \\
$N_{T}$ & 746496 & 1458000 & 3456000 & 11664000 & 27648000 & 93312000 & 221184000 \\
$N_{T}$ proc. & 11664 & 22781 & 54000 & 182250 & 432000 & 1458000 & 3456000 \\
\hline
\end{tabular}

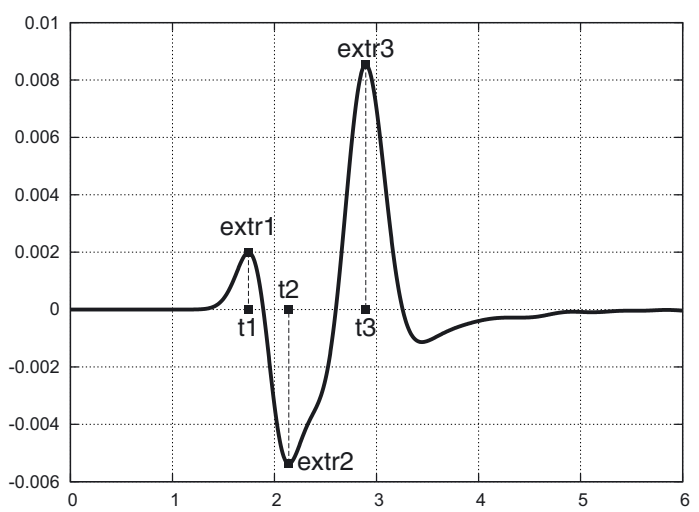

FiguRE 8 . Velocity $V_{z}(\mathrm{~m} / \mathrm{s})$ as a function of time $(\mathrm{s})$; reference solution at station $\mathrm{C}_{1}$. Extrema and corresponding times for the evaluation of errors $\mathrm{err}_{2}$ and $\mathrm{err}_{3}$.

velocity component $V_{z}$ only depends on the distance to the epicenter, thus the $V_{z}$ profiles obtained at the four stations are superimpose. Moreover, for a validation of the source introduction, the amplitudes and the arrival times of the profiles are compared to a reference solution computed at these four stations (and not everywhere in the computational domain) using the Discrete Wave Number method [12]. Then, three different errors are defined. First, $\operatorname{err}_{1}$ is concerned with the components which should ideally be equal to zero and expresses

$$
\operatorname{err}_{1}=\max _{t \in[0 ; ; 6 .]}\left[\left|V_{x}\right|_{C_{1}},\left|V_{y}\right|_{C_{2}},\left|V_{x}\right|_{C_{3}},\left|V_{y}\right|_{C_{4}}\right] .
$$

The two other errors depend on the values at the extrema, as illustrated in Figure 8. For each non null velocity profile (for instance, here, $V_{z}$ for the reference solution at station $\mathrm{C}_{1}$ ), we identify three extrema and note their values $\left(V_{z}\left(\right.\right.$ extr $\left.\left._{i}\right), i=1,2,3\right)$ and the corresponding times $\left(t_{i}, i=1,2,3\right)$. From these data, we define a relative error, in the amplitude or in time, between two profiles (for instance profiles at stations $C_{j}$ and $C_{k}$ ), for a given

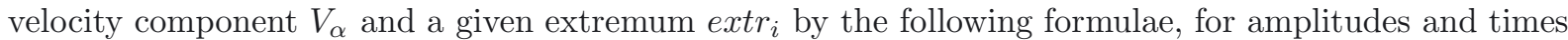

$$
\operatorname{err}_{\mathrm{rel}}\left(V_{\alpha}, C_{j} / C_{k}, e x t r_{i}\right)_{\mathrm{ampl}}=\left|\frac{V_{\alpha}\left(e x t r_{i}\right)_{C_{j}}-V_{\alpha}\left(e x t r_{i}\right)_{C_{k}}}{V_{\alpha}\left(e x t r_{i}\right)_{\mathrm{ref}}}\right|, \quad \operatorname{err}_{\mathrm{rel}}\left(V_{\alpha}, C_{j} / C_{k}, e x t r_{i}\right)_{t}=\left|\frac{t_{i / C_{j}}-t_{i / C_{k}}}{t_{i / r e f}}\right|,
$$

where the subscript $V_{\text {ref }}$ stands for the corresponding value of the reference solution. Thus, error err 2 measures the asymmetries of the computed solution (i.e. the mean maximum error of the amplitude and the travel time between solutions computed at two stations) of the vertical velocity component $V_{z}$ which writes

$$
\operatorname{err}_{2 / a m p l}=\frac{1}{3} \sum_{i=1}^{3} \max _{1 \leq j, k \leq 4} \operatorname{err}_{\mathrm{rel}}\left(V_{z}, C_{j} / C_{k}, e x t r_{i}\right)_{\mathrm{ampl}}, \quad \operatorname{err}_{2 / t}=\frac{1}{3} \sum_{i=1}^{3} \max _{1 \leq j, k \leq 4} \operatorname{err}_{\mathrm{rel}}\left(V_{z}, C_{j} / C_{k}, \operatorname{extr}_{i}\right)_{t} .
$$


TABLE 4. CPU times of the calculations for the $\mathcal{P}_{p}$ methods and the different meshes on 64 processors.

\begin{tabular}{|c|c|c|c|c|c|c|c|}
\hline & & & & CPU time & & & \\
\hline $\mathcal{P}_{p} / \mathrm{h}$ & 500 & 400 & 300 & 200 & 150 & 100 & 75 \\
\hline $\mathcal{P}_{0}$ & - & - & $26 \mathrm{~s}$ & $2 \min 10 \mathrm{~s}$ & $6 \min 18 \mathrm{~s}$ & $29 \min 34 \mathrm{~s}$ & $1 \mathrm{~h} 30 \mathrm{~min}$ \\
\hline $\mathcal{P}_{1}$ & - & - & $5 \min 44 \mathrm{~s}$ & $24 \min 56 \mathrm{~s}$ & $1 \mathrm{~h} 13 \mathrm{~min}$ & $6 \mathrm{~h} 7 \mathrm{~min}$ & $21 \mathrm{~h} 51 \mathrm{~min}$ \\
\hline $\mathcal{P}_{2}$ & - & $10 \min 10 \mathrm{~s}$ & $27 \min 19 \mathrm{~s}$ & $2 \mathrm{~h} 15 \mathrm{~min}$ & $7 \mathrm{~h}$ & - & - \\
\hline $\mathcal{P}_{3}$ & - & $26 \min 26 \mathrm{~s}$ & $1 \mathrm{~h} 14 \mathrm{~min}$ & $6 \mathrm{~h}$ & - & - & - \\
\hline $\mathcal{P}_{4}$ & $26 \min 33 \mathrm{~s}$ & $1 \mathrm{~h} 11 \mathrm{~min}$ & $3 \mathrm{~h} 20 \mathrm{~min}$ & - & - & - & - \\
\hline
\end{tabular}

Finally, $\mathrm{err}_{3}$ is the mean error between the computed and the reference solutions at all stations, for all non null velocity components and for all extrema. This error writes, for instance for amplitudes

$$
\begin{aligned}
\operatorname{err}_{3 / \text { ampl }}= & \frac{1}{24} \sum_{i=1}^{3}\left[\sum_{j \in\{2,4\}} \operatorname{err}_{\mathrm{rel}}\left(V_{x}, C_{j} / C_{j / r e f}, \text { extr }_{i}\right)_{\mathrm{ampl}}\right. \\
& \left.+\sum_{j \in\{1,3\}} \operatorname{err}_{\mathrm{rel}}\left(V_{y}, C_{j} / C_{j / r e f}, \text { extr }_{i}\right)_{\mathrm{ampl}}+\sum_{j=1}^{4} \operatorname{err}_{\mathrm{rel}}\left(V_{z}, C_{j} / C_{j / r e f}, \text { extr }_{i}\right)\right],
\end{aligned}
$$

an equivalent definition being applied to define $\mathrm{err}_{3 / t}$.

Calculations have been done using methods based on different degrees of interpolation $(0 \leq p \leq 4)$ in combination with the meshes defined in Table 3 in order to study the convergence and CPU time corresponding to the three types of errors; the results of these calculations are gathered in Figure 9 and the CPU times of each calculation are given in Table 4 . Note that, as previously, $\mathcal{P}_{p}$ refers to a method based on polynomials of degree $p$ and the standard leap-frog time method. This first series of results is obtained using the standard absorbing boundary condition.

The first line of the figure presents the values of $\operatorname{err}_{1}$ as a function of the mesh spacing $h$ (Fig. 9a) and by respect to the corresponding CPU time (Fig. 9b). When examining these results, we notice (Fig. 9a) that $\operatorname{err}_{1}$ corresponding to $\mathcal{P}_{0}$ method remains nearly constant, whatever the mesh spacing, at about $10 \%$ of the maximum value of the velocity. Results are clearly improved by the use of higher degree methods since, in comparison with the $\mathcal{P}_{0}$ results, the value of $\operatorname{err}_{1}$ of the $\mathcal{P}_{2}$ method is reduced by a factor $10^{3}$. Moreover, higher degree methods $\mathcal{P}_{3}$ and $\mathcal{P}_{4}$ are also more efficient since a given error level of accuracy is obtained for lower CPU times.

Now, we study the second line of figures corresponding to $\mathrm{err}_{2}$, which is a relative error traducing the asymmetry of the $V_{z}$ profiles by respect to $h$ (Fig. 9c) and the CPU time (Fig. 9d). As previously, we notice that the results of the $\mathcal{P}_{0}$ method are not accurate enough even using the finest meshes. The improvement on the symmetry is obvious when using the other methods, especially the methods with $p \geq 2$ resulting in $\mathrm{err}_{2}$ values lower than $0.1 \%$. As previously for $\operatorname{err}_{1}, \mathcal{P}_{3}$ and $\mathcal{P}_{4}$ are the two most efficient methods.

Finally, we analyze the results obtained for $\operatorname{err}_{3}$ (Figs. 9e and 9f) which is a relative error between the computed and the reference solutions at the extrema. As previously, the lowest error levels correspond to the $\mathcal{P}_{2}$ to $\mathcal{P}_{4}$ methods whereas the $\mathcal{P}_{0}$ method produces relative errors in the order of $10 \%$ and this independently on $h$. The other methods allow errors lower than $5 \%$ when using the finest meshes. Note that, for this last error, if the improvement between $\mathcal{P}_{0}$ and $\mathcal{P}_{1}$ is obvious, it is more limited for the highest degree methods. Furthermore, these methods lead to comparable levels of error. This fact is also visible when $\mathrm{err}_{3}$ is plotted as a function of the CPU time (Fig. 9f). The reduction of the error levels for the highest values of $p$ is not sufficient to compensate their overcost; thus, for this last criterion, the $P_{1}$ method appears to be most efficient.

We also present, in Figure 10, the errors $\operatorname{err}_{2 / t}$ (Fig. 10a) and $\operatorname{err}_{3 / t}$ (Fig. 10b) on arrival times as a function of the mesh spacing. This error is plotted only for $\mathcal{P}_{0}$ and $\mathcal{P}_{1}$ methods because it is equal to zero for $2 \leq p \leq 4$, 
(a)

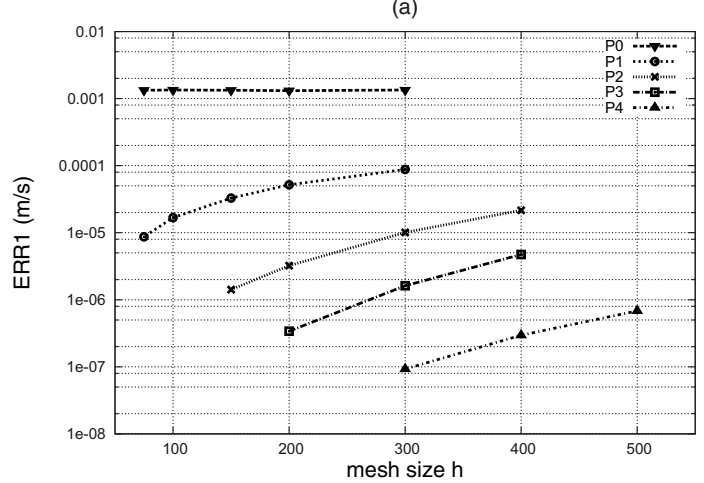

(c)

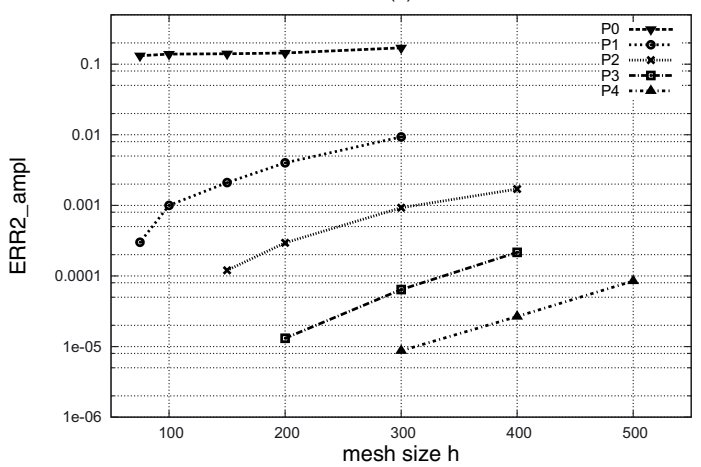

(e)

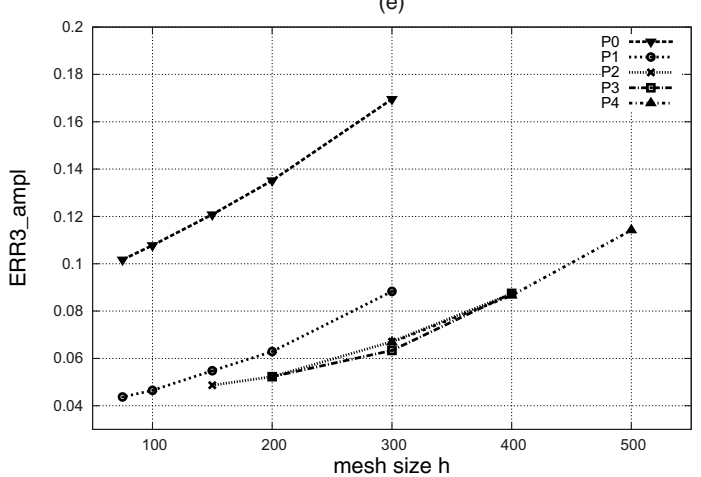

(b)

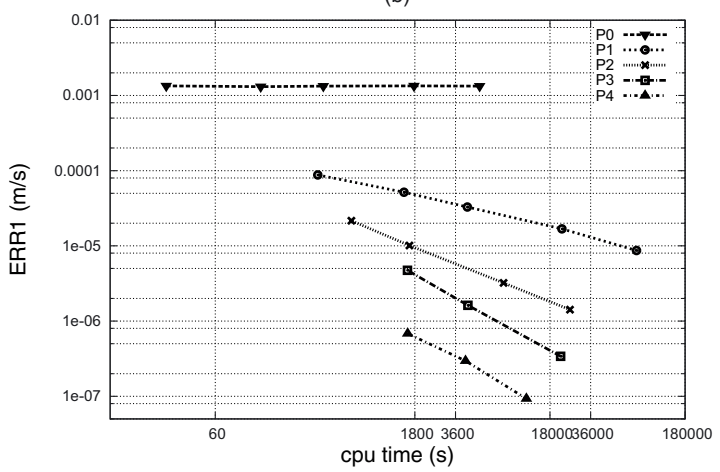

(d)

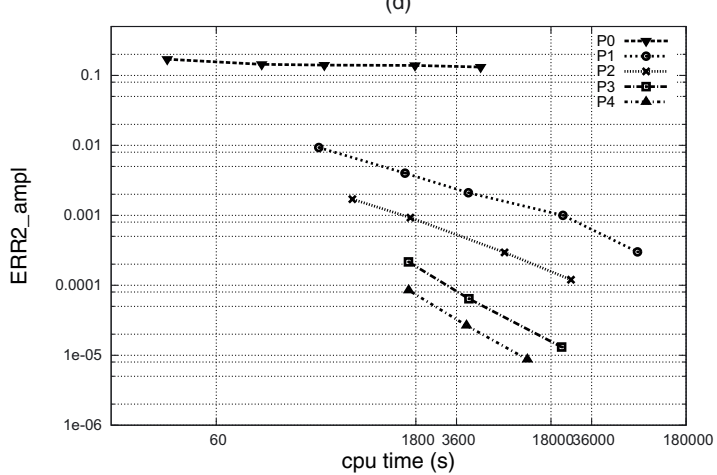

(f)

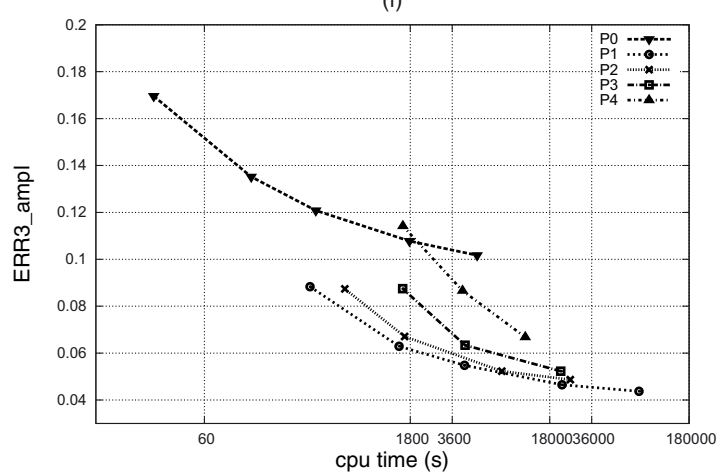

Figure 9. Convergence and CPU time for errors on amplitude, err 1 (a and b), $\operatorname{err}_{2}$ (c and d) and $\operatorname{err}_{3}$ (e and f). Explosive source in a half space, solutions at $t=6$. s, uniform meshes, computations on 64 processors.

whatever the value of $h$. Even for fine meshes, the arrival times are not exactly the same for $p=0$ and $p=1$. On Figure 10b, which corresponds to $\operatorname{err}_{3 / t}$, we clearly distinguish the $\mathcal{P}_{0}$ method for which the relative error is greater than $1 \%$. This error is reduced using the $\mathcal{P}_{1}$ method and very low for $p \geq 2$. In first conclusion of this study, we have to distinguish results obtained with the $\mathcal{P}_{0}$ method from those of the other methods. First of all, the $\mathcal{P}_{0}$ method leads to solutions that do not verify the symmetry condition, for amplitudes and arrival times, and the use of fine meshes do not allow improving these results. Better solutions are obtained with the $\mathcal{P}_{1}$ method but the use of higher-degree space interpolations clearly helps improving the different types of errors. 
(a)

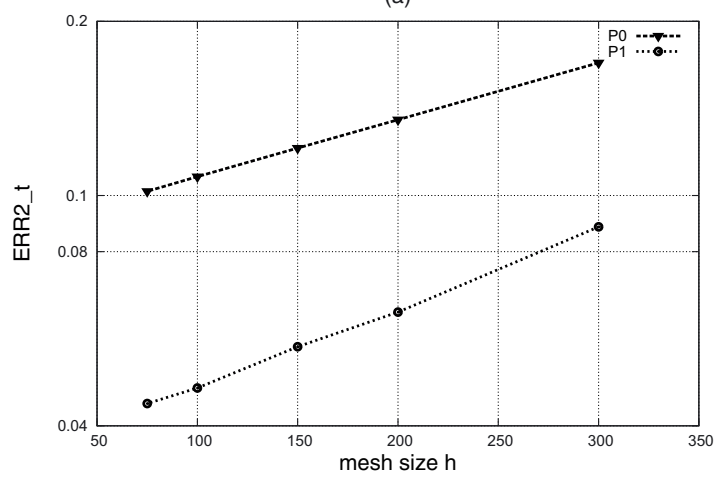

(b)

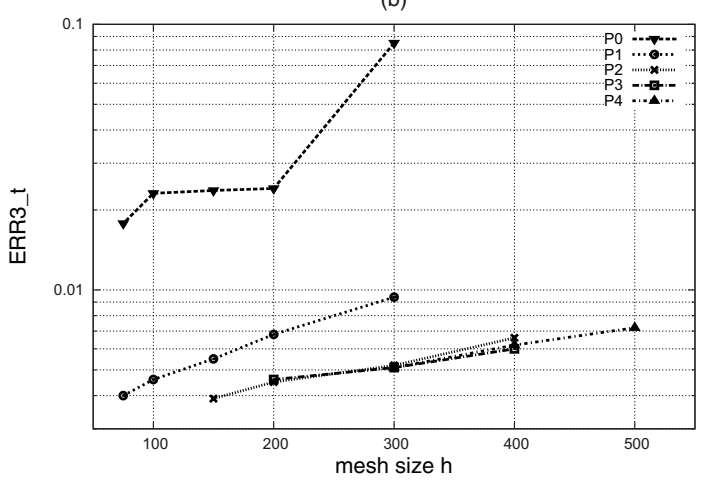

Figure 10. Convergence on arrival times, $\operatorname{err}_{2 / t}$ (a) and $\operatorname{err}_{3 / t}$ (b). Explosive source in a half space, solutions at $t=6 \mathrm{~s}$, uniform meshes, computations on 64 processors.
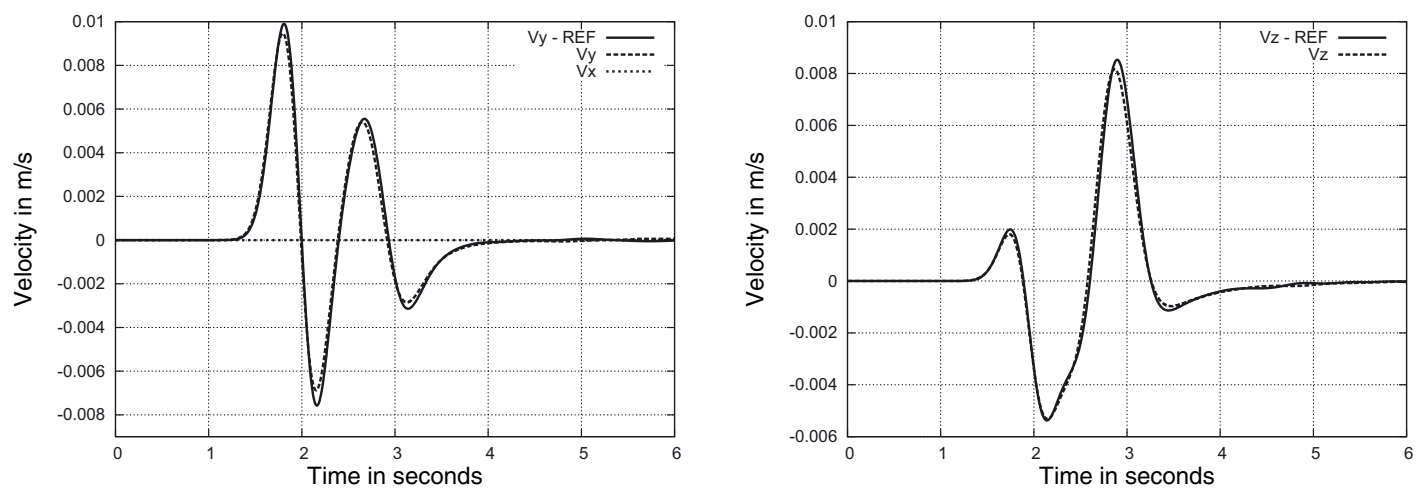

Figure 11. Comparison between computed solution and reference at station $\mathrm{C}_{1} \cdot V_{x}$ and $V_{y}$ (left picture) and $V_{z}$ (right picture). Computed solution corresponds to $P_{2}$ method for a mesh spacing equal to $200 \mathrm{~m}$.

When combining the different criteria on the solution, the $\mathcal{P}_{2}$ method seems to be a good compromise between accuracy and efficiency. We notice that the error $\mathrm{err}_{3}$ on amplitude between our solutions and the reference seems to converge towards a value equal to $4-5 \%$. It would be interesting to compare these results with those corresponding to a source introduced by a Dirac function, perhaps more suitable with high-order methods.

The results obtained, at station $\mathrm{C}_{1}$, with the method $\mathcal{P}_{2}$ and a mesh spacing $h=200 \mathrm{~m}$ are presented in Figure 11. They are also compared to the reference solution. This constitutes a validation of the source implementation and of the absorbing boundary conditions since no spurious reflections from the boundaries appear in the profiles.

In a second step, we realize some additional simulations in order to compare the two types of absorbing boundary conditions ( $\alpha=1$ and 2), already studied in the previous test case. For this study, we choose the $\mathcal{P}_{4}$ method and set the mesh size to $h=300 \mathrm{~m}$. Solutions are calculated until time $10.0 \mathrm{~s}$ using two computational domains: the initial one, which is $36 \mathrm{~km} \times 36 \mathrm{~km} \times 12 \mathrm{~km}$, and a reduced one, $24 \mathrm{~km} \times 24 \mathrm{~km} \times 12 \mathrm{~km}$. For this second domain, the boundary is $6 \mathrm{~km}$ closer to the stations than for the initial one. We compare in Figure 12 the solutions for velocities $V_{x}$ and $V_{z}$ at station $\mathrm{C}_{1}$, on both domains and using both types of boundary conditions. When we examine the solutions for $V_{x}$ (Figs. 12a and 12b), we notice that reducing the domain produces some reflections, especially for times greater than $6.0 \mathrm{~s}$. The effect of the modified absorbing condition is noticeable 
(a)

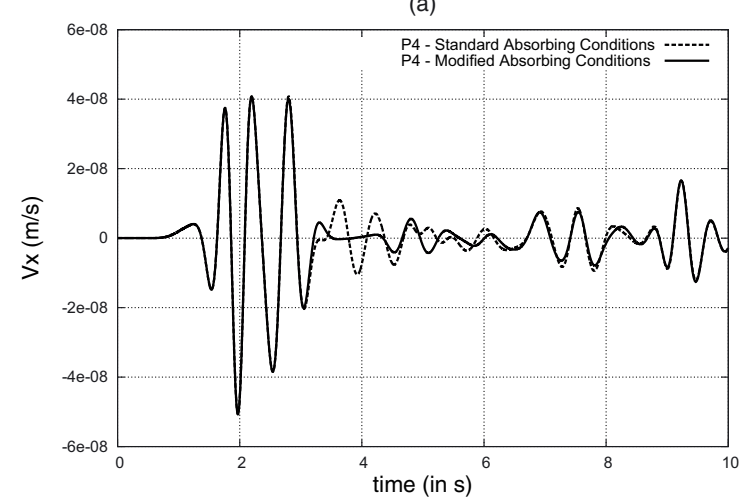

(c)

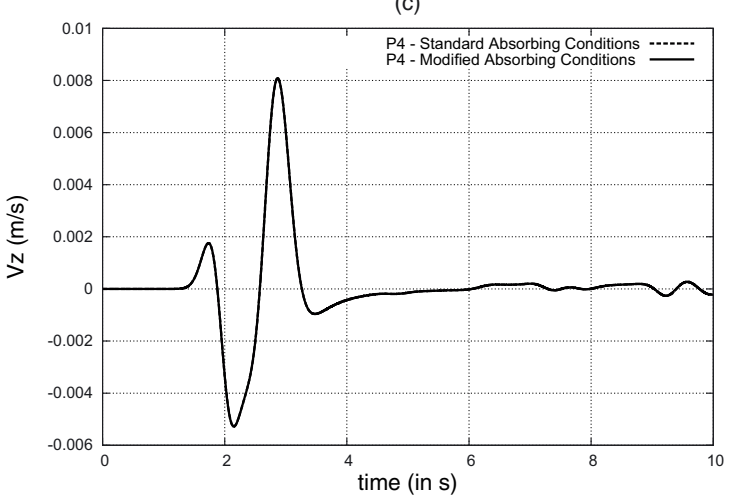

(b)

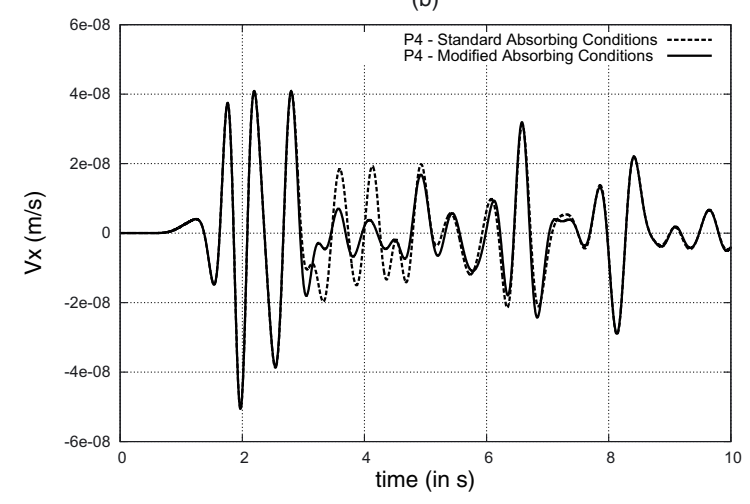

(d)

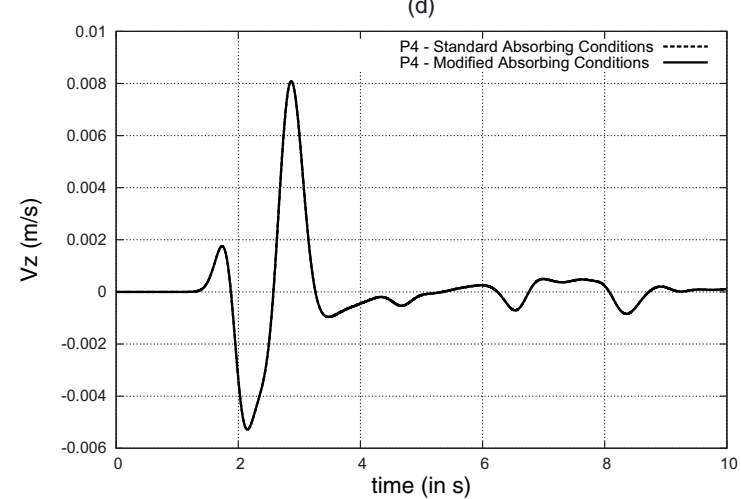

Figure 12. Comparison of the solutions of the $\mathcal{P}_{4}$ method with $h=300 \mathrm{~m}$, using the initial and a reduced domain. $V_{x}$ at station $\mathrm{C}_{1}$, comparison between absorbing boundary conditions using the large domain (a) and the reduced domain (b). Same results for $V_{z}$ at station $\mathrm{C}_{1}$ for initial (c) and reduced domain (d).

on both figures, but only in the time interval $[3 . ; 5$.$] s. Comparing now the results for V_{z}$, Figures $12 \mathrm{c}$ and $12 \mathrm{~d}$, we find no difference due to the boundary conditions. Reflections are clearly visible and only explained by the distance to the boundary. If the modified absorbing condition allowed a clear improvement in the previous case, for a wave propagating perpendicular to the boundary, it does not reduce the reflections in this case, in presence of grazing waves, especially for the reduced domain.

\section{Conclusion}

We proposed a discontinuous Galerkin finite element method to solve the first-order hyperbolic system of the elastodynamic equations, written in velocity-stress formulation. This method is applied to tetraedral meshes allowing an accurate approximation of the medium or mesh refinement in particular areas of the domain. The spatial approximation is based on high-degree polynomials defined locally on each element of the mesh. The scheme combines centered fluxes and a leap-frog scheme in time which leads to a non diffusive method. We also detailed a simple absorbing boundary condition, derived from an upwind scheme. In this paper, the properties of the method have been analysed in detail. First, we have proved that this explicit scheme is stable under a condition on the time-step resulting from a discrete energy which is preserved in domains with free surfaces and decreasing in presence of absorbing boundary conditions. Moreover, the convergence of the method for the semi-discrete and the fully discrete schemes has been studied. More precisely, for solutions regular enough, the 
scheme is convergent with an order $\mathcal{O}\left(\Delta t^{N}+h^{k}\right)$ for domains with only free surface boundary conditions, or $\mathcal{O}\left(\Delta t^{\min (\alpha, 2)}+h^{k}\right)$ when the absorbing boundary conditions are included in the scheme, where $k$ is the degree of the polynomial approximation, $N$ is the order of the leap-frog scheme and $\alpha \in\{1,2,3,4\}$ is defined by (3.28).

Three-dimensional numerical results have been obtained with a parallel implementation of the DG method. The first one refers to the propagation of an eigenmode in a cubic cavity for which an exact solution is known and a numerical study of the convergence has been realised for series of uniform meshes, comparing the standard and the fourth-order leap-frog time scheme. In a second application, we study the propagation of a pulse which allows comparing two types of boundary conditions. Finally, The last test-case concerns the propagation of the waves due to an explosive source in a half-space which enables a validation of the source implementation and the absorbing boundary conditions. The main conclusions of this study are the accuracy and the efficiency of the method particularly for higher-degree polynomial interpolations. These results highlight the gain obtained with these methods, especially when compared to the standard finite volumes method $\left(\mathcal{P}_{0}\right)$ for which mesh refinement did not enable improving both errors on amplitudes and arrival time. The good compromise between accuracy and CPU time seems obtained using the P2 method (based on polynomials of degree 2). Moreover, the free surface condition is accurately approximated. These results are encouraging and could be developed in several interesting directions. First, the treatment at absorbing boundaries could be improved using more accurate boundary conditions. Many techniques have been proposed to reduce spurious reflections, including additional absorbing layers which may be costly in 3D. Then, high-order non reflecting boundary conditions [34] or an implicit treatment of our simple absorbing condition could be interesting ways of improvement, especially for grazing waves.

Finally, we are interested in simulations of more realistic three dimensional problems including topography and realistic media.

Acknowledgements. The authors are very grateful to anonymous reviewers for their critical remarks that have contributed to improve the quality of the paper. This work was partially supported by the European Research Council ERC Starting Grant 2009, project 239983-NuSiKiMo.

\section{REFERENCES}

[1] S. Adjerid, K. Devine, J. Flaherty and L. Krivodonova, A posteriori error estimation for discontinuous Galerkin solutions of hyperbolic problems. Comput. Methods Appl. Mech. Engrg. 191 (2002) 1097-1112.

[2] C. Agut, J. Diaz and A. Ezziani, High-order schemes combining the modified equation approach and discontinuous Galerkin approximations for the wave equation. Commun. Comput. Phys. 11 (2012) 691-708.

[3] M. Ainsworth, Dispersive and dissipative behaviour of high order discontinuous Galerkin finite element methods. J. Comput. Phys. 19 (2004) 106-130.

[4] M. Ainsworth, P. Monk and W. Muniz, Dispersive and dissipative properties of discontinuous Galerkin finite element methods for the second-order wave equation. J. Sci. Comput. 27 (2006) 5-40.

[5] P.F. Antonietti, I. Mazzieri, A. Quarteroni and F. Rapetti, Non-conforming high order approximations of the elastodynamics equation. Comput. Methods Appl. Mech. Engrg. 209-212 (2012) 212-238.

[6] S. Aoi and H. Fujiwara, 3D Finite-difference method using discontinuous grids. Bull. Seism. Soc. Am. 89 (1999) $918-930$.

[7] Z. Alterman and F.C. Karal, Propagation of elastic waves in layered media by finite-difference methods. Bull. Seism. Soc. Am. 58 (1968) 367-398.

[8] H. Bao, J. Bielak, O. Ghattas, L. Kallivokas, D.R. O'Hallaron, J.R. Schewchuk and J. Xu, Large-scale simulation of elastic wave propagation in heterogeneous media on parallel computers. Comput. Methods Appl. Mech. Engrg. 152 (1998) 85-102.

[9] A. Bayliss, K.E. Jordan, B.J. LeMesurier and E. Turkel, A fourth-order accurate finite-difference scheme for the computation of elastic waves. Bull. Seism. Soc. Am. 76 (1986) 1115-1132.

[10] M. Benjemaa, Étude et simulation numérique de la rupture dynamique des séismes par des méthodes d'éléments finis discontinus. Ph.D. thesis, Nice-Sophia Antipolis University (2007).

[11] M. Benjemaa, S. Piperno and N. Glinsky-Olivier, Étude de stabilité d'un schéma volumes finis pour les équations de l'élastodynamique en maillages non structurés, INRIA report 5817 (2006).

[12] M. Bouchon and K. Aki, Discrete wave-number representation of seismic-source wave fields. Bull. Seism. Soc. Am. 67 (1977) $259-277$.

[13] T. Bui-Thanh and O. Ghattas, Analysis of an hp-nonconforming discontinuous Galerkin spectral element method for wave propagation. SIAM J. Numer. Anal. 50 (2012) 1801-1826. 
[14] E. Chaljub, Y. Capdeville and J.P. Vilotte, Solving elastodynamics in a fluid-solid heterogeneous sphere: a parallel spectral element approximation on non-conforming grids. J. Comput. Phys. 187 (2003) 457-491.

[15] Y. Cheng and C.-W. Shu, Superconvergence and time evolution of discontinuous Galerkin finite element solutions. J. Comput. Phys. 227 (2008) 9612-9627.

[16] N. Chevaugeon, K. Hillewaert, X. Gallez, P. Ploumhans and J.-F. Remacle, Optimal numerical parameterization of discontinuous Galerkin method applied to wave propagation problems. J. Comput. Phys. 223 (2007) 188-207.

[17] P. Ciarlet, The finite element method for elliptic problems. North Holland-Elsevier science publishers, Amsterdam, New York, Oxford (1978).

[18] B. Cockburn and C.W. Shu, TVB Runge-Kutta local projection discontinuous Galerkin finite element method for conservation laws II: general framework. Math. Comput. 52 (1989) 411-435.

[19] B. Cockburn and C.W. Shu, The Runge-Kutta discontinuous Galerkin method for conservation laws V: multidimensional systems. J. Comput. Phys. 141 (1998) 199-224.

[20] B. Cockburn, S.Y. Lin and C.W. Shu, TVB Runge-Kutta local projection discontinuous Galerkin finite element method for conservation laws III: one dimensional systems. J. Comput. Phys. 84 (1989) 90-113.

[21] B. Cockburn, S. Hou and C.W. Shu, The Runge-Kutta local projection discontinuous Galerkin finite element method for conservation laws IV: the multidimensional case. Math. Comput. 54 (1990) 545-581.

[22] B. Cockburn, G.E. Karnadiakis and C.W. Shu, Discontinuous Galerkin Methods: Theory, Computation and Application. Lect. Notes Comput. Sci. Engrg. Springer, Berlin (2000).

[23] J.D. De Basabe, M.K. Sen and M. Wheeler, The interior penalty discontinuous Galerkin method for elastic wave propagation: grid dispersion. Geophys. J. Int. 175 (2008) 83-93.

[24] L. Demkowicz and J. Kurtz, Projection-based interpolation and automatic hp-adaptivity for finite element discretizations of elliptic and Maxwell problems. ESAIM: Proc. 21 (2007) 1-15.

[25] S. Delcourte, L. Fezoui and N. Glinsky-Olivier, A high-order Discontinuous Galerkin method for the seismic wave propagation. ESAIM Proc. 27 (2009) 70-89.

[26] M. Dumbser and M. Käser, An arbitrary high-order Discontinuous Galerkin method for elastic waves on unstructured meshes II: the three-dimensional isotropic case. Geophys. J. Int. 167 (2006) 319-336.

[27] M. Dumbser, M. Käser and J. de la Puente, Arbitrary high order finite volume schemes for seismic wave propagation on unstructured meshes in 2D and 3D. Geophys. J. Int. 171 (2007) 665-694.

[28] V. Etienne, E. Chaljub, J. Virieux and N. Glinsky, An hp-adaptive discontinuous Galerkin finite-element method for 3-D elastic wave modelling. Geophys. J. Int. 183 (2010) 941-962.

[29] H. Fahs, High-order Leap-Frog based discontinuous Galerkin method for the time-domain Maxwell equations on non-conforming simplicial meshes. Numer. Math. Theor. Methods Appl. 2 (2009) 275-300.

[30] L. Fezoui, S. Lanteri, S. Lohrengel and S. Piperno, Convergence and stability of a Discontinuous Galerkin time-domain method for the 3D heterogeneous Maxwell equations on unstructured meshes. ESAIM: M2AN 39 (2005) 1149-1176.

[31] J.S. Hesthaven and T. Warburton, Nodal discontinuous Galerkin methods: algorithms, analysis and applications. Texts Appl. Math. 54 (2008).

[32] F.Q. Hu, M.Y. Hussaini and P. Rasetarina, An analysis of the discontinuous Galerkin method for wave propagation problems. J. Comput. Phys. 151 (1999) 921-946.

[33] M. Galis, P. Moczo and J. Kristek, A 3-D hybrid finite-difference-finite-element viscoelastic modelling of seismic wave motion. Geophys. J. Int. 175 (2008) 153-184.

[34] D. Givoli, High-order local non-reflecting boundary conditions: a review. Wave Motion 39 (2004) 319-326.

[35] N. Glinsky, S. Moto Mpong and S. Delcourte, A high-order discontinuous Galerkin scheme for elastic wave propagation. INRIA report No. 7476 (2010).

[36] C. Johnson and J. Pitkäranta, An analysis of the discontinuous Galerkin method for a scalar hyperbolic equations. Math. Comput. 46 (1986) 1-26.

[37] C. Johnson, U. Nävert and J. Pitkäranta, Finite element methods for linear hyperbolic equations. Comput. Methods Appl. Mech. Engrg. 45 (1984) 285-312,.

[38] M. Käser and M. Dumbser, An arbitrary high-order discontinuous Galerkin method for elastic waves on unstructured meshes I: the two-dimensional isotropic case with external source term. Geophys. J. Int. 162 (2006) 855-877.

[39] M. Käser, M. Dumbser, J. de la Puente and H. Igel, An arbitrary high-order discontinuous Galerkin method for elastic waves on unstructured meshes III: viscoelastic attenuation. Geophys. J. Int. 168 (2007) 224-242.

[40] M. Käser, V. Hermann and J. de la Puente, Quantitative accuracy analysis of the discontinuous Galerkin method for seismic wave propagation. Geophys. J. Int. 173 (2008) 990-999,.

[41] K.R. Kelly, R.W. Ward, S. Treitel and R.M. Alford, Synthetic seismograms: a finite-difference approach. Geophys. 41 (1976) $2-27$.

[42] D. Komatitsch and J.P. Vilotte, The spectral-element method: an efficient tool to simulate the seismic response of 2D and 3D geological structures. Bull. Seism. Soc. Am. 88 (1998) 368-392.

[43] T. Lähivaara and T. Huttunen, A non-uniform basis order for the discontinuous Galerkin method of the acoustic and elastic wave equations. Appl. Numer. Math. 61 (2011) 473-486.

[44] P. Lesaint and P.-A. Raviart, On a finite element method for solving the neutron transport equation, Mathematical Aspects of Finite Element Methods in Partial Differential Equations, edited by C.A. deBoor. Academic Press, New York (1974) 89-123.

[45] A.R. Levander, Fourth-order finite-difference P-SV seismograms. Geophys. 53 (1988) 1425-1436. 
[46] J. Lysmer and L.A. Drake, A finite element method for seismology, in Methods of Computational Physics, edited by B.A. Bolt. Academic Press, New York 11 (1972) 181-216.

[47] R. Madariaga, Dynamics of an expanding circular fault. Bull. Seis. Soc. Am. 66 (1976) 639-666.

[48] K.J. Marfurt, Accuracy of finite-difference and finite-element modelling of the scalar and elastic wave equations. Geophys. 49 (1984) 533-549.

[49] I. Mazzieri and F. Rapetti, Dispersion analysis of triangle-based spectral elements methods for elastic wave propagation. Numer. Algorithms 60 (2012) 631-650.

[50] E.D. Mercerat, J.-P. Vilotte and F. Sanchez-Sesma, Triangular spectral element simulation of 2D elastic wave propagation using unstructured triangular grids. Geophys. J. Int. 166 (2006) 679-698.

[51] P. Moczo, E. Bystrický, J. Kristek, J.M. Carcione and M. Bouchon, Hybrid modeling of P-SV seismic motion at inhomogeneous viscoelastic topographic structures. Bull. Seism. Soc. Am. 87 (1997) 1305-1323.

[52] C. Pelties, J. de la Puente, J.P. Ampuero, G. Brietzke and M. Käser, Three-dimensional dynamic rupture simulations with a high-order discontinuous Galerkin method on unstructured tetrahedral meshes. J. Geophys. Res. 117 (2012) B02309.

[53] F. Peyrusse, N. Glinsky, C. Gélis and S. Lanteri, A nodal discontinuous Galerkin method for site effects assessment in viscoelastic media. Verification and validation in the Nice basin. Geophys. J. Int. (2014) 315-334.

[54] A. Pitarka, 3D elastic finite-difference modeling of seismic motion using staggered-grids with nonuniform spacing. Bull. Seism. Soc. Am. 89 (1999) 85-106.

[55] W. Reed and T. Hill, Triangular mesh methods for the neutron transport equation. Technical Report LA-UR-73-479, Los Alamos Scientific Laboratory, Los Alamos, NM (1973).

[56] C. Scheid and S. Lanteri, Convergence of a Discontinuous Galerkin scheme for the mixed time domain Maxwell's equations in dispersive media. INRIA Report 7634 (2011).

[57] E.H. Saenger, N. Gold and S.A. Shapiro, Modeling the propagation of elastic waves using a modified finite-difference grid. Wave Motion 31 (2000) 77-92.

[58] S. Sherwin, Dispersion analysis of the continuous and discontinuous Galerkin formulation. Lect. Notes Comput Sci. Engrg. 11 (2000) 425-432.

[59] E. Süli, C. Schwab and P. Houston, hp-DGFEM for partial differential equations with non-negative characteristics form, in Discontinuous Galerkin Methods Theory. Computation and Applications, edited by B. Cockburn, G.E. Karnadiakis and C.W. Shu. In vol. 11 of Lect. Notes Comput. Sci. Eng. Springer, Berlin (2000) 221-230.

[60] J. Tago, V.M. Cruz-Atienza, J. Virieux, V. Etienne and F.J. Sanchez-Sesma, A 3D hp-adaptive discontinuous Galerkin method for modelling earthquake dynamics. J. Geophys. Research: Solid Earth 117 (2012).

[61] J. Virieux, P-SV wave propagation in heterogeneous media, velocity-stress finite difference method. Geophys. 51 (1986) 889-901.

[62] J.L. Young, High-order, leapfrog methodology for the temporally dependent Maxwell's equations. Radio Sci. 36 (2001) 9-17.

[63] L.C. Wilcox, G. Stadler, C. Burstedde and O. Ghattas, A high-order discontinuous Galerkin method for wave propagation through coupled elastic-acoustic media. J. Comput. Phys. 229 (2010) 9373-9396.

[64] X. Zhong and C.-W. Shu, Numerical resolution of discontinuous Galerkin methods for time dependent wave equations. Comput. Methods Appl. Mech. Engrg. 200 (2011) 2814-2827. 